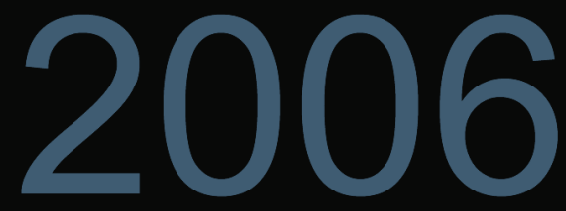

\title{
Nevada Test Site Annual Illness and Injury Surveillance Report
}

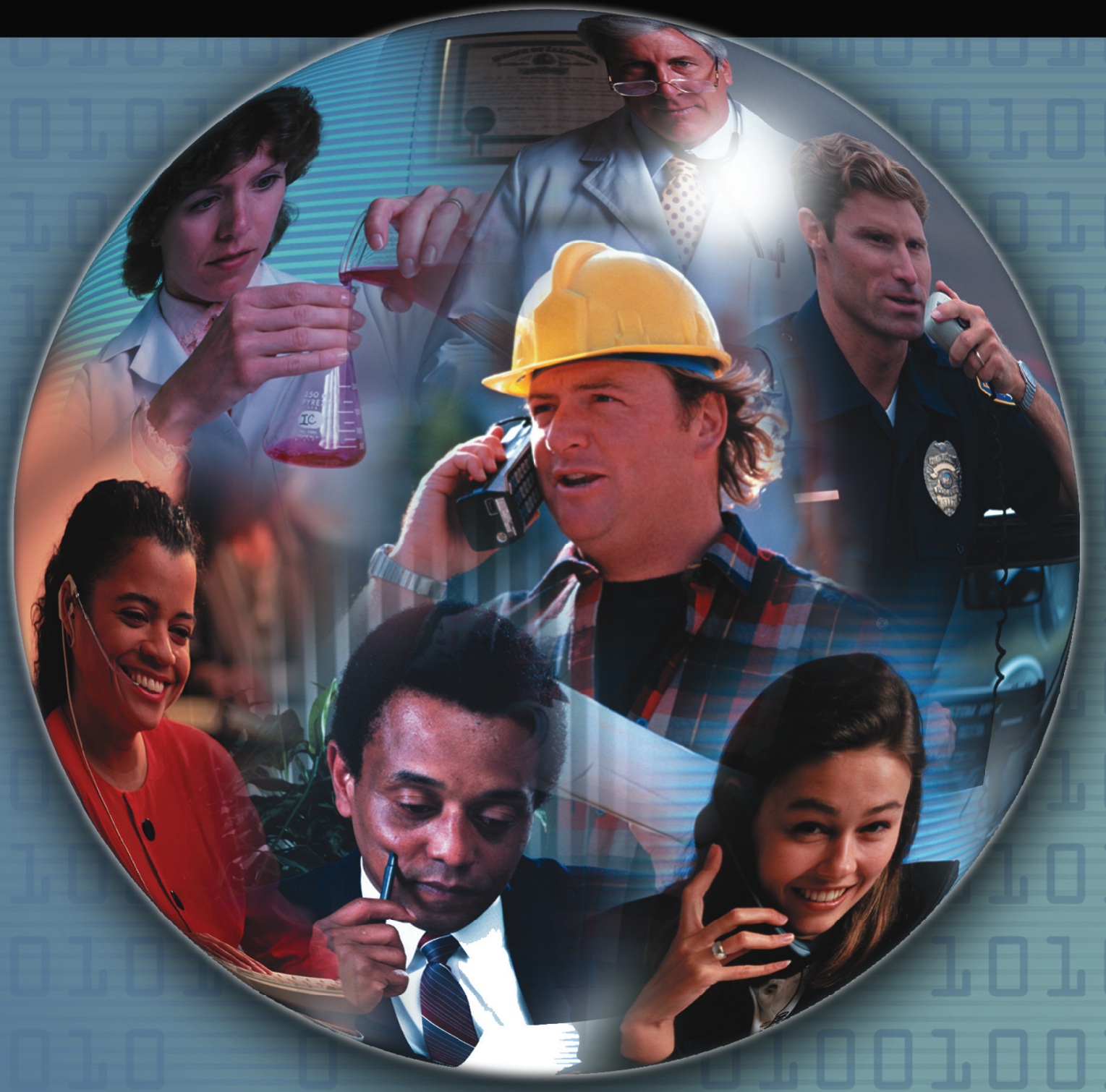




\title{
Nevada Test Site \\ 2006 Illness and Injury Surveillance Report
}

Questions or comments about this report or the Illness and Injury Surveillance Program (IISP) may be directed to:

E-mail:

Dr. Cliff Strader at cliff.strader@hq.doe.gov or Dr. Bonnie Richter at bonnie.richter@hq.doe.gov

or direct letters to:

\author{
Mail Stop HS-13 / 270CC \\ U.S. Department of Energy \\ 1000 Independence Avenue, S.W. \\ Washington, DC 20585-0270
}

Additional information about the Department of Energy's Office of Illness and Injury Prevention Programs, the IISP, and annual reports for DOE sites participating in this program can be found at:

http://www.hss.energy.gov/healthsafety/WSHP/epi/surv/

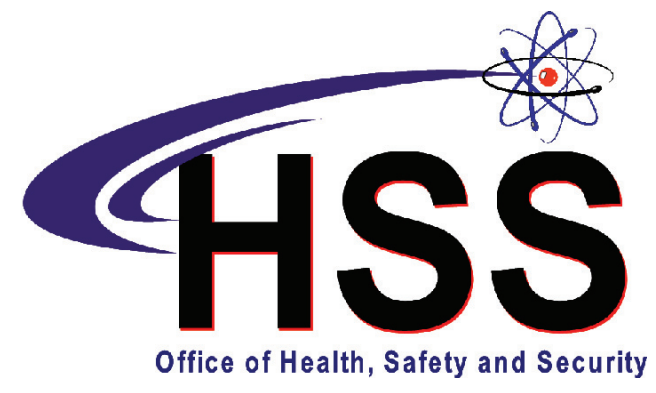

ACKNOWLEDGEMENT

LifeART images copyright 2000 Lippincott Williams \& Wilkins. All rights reserved.

This document was produced under contract number DE-AC05-06OR23100 between the U.S. Department of Energy and Oak Ridge Associated Universities. 


\title{
Nevada Test Site 2006 Illness and Injury Surveillance Report
}

\author{
At A Glance
}

A total of 3,960 NTS employees were included in illness and injury surveillance in 2006. There were 1,126 (28 percent) women and 2,834 (72 percent) men in the work force. The number of workers at NTS has steadily declined since 2003. The 2006 work force has 10 percent fewer workers than in 2003.

There were 182 absences among 146 women, resulting in an absence rate of 16.2 per 100 workers $(182 / 1,126)$. Among the 2,834 men, there were 276 absences, resulting in an absence rate of 9.7 per 100 workers $(276 / 2,834)$.

Security workers had the highest absence rate among men, 14.8 per 100 workers $(61 / 411)$. Among women, Crafts workers had the highest rate, 26.8 per 100 workers $(22 / 82)$.

The absence rate was 11.6 per 100 workers in 2006 , which is a 65 percent increase since 2003. This increase in the rate of absences has also been observed at other sites participating in IISP over the period 1995 to 2004.

Among both men and women, musculoskeletal disorders were among the most common diagnoses reported since 2003. In addition, respiratory disorders were common among women and injuries were common among men.

Women lost 4,644 calendar days due to illness and injury. Respiratory diseases (10 percent), unspecified symptoms (17 percent), and musculoskeletal disorders (18 percent) accounted for 45 percent of all reported diagnoses among women.

Men lost 6,372 calendar days due to illness and injury. Forty-seven percent of all reported diagnoses among men were due to injuries (16 percent), musculoskeletal disorders (16 percent), and unspecified symptoms (15 percent).

Fourteen sentinel health event occupational (SHEO) diagnoses were reported in 2006. Ten diagnoses were for carpal tunnel syndrome. Five (50 percent) of the carpal tunnel syndrome diagnoses were reported by Crafts workers who comprised 18 percent of the work force.

\section{OSHA-Recordable Events}

Men reported 58 OSHA-recordable events in 2006 and women reported 14 events. The rate of OSHA events was 2 per 100 workers among men and 1 per 100 workers among women. 
The rate of OSHA events has increased from 1 per 100 workers in 2003 to 2 per 100 workers in 2006, which is contrary to the decreasing rates seen among other sites participating in IISP during the period 1995 to 2004.

Occupational illnesses and injuries resulted in a total of 1,748 lost or restricted workdays reported by NTS in 2006. Crafts workers had the highest rate of OSHA events among women (9.8 per 100 workers), while Security and Fire workers had the highest rate among men (5.4 per 100 workers).

Injuries accounted for at least 50 percent of diagnoses for both men and women, with the most common type being unspecified injuries and sprains and strains, respectively.

The accidents most commonly reported among men and women were overexertion and strenuous movements and being struck by an object.

When the rate for OSHA-recordable injuries was considered separately, the highest rates were among Crafts workers for both men and women. This occupational group comprised 18 percent of the work force but reported 49 percent of the OSHA events. 
The Nevada Test Site Work Force - 2006

The Work Force by Gender and Age 1

The Work Force by Gender and Job

Category .... $\ldots 1$

Number and Length of Absences

Absence Rate by Gender and Age .2

Number of Days Absent by

Gender and Age ...2

Absence Rate by Job Category

and Gender. .3

Average Duration of Absence by

Job Category and Gender... ...3

\section{Diagnostic Categories}

Number of Diagnoses and Lost Calendar Days by Diagnostic Category

(Categorized by ICD-9-CM) and Gender......4

Common Diagnoses Among Female

Workers in 2006

Common Diagnoses Among Male

Workers in 2006

Number of Most Frequently Reported

Diagnoses by Job Category and Gender ......7

\section{Rates of Disease Occurrence}

Rates for All Illnesses and Injuries

Combined by Job Category, Gender,

and Age

Rates for Selected Diagnostic Categories by Job Category, Gender, and Age... .8

\section{Time Trends}

Age-Adjusted Rates for All Diagnoses

Combined Among Women and Men

from 2003 to 2006 10
Age-Adjusted Rates for Selected Diagnostic Categories Among Women and Men from

2003 to 2006 ..................................... 11

Age-Adjusted Rates for All Diagnoses

Combined Among Women and Men by

Job Category from 2003 to 2006 .... 12

\section{Sentinel Health Events for Occupations (SHEOs)}

Characteristics of SHEOs by Gender. 13

SHEO Diagnoses by Gender 13

Occupational Safety and Health Administration (OSHA)-Recordable Events

OSHA-Recordable Events by Gender and Age 14

OSHA-Recordable Events by Job

Category and Gender .... 14

Diagnostic and Accident Categories for OSHA-Recordable Events

OSHA-Recordable Diagnoses by

Diagnostic Category and Gender 15

OSHA-Recordable Accidents by Type

and Gender. 16

\section{Rates of OSHA-Recordable Events}

OSHA-Recordable Rates by Age and

Job Categories Among Women, All

Diagnoses Combined .17

OSHA-Recordable Rates by Age and Job Categories Among Men, All

Diagnoses Combined 17

Time Trends for OSHA-Recordable Events

Age-Adjusted Rates for All OSHA-Recordable Diagnoses Combined Among Women and Men by Job Category from 2003 to $2006 \ldots 18$

\section{Appendices}

Appendices A-W. .20 
The Nevada Test Site Work Force - 2006

Figure 1. The Work Force by Gender and Age

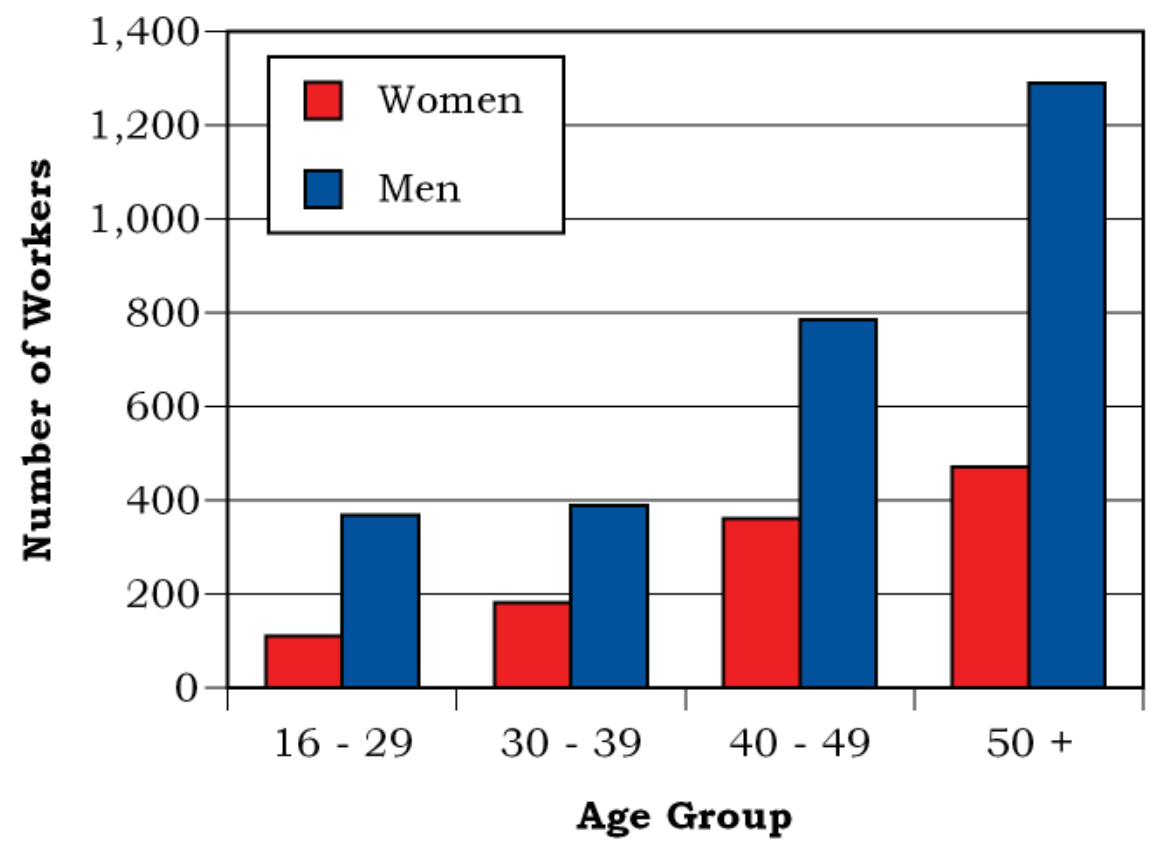

Figure 2. The Work Force by Gender and Job Category

\begin{tabular}{|l|c|c|}
\hline \multirow{2}{*}{ Job Category } & Women & Men \\
\hline \multirow{2}{*}{ Professional } & $\mathbf{5 5 0}$ & $\mathbf{1 , 2 6 9}$ \\
& $49 \%$ & $45 \%$ \\
\hline \multirow{2}{*}{ Administrative Support } & 302 & $\mathbf{7 3}$ \\
& $27 \%$ & $3 \%$ \\
\hline \multirow{2}{*}{ Technical Support } & 151 & 401 \\
& $13 \%$ & $14 \%$ \\
\hline \multirow{2}{*}{ Service } & 25 & 50 \\
& $2 \%$ & $2 \%$ \\
\hline \multirow{2}{*}{ Security and Fire } & 16 & 411 \\
\hline \multirow{2}{*}{ Crafts } & $2 \%$ & $14 \%$ \\
\hline \multirow{2}{*}{ Line Operators } & $\mathbf{8 2}$ & 629 \\
& $\mathbf{7} \%$ & $22 \%$ \\
\hline \multirow{2}{*}{ Total } & 0 & 1 \\
& $0 \%$ & $<1 \%$ \\
\hline
\end{tabular}


Number and Length of Absences

Figure 3. Absence Rate by Gender and Age

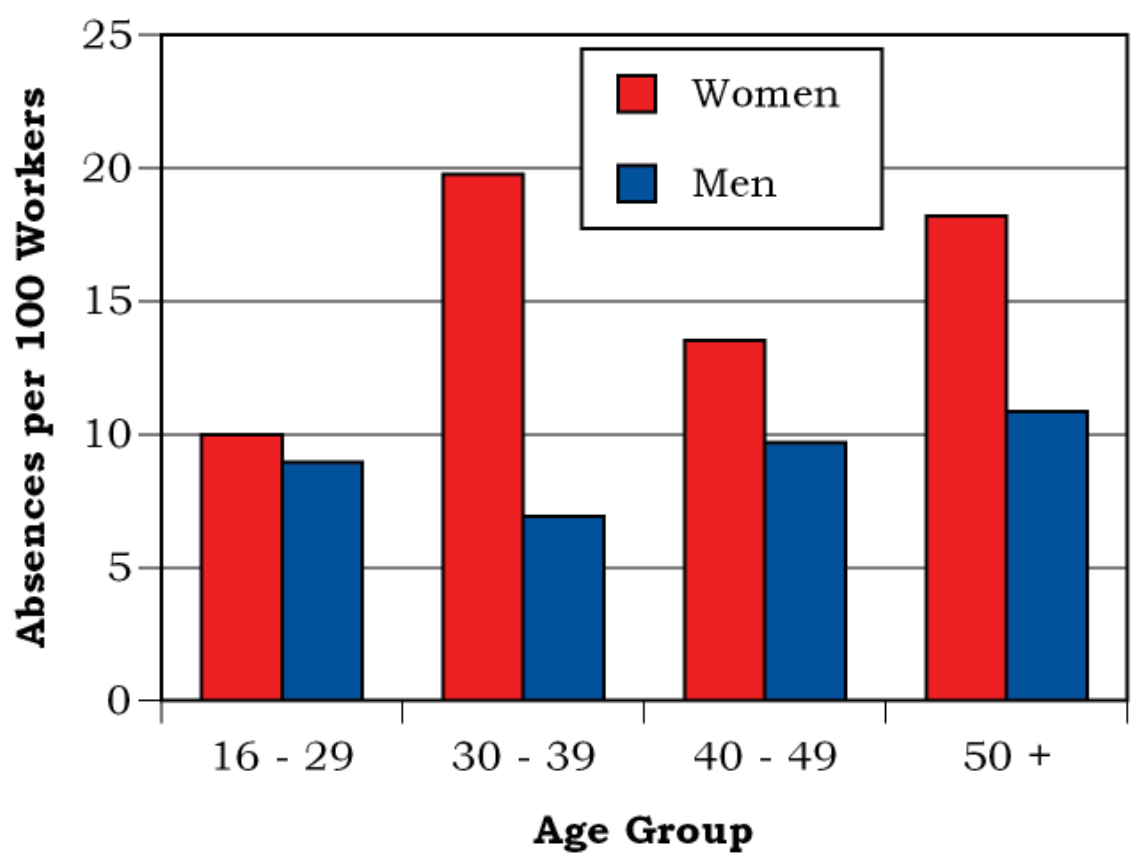

Figure 4. Number of Days Absent by Gender and Age

\begin{tabular}{|c|c|c|c|c|}
\hline \multirow{3}{*}{ Gender } & \multirow{2}{*}{ Age } & Number of & \multicolumn{2}{|c|}{ Number of Days Absent } \\
\cline { 3 - 5 } & & Absences & Total & Average \\
\hline \multirow{4}{*}{ Women } & $16-29$ & 11 & 103 & 9 \\
\cline { 2 - 5 } & $30-39$ & 36 & 910 & 25 \\
\cline { 2 - 5 } & $40-49$ & 49 & 1,041 & 21 \\
\cline { 2 - 5 } & $50+$ & 86 & 2,590 & 30 \\
\cline { 2 - 5 } & Total & 182 & 4,644 & 26 \\
\hline \multirow{4}{*}{ Men } & $16-29$ & 33 & 515 & 16 \\
\cline { 2 - 5 } & $30-39$ & 27 & 505 & 19 \\
\cline { 2 - 5 } & $40-49$ & 76 & 1,695 & 22 \\
\cline { 2 - 5 } & $50+$ & 140 & 3,657 & 26 \\
\cline { 2 - 5 } & Total & 276 & 6,372 & 23 \\
\hline
\end{tabular}


Figure 5. Absence Rate by Job Category and Gender

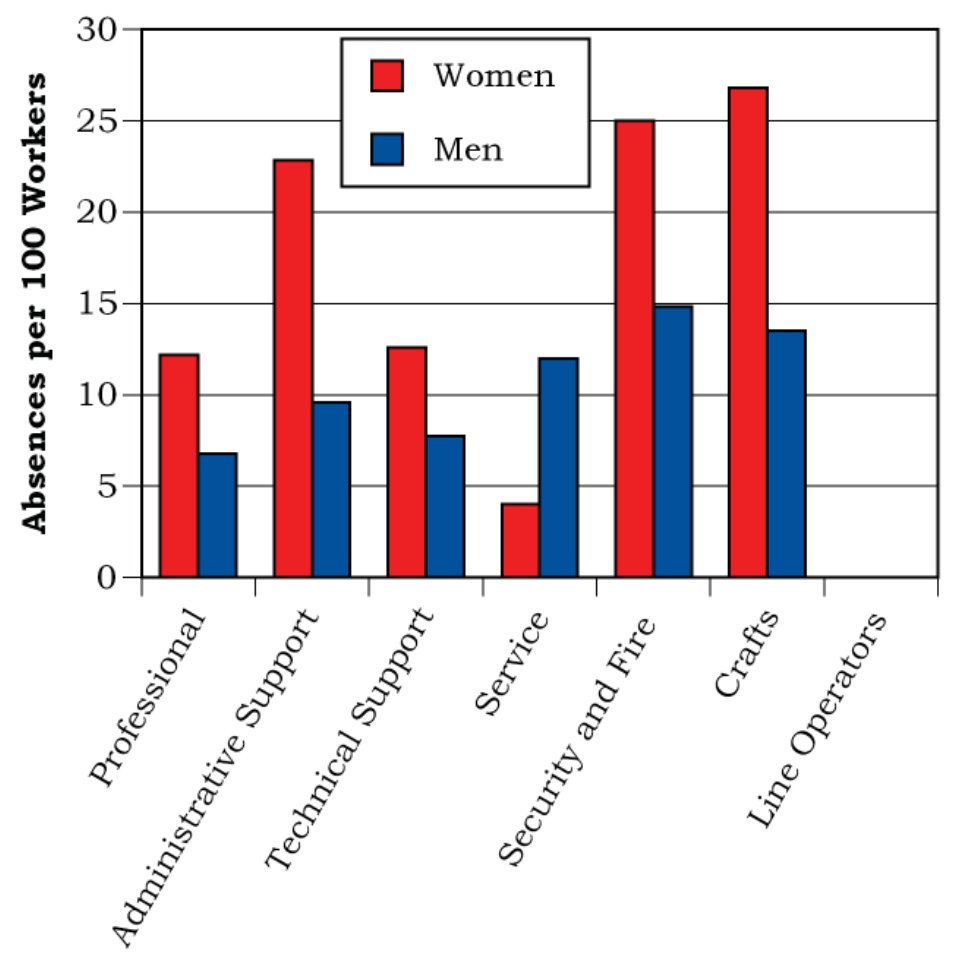

Job Category

Figure 6. Average Duration of Absence by Job Category and Gender

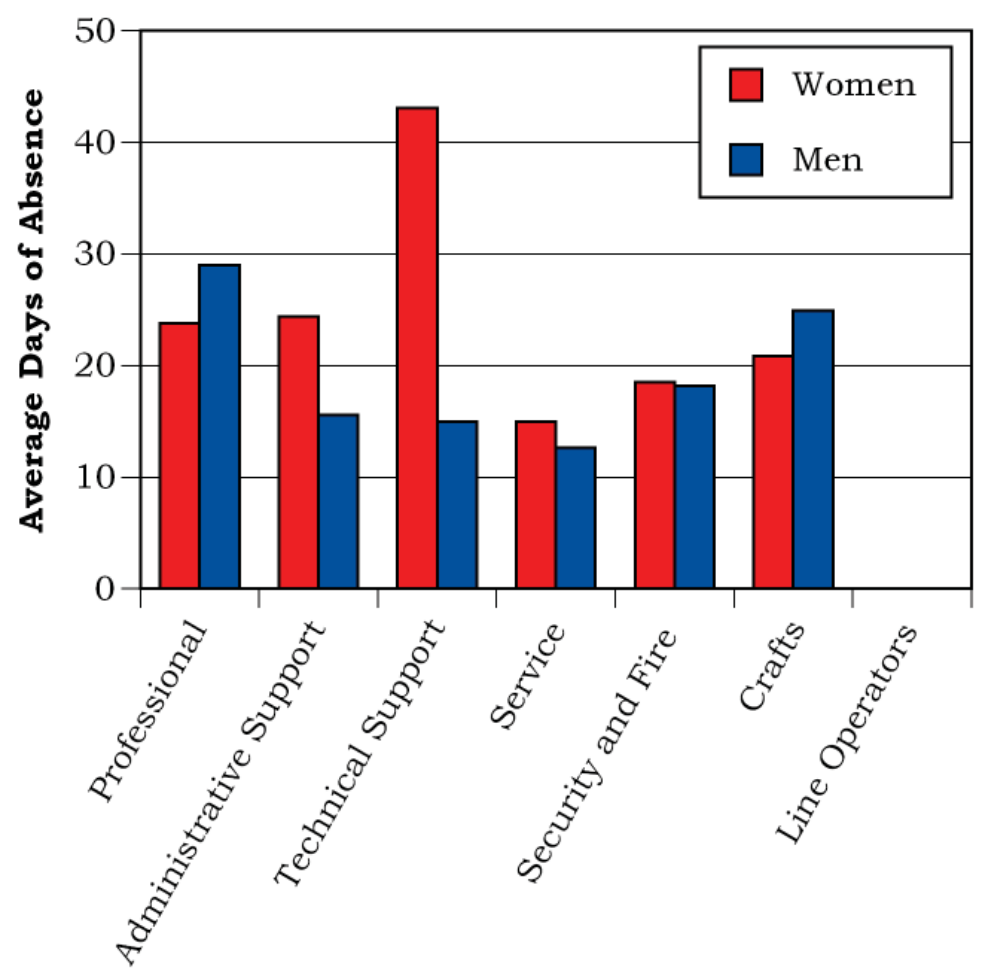

Job Category 


\section{Diagnostic Categories}

\section{Figure 7. Number of Diagnoses and Lost Calendar Days by Diagnostic Category (Categorized by ICD-9-CM) and Gender}

\begin{tabular}{|c|c|c|c|c|}
\hline \multirow[b]{2}{*}{ Diagnostic Category } & \multicolumn{2}{|c|}{ Women } & \multicolumn{2}{|c|}{ Men } \\
\hline & $\begin{array}{l}\text { Number of } \\
\text { Diagnoses }\end{array}$ & $\begin{array}{c}\text { Number } \\
\text { of Lost } \\
\text { Calendar } \\
\text { Days }\end{array}$ & $\begin{array}{l}\text { Number of } \\
\text { Diagnoses }\end{array}$ & $\begin{array}{c}\text { Number } \\
\text { of Lost } \\
\text { Calendar } \\
\text { Days }\end{array}$ \\
\hline Benign Growths & 10 & 366 & 3 & 118 \\
\hline Blood & 2 & 19 & 2 & 35 \\
\hline Cancer & 4 & 521 & 9 & 305 \\
\hline Digestive & 24 & 377 & 51 & 736 \\
\hline Endocrine/Metabolic & 13 & 162 & 19 & 503 \\
\hline Existing Birth Condition & 0 & 0 & 1 & 12 \\
\hline Genitourinary & 31 & 796 & 14 & 156 \\
\hline Heart/Circulatory & 17 & 347 & 47 & 839 \\
\hline Infections/Parasites & 10 & 145 & 15 & 125 \\
\hline Injury & 31 & 627 & 87 & 2,062 \\
\hline Miscarriage & 0 & 0 & NA & NA \\
\hline Musculoskeletal & 62 & 1,084 & 85 & 2,235 \\
\hline Nervous System & 18 & 437 & 37 & 344 \\
\hline Psychological & 18 & 668 & 16 & 410 \\
\hline Respiratory & 33 & 256 & 64 & 469 \\
\hline Skin & 5 & 135 & 10 & 103 \\
\hline Unspecified Symptoms & 57 & 730 & 81 & 785 \\
\hline
\end{tabular}

Note: Lost calendar days for each absence are counted more than once when multiple diagnoses occur in different diagnostic categories for the same absence. 
Figure 8. Common Diagnoses Among Female Workers in 2006

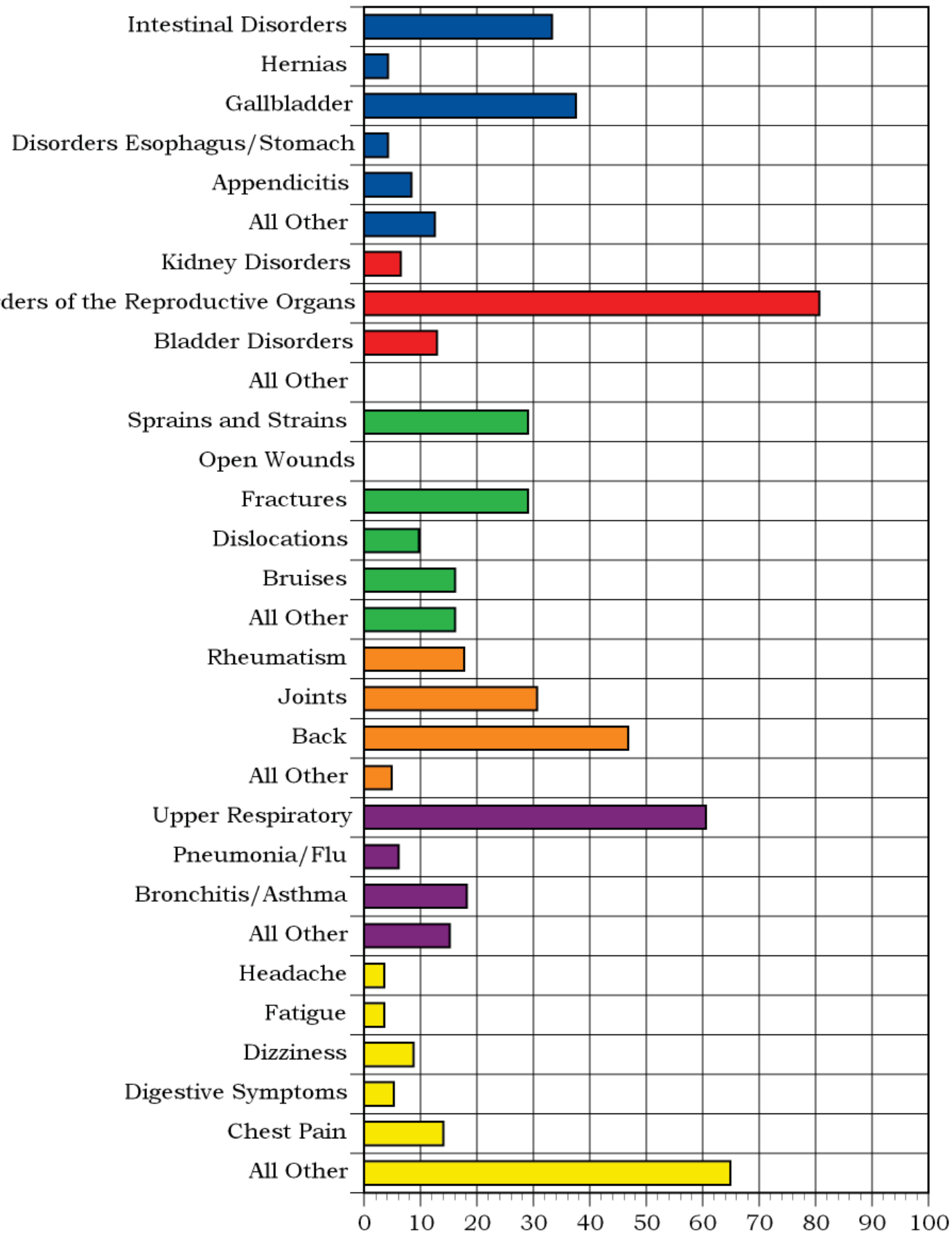

Percent Distribution of Diagnoses Within Diagnostic Category

Digestive, 24 Diagnoses

Genitourinary, 31 Diagnoses

Injury, 31 Diagnoses
Musculoskeletal, 62 Diagnoses

Respiratory, 33 Diagnoses

Unspecified Symptoms, 57 Diagnoses 
Figure 9. Common Diagnoses Among Male Workers in 2006

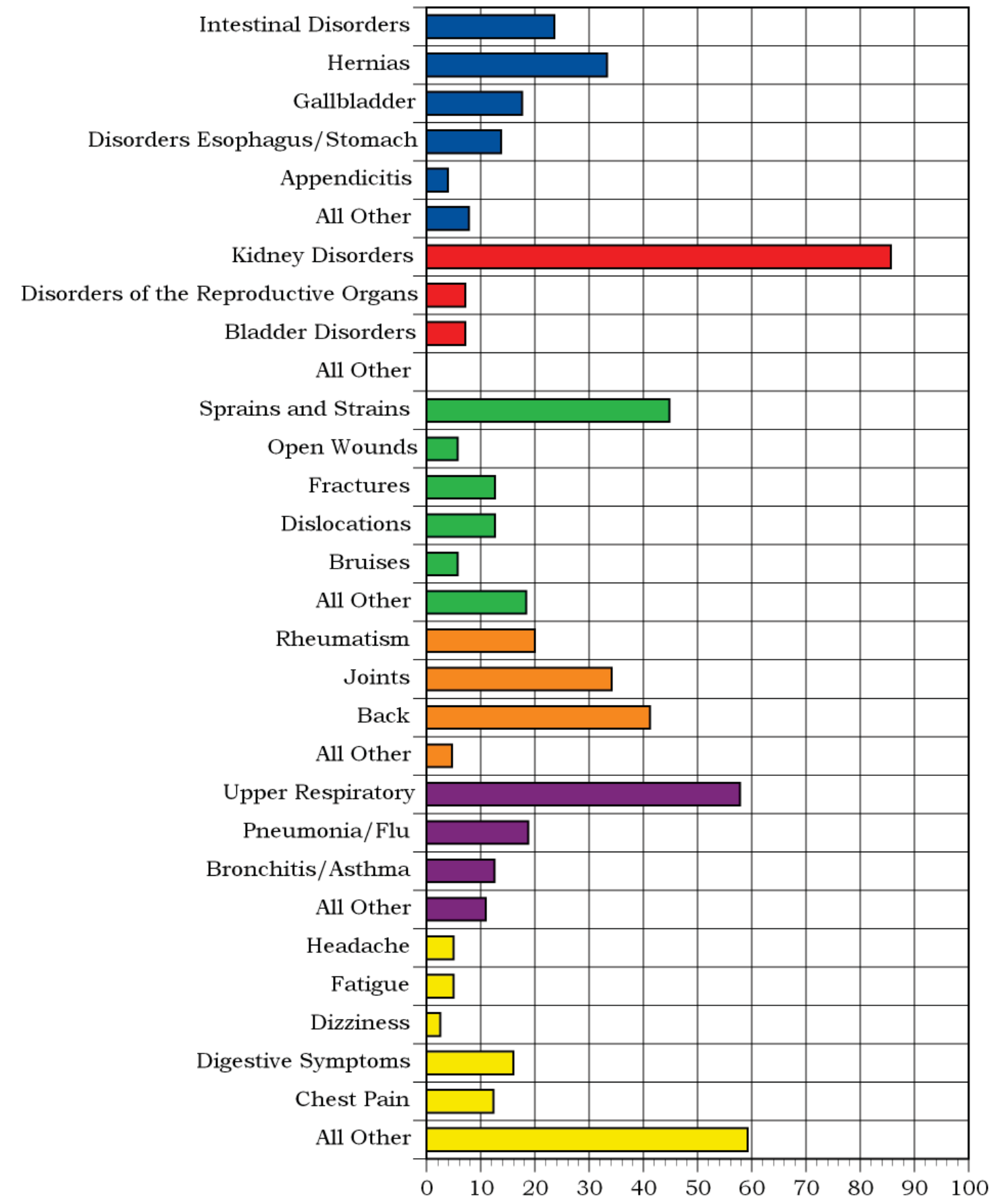

Percent Distribution of Diagnoses Within Diagnostic Category

Digestive, 51 Diagnoses

Genitourinary, 14 Diagnoses

Injury, 87 Diagnoses
Musculoskeletal, 85 Diagnoses

Respiratory, 64 Diagnoses

Unspecified Symptoms, 81 Diagnoses 


\section{Figure 10. Number of Most Frequently Reported Diagnoses by Job Category and Gender}

\begin{tabular}{|c|c|c|c|c|}
\hline Job Category & Men & & Women & \\
\hline \multirow{3}{*}{ Professional } & Musculoskeletal & 25 & Musculoskeletal & 31 \\
\hline & Heart/Circulatory & 22 & Unspecified Symptoms & 20 \\
\hline & Digestive & 19 & Genitourinary & 13 \\
\hline \multirow{3}{*}{ Administrative Support } & Unspecified Symptoms & 5 & Unspecified Symptoms & 21 \\
\hline & Respiratory & 4 & Genitourinary & 16 \\
\hline & Nervous System & 3 & Musculoskeletal & 15 \\
\hline \multirow{4}{*}{ Technical Support } & Unspecified Symptoms & 15 & Unspecified Symptoms & 8 \\
\hline & Digestive & 12 & Digestive & 4 \\
\hline & Heart/Circulatory & 7 & Musculoskeletal & 4 \\
\hline & Musculoskeletal & 7 & & \\
\hline \multirow{3}{*}{ Service } & Heart/Circulatory & 3 & Respiratory & 1 \\
\hline & Injury & 3 & & \\
\hline & Unspecified Symptoms & 3 & & \\
\hline \multirow{4}{*}{ Security and Fire } & Injury & 27 & Respiratory & 3 \\
\hline & Musculoskeletal & 24 & Heart/Circulatory & 1 \\
\hline & Respiratory & 21 & Nervous System & 1 \\
\hline & Unspecified Symptoms & 21 & Unspecified Symptoms & 1 \\
\hline \multirow{3}{*}{ Crafts } & Injury & 33 & Musculoskeletal & 12 \\
\hline & Musculoskeletal & 26 & Injury & 11 \\
\hline & Unspecified Symptoms & 24 & Unspecified Symptoms & 7 \\
\hline Line Operators & & 0 & & 0 \\
\hline
\end{tabular}




\section{Rates of Disease Occurrence}

Figure 11. Rates for All Illnesses and Injuries Combined by Job Category, Gender, and Age

\begin{tabular}{|c|c|c|c|c|}
\hline \multirow{2}{*}{$\begin{array}{c}\text { All Illnesses \& } \\
\text { Injuries Combined }\end{array}$} & \multicolumn{4}{|c|}{ Rate per 1,000} \\
\hline & Job Category & Age & Men & Women \\
\hline & \multirow{2}{*}{ Professional } & $<50$ & 75 & 279 \\
\hline & & $50+$ & 164 & 178 \\
\hline & \multirow{2}{*}{ Administrative Support } & $<50$ & 277 & 304 \\
\hline & & $50+$ & 115 & 511 \\
\hline & \multirow{2}{*}{ Technical Support } & $<50$ & 126 & 130 \\
\hline & & $50+$ & 173 & 230 \\
\hline & \multirow{2}{*}{ Service } & $<50$ & 696 & $\mathbf{0}$ \\
\hline & & $50+$ & 37 & 111 \\
\hline & \multirow{2}{*}{ Security and Fire } & $<50$ & 265 & 286 \\
\hline & & $50+$ & 604 & 1,000 \\
\hline & \multirow{2}{*}{ Crafts } & $<50$ & 288 & 286 \\
\hline & & $50+$ & 235 & 1,030 \\
\hline & \multirow{2}{*}{ Line Operators } & $<50$ & $\mathbf{0}$ & 0 \\
\hline & & $50+$ & $\mathbf{0}$ & 0 \\
\hline
\end{tabular}

Figure 12. Rates for Selected Diagnostic Categories by Job Category, Gender, and Age

\begin{tabular}{|c|c|c|c|c|}
\hline \multirow{2}{*}{ Cancer } & \multicolumn{4}{|c|}{ Rate per 1,000} \\
\hline & Job Category & Age & Men & Women \\
\hline & \multirow{2}{*}{ Professional } & $<50$ & 0 & 3 \\
\hline & & $50+$ & 9 & 5 \\
\hline & \multirow{2}{*}{ Administrative Support } & $<50$ & 0 & 0 \\
\hline & & $50+$ & 0 & 0 \\
\hline & \multirow{2}{*}{ Technical Support } & $<50$ & 0 & 0 \\
\hline & & $50+$ & 0 & 27 \\
\hline & \multirow{2}{*}{ Service } & $<50$ & 0 & 0 \\
\hline & & $50+$ & 0 & 0 \\
\hline & \multirow{2}{*}{ Security and Fire } & $<50$ & 0 & 0 \\
\hline & & $50+$ & 38 & 0 \\
\hline & \multirow{2}{*}{ Crafts } & $<50$ & 0 & 0 \\
\hline & & $50+$ & 3 & 0 \\
\hline & \multirow{2}{*}{ Line Operators } & $<50$ & 0 & 0 \\
\hline & & $50+$ & 0 & 0 \\
\hline
\end{tabular}




\section{Figure 12. Rates for Selected Diagnostic Categories by Job Category, Gender, and Age (Continued)}

\begin{tabular}{|c|c|c|c|c|}
\hline \multirow{2}{*}{ Heart/Circulatory } & \multicolumn{4}{|c|}{ Rate per 1,000} \\
\hline & Job Category & Age & Men & Women \\
\hline & \multirow{2}{*}{ Professional } & $<50$ & 14 & 12 \\
\hline & & $50+$ & 20 & 0 \\
\hline & \multirow{2}{*}{ Administrative Support } & $<50$ & 21 & 19 \\
\hline & & $50+$ & 0 & 35 \\
\hline & \multirow{2}{*}{ Technical Support } & $<50$ & 9 & 0 \\
\hline & & $50+$ & 28 & 14 \\
\hline & \multirow{2}{*}{ Service } & $<50$ & 130 & 0 \\
\hline & & $50+$ & 0 & 0 \\
\hline & \multirow{2}{*}{ Security and Fire } & $<50$ & 8 & 0 \\
\hline & & $50+$ & 0 & 500 \\
\hline & \multirow{2}{*}{ Crafts } & $<50$ & 3 & 0 \\
\hline & & $50+$ & 32 & 91 \\
\hline & \multirow{2}{*}{ Line Operators } & $<50$ & 0 & 0 \\
\hline & & $50+$ & 0 & 0 \\
\hline
\end{tabular}

\begin{tabular}{|c|c|c|c|c|}
\hline \multirow{2}{*}{ Respiratory } & \multicolumn{4}{|c|}{ Rate per 1,000} \\
\hline & Job Category & Age & Men & Women \\
\hline & \multirow{2}{*}{ Professional } & $<50$ & 2 & 21 \\
\hline & & $50+$ & 19 & 14 \\
\hline & \multirow{2}{*}{ Administrative Support } & $<50$ & 85 & 31 \\
\hline & & $50+$ & 0 & 57 \\
\hline & \multirow{2}{*}{ Technical Support } & $<50$ & 18 & 26 \\
\hline & & $50+$ & 0 & 0 \\
\hline & \multirow{2}{*}{ Service } & $<50$ & 0 & $\mathbf{0}$ \\
\hline & & $50+$ & 0 & 111 \\
\hline & \multirow{2}{*}{ Security and Fire } & $<50$ & 42 & 214 \\
\hline & & $50+$ & 113 & 0 \\
\hline & \multirow{2}{*}{ Crafts } & $<50$ & 44 & 20 \\
\hline & & $50+$ & 23 & 91 \\
\hline & \multirow{2}{*}{ Line Operators } & $<50$ & 0 & 0 \\
\hline & & $50+$ & 0 & $\mathbf{0}$ \\
\hline
\end{tabular}


Figure 12. Rates for Selected Diagnostic Categories by Job Category, Gender, and Age (Continued)

\begin{tabular}{|c|c|c|c|c|}
\hline \multirow{2}{*}{ Injury } & \multicolumn{4}{|c|}{ Rate per 1,000} \\
\hline & Job Category & Age & Men & Women \\
\hline & \multirow{2}{*}{ Professional } & $<50$ & 7 & 24 \\
\hline & & $50+$ & 20 & 5 \\
\hline & \multirow{2}{*}{ Administrative Support } & $<50$ & 0 & 19 \\
\hline & & $50+$ & 0 & 43 \\
\hline & \multirow{2}{*}{ Technical Support } & $<50$ & 5 & 0 \\
\hline & & $50+$ & 28 & 27 \\
\hline & \multirow{2}{*}{ Service } & $<50$ & 130 & 0 \\
\hline & & $50+$ & 0 & 0 \\
\hline & \multirow{2}{*}{ Security and Fire } & $<50$ & 61 & 0 \\
\hline & & $50+$ & 94 & 0 \\
\hline & \multirow{2}{*}{ Crafts } & $<50$ & 60 & 102 \\
\hline & & $50+$ & 45 & 182 \\
\hline & \multirow{2}{*}{ Line Operators } & $<50$ & 0 & 0 \\
\hline & & $50+$ & 0 & 0 \\
\hline
\end{tabular}

\section{Time Trends}

Figure 13. Age-Adjusted Rates for All Diagnoses Combined Among Women and Men from 2003 to 2006*

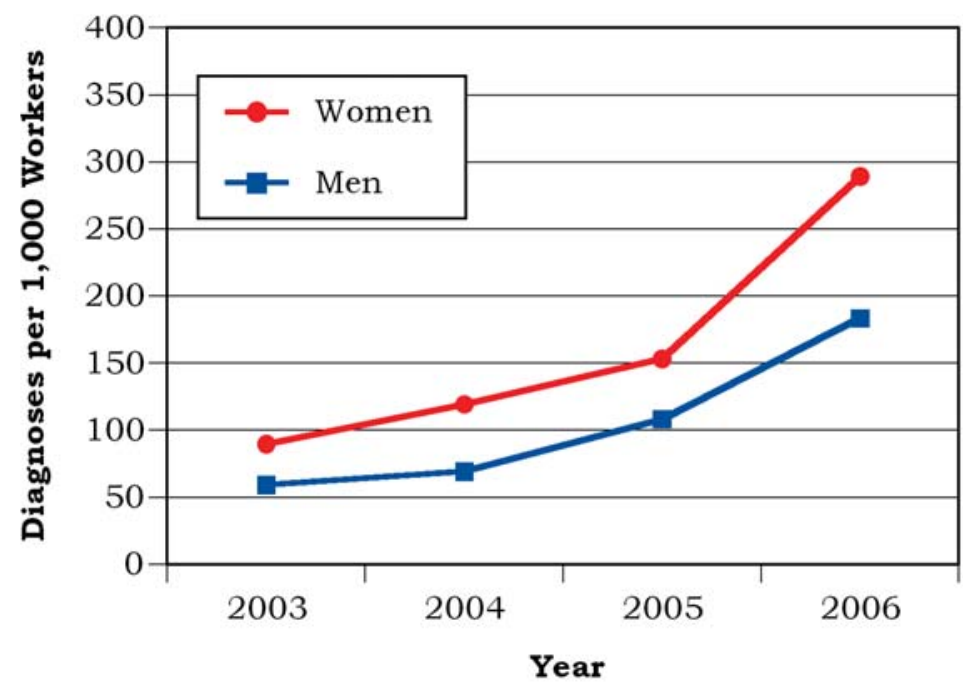

* Standardized to age distribution of 2000 U.S. population. 
Figure 14. Age-Adjusted Rates for Selected Diagnostic Categories Among Women and Men from 2003 to 2006*

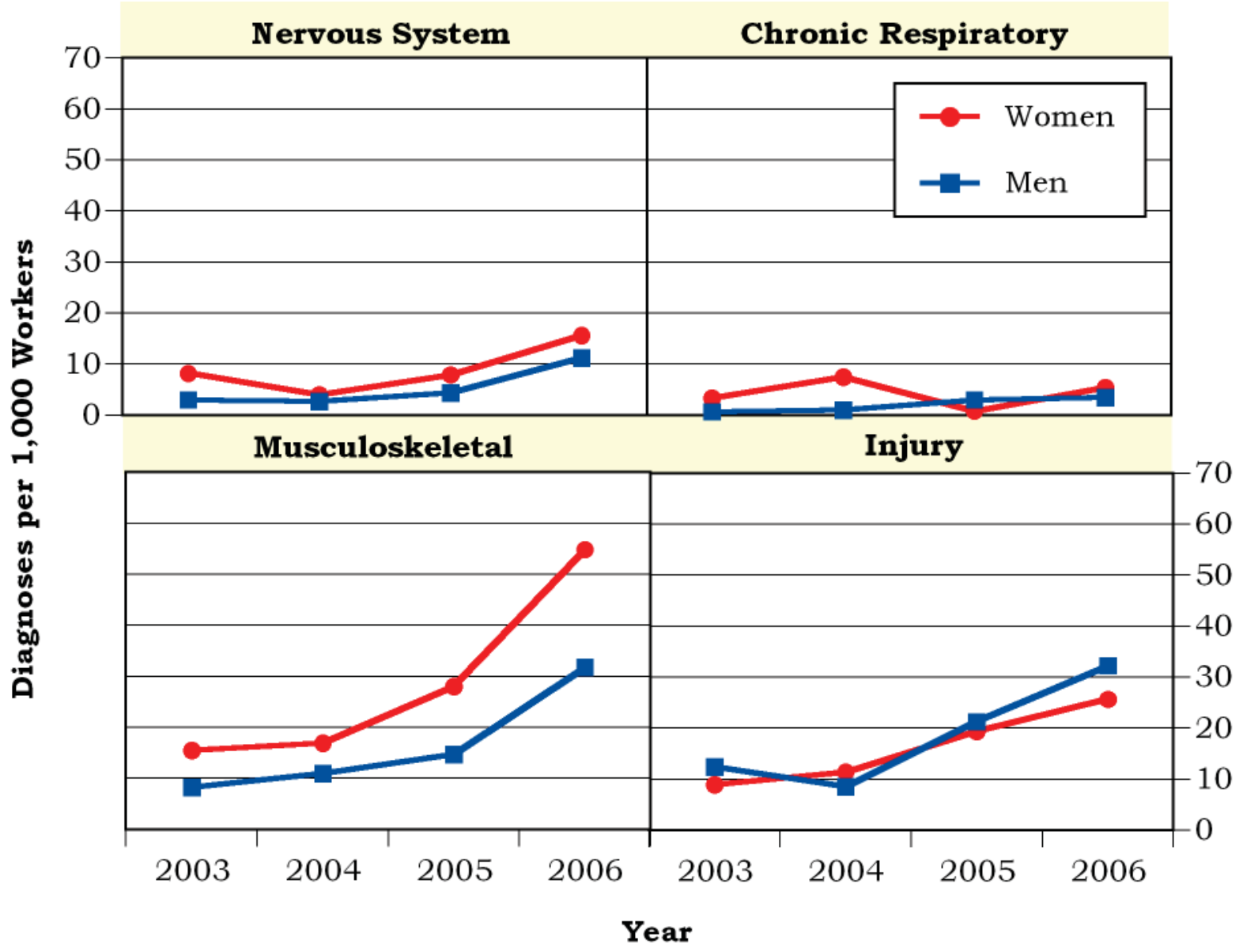

* Standardized to age distribution of 2000 U.S. population. 
Figure 15. Age-Adjusted Rates for All Diagnoses Combined Among Women and Men by Job Category from 2003 to 2006*

(Rates are shown only for job and gender categories in which there was at least 1 worker.)

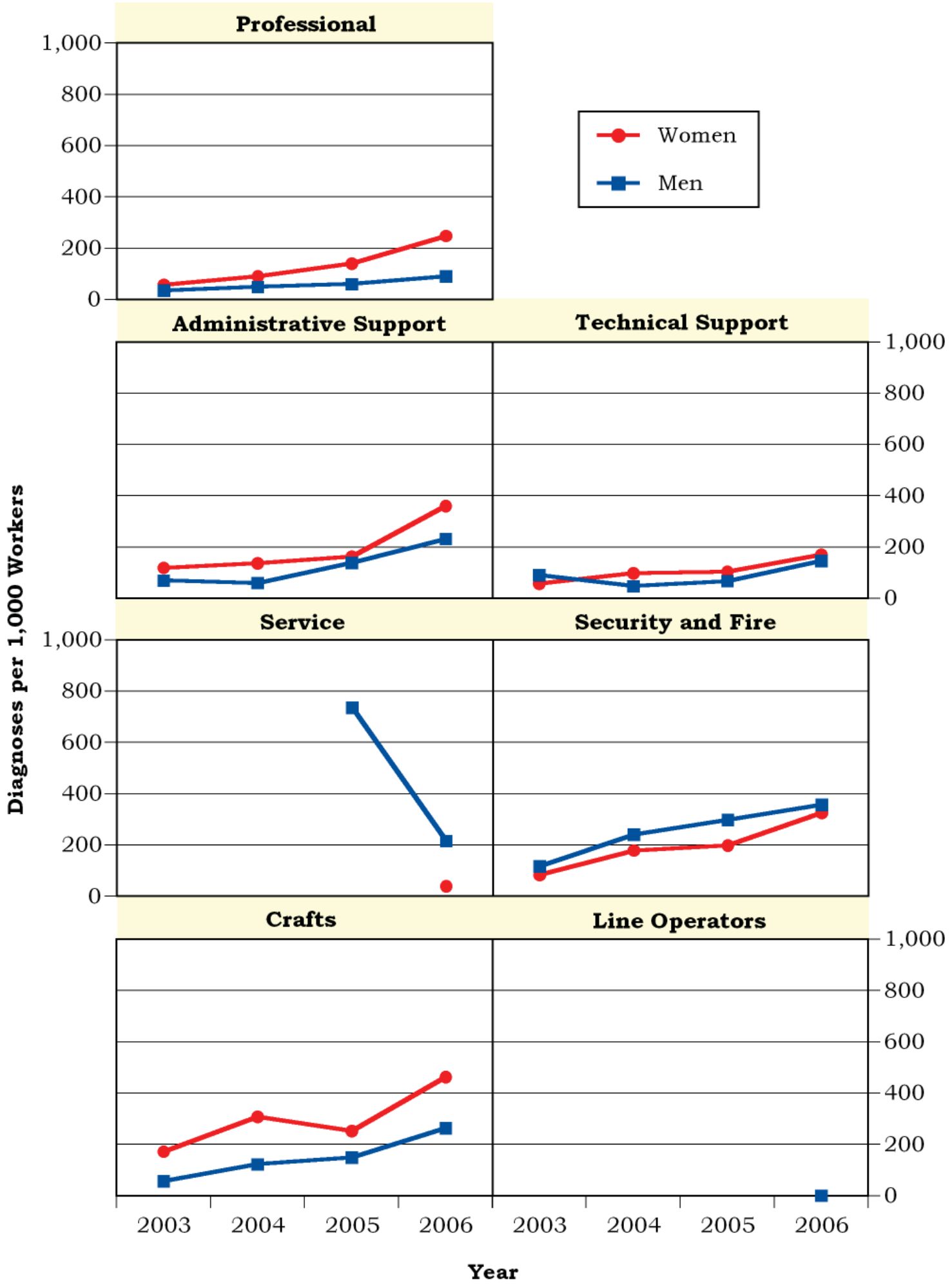

* Standardized to age distribution of 2000 U.S. population. 


\section{Sentinel Health Events for Occupations (SHEOs)}

An occupational sentinel health event (SHEO) is a disease, disability, or death that is likely to be occupationally related. Although sentinel health events may indicate an occupational exposure, many may result from nonoccupational exposures. Sentinel health events are therefore assessed in two categories:

Definite Sentinel Health Events: Diseases that are unlikely to occur in the absence of an occupational exposure (e.g., asbestosis).

Possible Sentinel Health Events: Diseases that may be occupational but can also occur in the absence of an occupational exposure (e.g., lung cancer or carpal tunnel syndrome).

Figure 16. Characteristics of SHEOs by Gender

\begin{tabular}{|l|c|c|c|c|}
\hline \multirow{2}{*}{} & \multicolumn{2}{|c|}{$\begin{array}{c}\text { Total Number of } \\
\text { SHEO Diagnoses }\end{array}$} & \multicolumn{2}{c|}{$\begin{array}{c}\text { Total Number of } \\
\text { Days Absent }\end{array}$} \\
\cline { 2 - 5 } & Men & Women & Men & Women \\
\hline Definite & 1 & 0 & 14 & 0 \\
\hline Possible & 8 & 5 & 113 & 217 \\
\hline Total & 9 & 5 & 127 & 217 \\
\hline
\end{tabular}

Figure 17. SHEO Diagnoses by Gender

\begin{tabular}{|l|c|c|}
\hline \multirow{2}{*}{\multicolumn{1}{|c|}{ Diagnoses }} & \multicolumn{2}{c|}{ Gender } \\
\cline { 2 - 3 } & Women & Men \\
\hline Carpal Tunnel Syndrome & 4 & 6 \\
\hline Pneumonconiosis (Berylliosis) & 0 & 0 \\
\hline Musculoskeletal Conditions & 0 & 0 \\
\hline Injuries & 0 & 0 \\
\hline Other Conditions & 1 & 3 \\
\hline
\end{tabular}


Occupational Safety and Health Administration (OSHA)-Recordable Events

Figure 18. OSHA-Recordable Events by Gender and Age

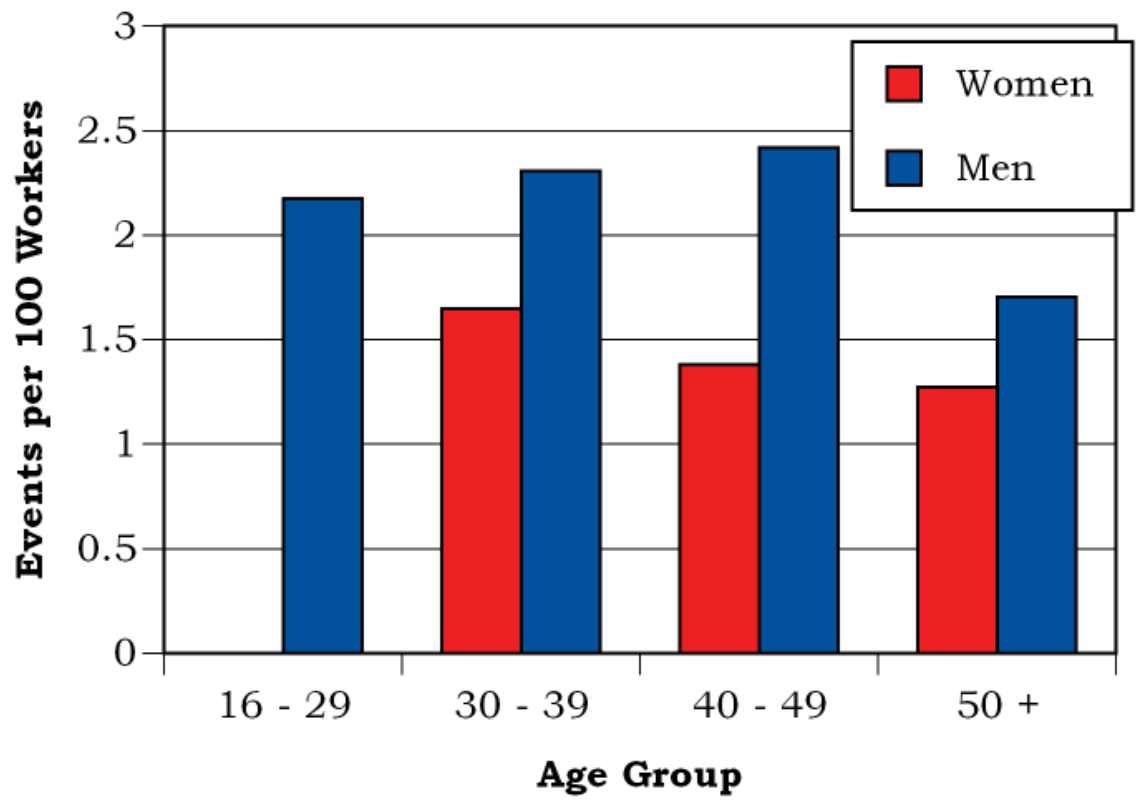

Figure 19. OSHA-Recordable Events by Job Category and Gender

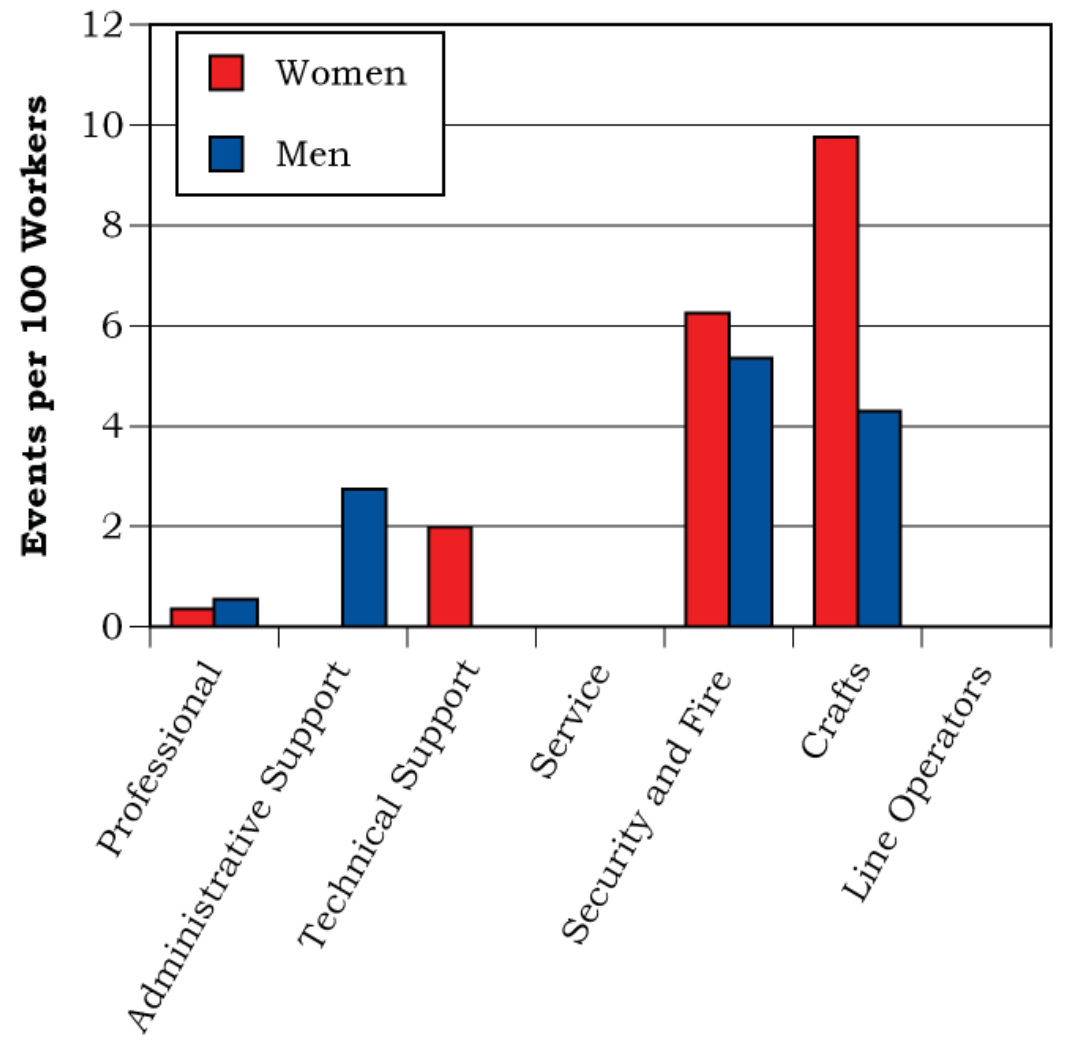

Job Category 


\section{Diagnostic and Accident Categories for OSHA-Recordable Events}

Figure 20. OSHA-Recordable Diagnoses by Diagnostic Category and Gender

\begin{tabular}{|l|c|c|}
\hline \multirow{2}{*}{ Diagnostic Category } & \multicolumn{2}{|c|}{ Gender } \\
\cline { 2 - 3 } & Women & Men \\
\hline Digestive & $\mathbf{0}$ & $\mathbf{1}$ \\
\hline Endocrine/Metabolic & $\mathbf{0}$ & $\mathbf{1}$ \\
\hline Genitourinary & $\mathbf{0}$ & $\mathbf{1}$ \\
\hline Musculoskeletal & $\mathbf{7}$ & $\mathbf{2 9}$ \\
\hline Nervous System & $\mathbf{0}$ & $\mathbf{5}$ \\
\hline Respiratory & $\mathbf{0}$ & $\mathbf{3}$ \\
\hline Unspecified Symptoms & $\mathbf{3}$ & $\mathbf{9}$ \\
\hline Injury & $\mathbf{1 5}$ & $\mathbf{4 9}$ \\
\hline Fractures - Upper Limb & $\mathbf{1}$ & $\mathbf{1}$ \\
\hline Fractures - Lower Limb & $\mathbf{0}$ & $\mathbf{1}$ \\
\hline Dislocations & $\mathbf{0}$ & $\mathbf{5}$ \\
\hline Back Sprains \& Strains & $\mathbf{1}$ & $\mathbf{3}$ \\
\hline Other Sprains \& Strains & $\mathbf{3}$ & $\mathbf{7}$ \\
\hline Open Wounds - Head, Neck, Trunk & $\mathbf{2}$ & $\mathbf{2}$ \\
\hline Open Wounds - Upper Limb & $\mathbf{0}$ & $\mathbf{3}$ \\
\hline Open Wounds - Lower Limb & $\mathbf{0}$ & $\mathbf{2}$ \\
\hline Superficial Injuries & $\mathbf{0}$ & $\mathbf{3}$ \\
\hline Bruises & $\mathbf{4}$ & $\mathbf{1}$ \\
\hline Foreign Bodies Entering Orifice & $\mathbf{1}$ & $\mathbf{2}$ \\
\hline Burns & $\mathbf{1}$ & $\mathbf{0}$ \\
\hline Unspecified Injuries & $\mathbf{2}$ & $\mathbf{1 8}$ \\
\hline Adverse Reactions to External Causes & $\mathbf{0}$ & $\mathbf{1}$ \\
\hline
\end{tabular}


Figure 21. OSHA-Recordable Accidents by Type and Gender

\begin{tabular}{|l|c|c|}
\hline \multirow{2}{*}{\multicolumn{2}{|c|}{ Accident Category }} & \multicolumn{2}{|c|}{ Gender } \\
\cline { 2 - 3 } & Women & Men \\
\cline { 2 - 3 } & $\begin{array}{c}\text { Number of } \\
\text { Accidents }\end{array}$ & $\begin{array}{c}\text { Number of } \\
\text { Accidents }\end{array}$ \\
\hline Motor Vehicle Traffic & $\mathbf{0}$ & $\mathbf{2}$ \\
\hline Falls & $\mathbf{3}$ & $\mathbf{8}$ \\
\hline Natural/Environmental Factors & $\mathbf{0}$ & $\mathbf{4}$ \\
\hline Submersion/Suffocation/Foreign Bodies & $\mathbf{1}$ & $\mathbf{2}$ \\
\hline Other Accidents & $\mathbf{1 0}$ & $\mathbf{3 8}$ \\
\hline Struck by an Object & $\mathbf{3}$ & $\mathbf{1 0}$ \\
\hline Caught Between Objects & $\mathbf{1}$ & $\mathbf{3}$ \\
\hline Cutting/Piercing Instrument/Object & $\mathbf{0}$ & $\mathbf{2}$ \\
\hline Firearm & $\mathbf{0}$ & $\mathbf{1}$ \\
\hline Hot, Corrosive, or Caustic Material/ & $\mathbf{1}$ & $\mathbf{0}$ \\
\hline Steam & & $\mathbf{2 2}$ \\
\hline Overexertion/Strenuous Movements & $\mathbf{3}$ & $\mathbf{0}$ \\
\hline Repetitive Trauma & $\mathbf{2}$ & $\mathbf{5 4}$ \\
\hline Total & $\mathbf{1 4}$ & \\
\hline
\end{tabular}




\section{Rates of OSHA-Recordable Events}

Figure 22. OSHA-Recordable Rates by Age and Job Categories Among Women, All Diagnoses Combined

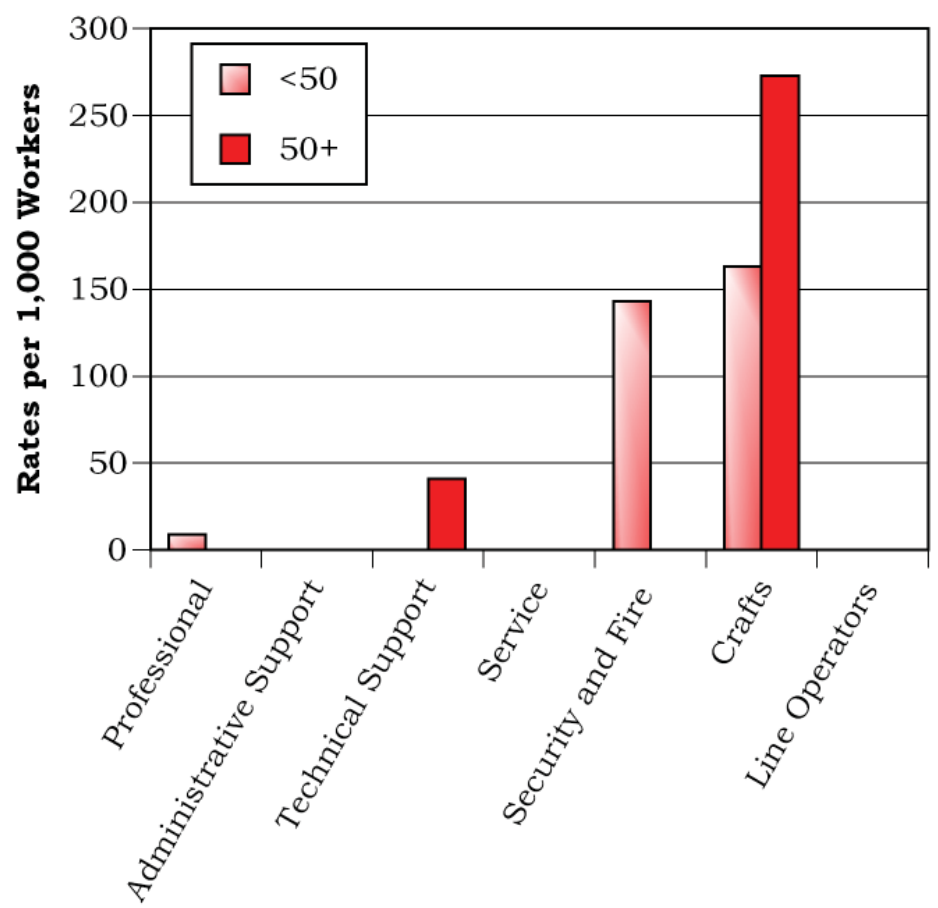

Job Category

Figure 23. OSHA-Recordable Rates by Age and Job Categories Among Men, All Diagnoses Combined

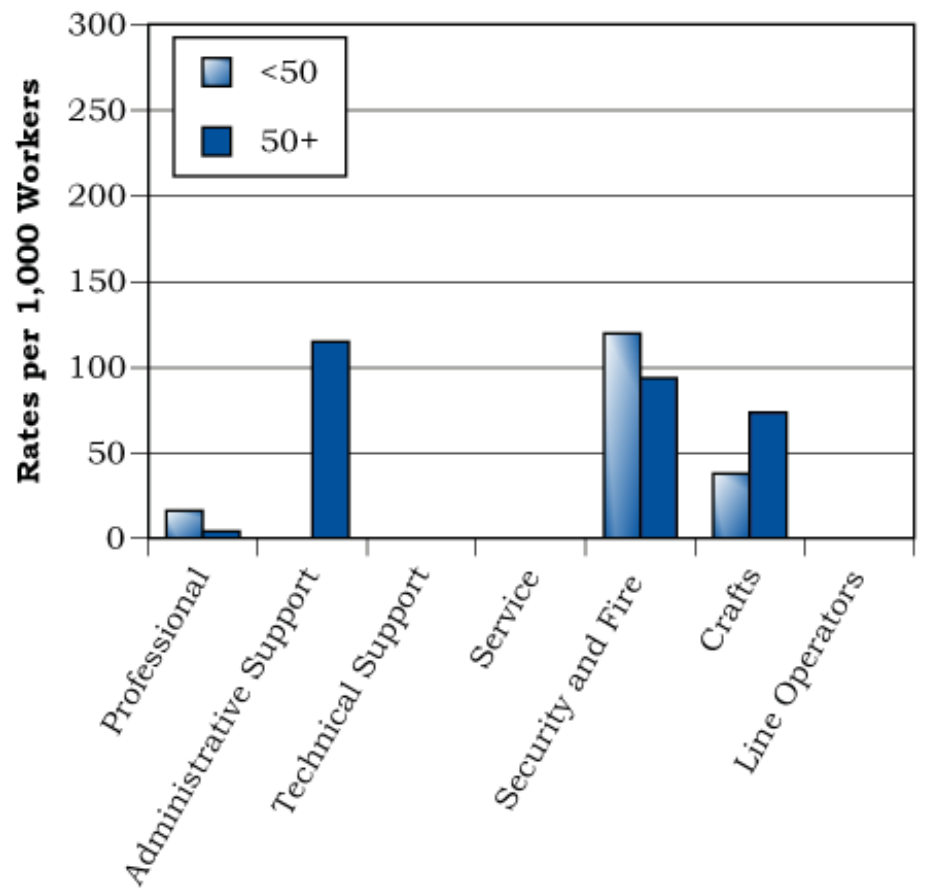

Job Category 


\section{Time Trends for OSHA-Recordable Events}

Figure 24. Age-Adjusted Rates for All OSHA-Recordable Diagnoses Combined Among Women and Men by Job Category from 2003 to 2006*

(Rates are shown only for job and gender categories in which there was at least 1 worker.)

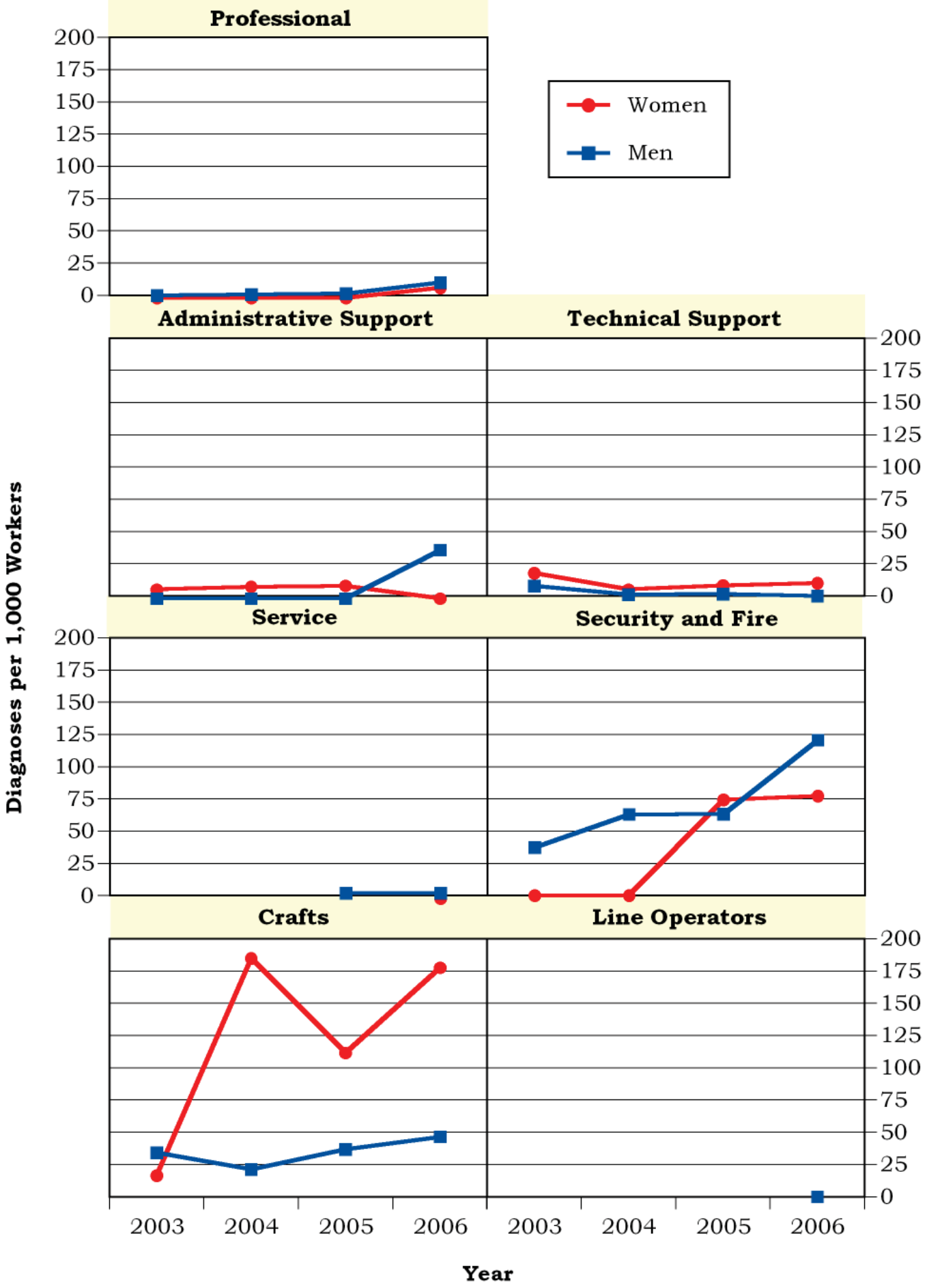

* Standardized to age distribution of 2000 U.S. population. 


\section{Appendices}


Nevada Test Site 2006

Absence Data

Appendix A. Work Force by Gender, Age, and Job Category

\begin{tabular}{|c|c|c|c|c|c|c|c|c|c|c|c|}
\hline \multirow{3}{*}{ Job Category } & \multicolumn{5}{|c|}{ Women } & \multicolumn{5}{|c|}{ Men } & \multirow[b]{3}{*}{ TOTAL } \\
\hline & \multicolumn{4}{|c|}{ Age Group } & \multirow[b]{2}{*}{ TOTAL } & \multicolumn{4}{|c|}{ Age Group } & \multirow[b]{2}{*}{ TOTAL } & \\
\hline & $16-29$ & 30 - 39 & 40 - 49 & $50+$ & & $16-29$ & $30-39$ & $40-49$ & $50+$ & & \\
\hline Professional & 52 & 99 & 186 & 213 & 550 & 90 & 155 & 328 & 696 & 1,269 & 1,819 \\
\hline Administrative Support & 31 & 50 & 80 & 141 & 302 & 17 & 11 & 19 & 26 & 73 & 375 \\
\hline Technical Support & 12 & 21 & 44 & 74 & 151 & 39 & 65 & 118 & 179 & 401 & 552 \\
\hline Service & 3 & 1 & 12 & 9 & 25 & 2 & 3 & 18 & 27 & 50 & 75 \\
\hline Security and Fire & 6 & 2 & 6 & 2 & 16 & 177 & 72 & 109 & 53 & 411 & 427 \\
\hline Crafts & 6 & 9 & 34 & 33 & 82 & 43 & 83 & 193 & 310 & 629 & 711 \\
\hline Line Operators & 0 & 0 & 0 & 0 & 0 & 0 & 1 & 0 & 0 & 1 & 1 \\
\hline TOTAL & 110 & 182 & 362 & 472 & 1,126 & 368 & 390 & 785 & 1,291 & 2,834 & 3,960 \\
\hline
\end{tabular}

Appendix B. Age Distribution of the Work Force by Gender

\begin{tabular}{|c|r|r|r|r|r|r|r|r|}
\hline \multirow{3}{*}{ Year } & \multicolumn{4}{|c|}{ Women } & \multicolumn{4}{c|}{ Men } \\
\cline { 2 - 9 } & \multicolumn{3}{|c|}{$\begin{array}{c}\text { Percent Distribution by Age } \\
\text { Group }\end{array}$} & \multicolumn{4}{c|}{$\begin{array}{c}\text { Percent Distribution by Age } \\
\text { Group }\end{array}$} \\
\cline { 2 - 9 } & $\mathbf{1 6}-\mathbf{2 9}$ & $\mathbf{3 0}-\mathbf{3 9}$ & $\mathbf{4 0}-\mathbf{4 9}$ & $\mathbf{5 0}+$ & $\mathbf{1 6}-\mathbf{2 9}$ & $\mathbf{3 0}-\mathbf{3 9}$ & $\mathbf{4 0}-\mathbf{4 9}$ & $\mathbf{5 0}+$ \\
\hline $\mathbf{2 0 0 2}$ & 12.28 & 21.63 & 31.98 & 34.11 & 8.38 & 15.54 & 29.99 & 46.09 \\
\hline $\mathbf{2 0 0 3}$ & 10.17 & 20.71 & 33.53 & 35.59 & 8.64 & 15.36 & 29.47 & 46.53 \\
\hline $\mathbf{2 0 0 4}$ & 11.39 & 19.32 & 31.41 & 37.88 & 10.98 & 14.32 & 28.90 & 45.80 \\
\hline $\mathbf{2 0 0 5}$ & 11.03 & 17.20 & 33.09 & 38.68 & 11.30 & 14.06 & 29.01 & 45.62 \\
\hline $\mathbf{2 0 0 6}$ & 9.77 & 16.16 & 32.15 & 41.92 & 12.99 & 13.76 & 27.70 & 45.55 \\
\hline
\end{tabular}


Nevada Test Site 2006

Absence Data

Appendix C. Total Number of Workers Who Reported at Least One Absence by Gender, Age, and Job Category*

\begin{tabular}{|c|c|c|c|c|c|c|c|c|c|c|c|}
\hline \multirow{3}{*}{ Job Category } & \multicolumn{5}{|c|}{ Women } & \multicolumn{5}{|c|}{ Men } & \multirow[b]{3}{*}{ TOTAL } \\
\hline & \multicolumn{4}{|c|}{ Age Group } & \multirow[b]{2}{*}{ TOTAL } & \multicolumn{4}{|c|}{ Age Group } & \multirow[b]{2}{*}{ TOTAL } & \\
\hline & $16-29$ & 30 - 39 & $40-49$ & $50+$ & & $16-29$ & 30 - 39 & $40-49$ & $50+$ & & \\
\hline Professional & 2 & 15 & 17 & 21 & 55 & 2 & 6 & 14 & 57 & 79 & 134 \\
\hline Administrative Support & 4 & 7 & 13 & 32 & 56 & 1 & 1 & 2 & 2 & 6 & 62 \\
\hline Technical Support & 0 & 2 & 4 & 11 & 17 & 4 & 2 & 7 & 15 & 28 & 45 \\
\hline Service & 0 & 0 & 0 & 1 & 1 & 0 & 0 & 3 & 1 & 4 & 5 \\
\hline Security and Fire & 1 & 0 & 1 & 1 & 3 & 18 & 7 & 18 & 12 & 55 & 58 \\
\hline Crafts & 1 & 1 & 4 & 8 & 14 & 8 & 9 & 21 & 38 & 76 & 90 \\
\hline TOTAL & 8 & 25 & 39 & 74 & 146 & 33 & 25 & 65 & 125 & 248 & 394 \\
\hline
\end{tabular}

*Only those job categories and gender/age combinations with at least one absence appear in this table.

Appendix D. Total Number of Absences by Gender, Age, and Job Category*

\begin{tabular}{|c|c|c|c|c|c|c|c|c|c|c|c|}
\hline \multirow{3}{*}{ Job Category } & \multicolumn{5}{|c|}{ Women } & \multicolumn{5}{|c|}{ Men } & \multirow[b]{3}{*}{ TOTAL } \\
\hline & \multicolumn{4}{|c|}{ Age Group } & \multirow[b]{2}{*}{ TOTAL } & \multicolumn{4}{|c|}{ Age Group } & \multirow[b]{2}{*}{ TOTAL } & \\
\hline & $16-29$ & 30 - 39 & $40-49$ & $50+$ & & $16-29$ & 30 - 39 & $40-49$ & $50+$ & & \\
\hline Professional & 3 & 23 & 20 & 21 & 67 & 2 & 6 & 15 & 63 & 86 & 153 \\
\hline Administrative Support & 6 & 9 & 16 & 38 & 69 & 1 & 1 & 3 & 2 & 7 & 76 \\
\hline Technical Support & 0 & 3 & 5 & 11 & 19 & 4 & 2 & 7 & 18 & 31 & 50 \\
\hline Service & 0 & 0 & 0 & 1 & 1 & 0 & 0 & 5 & 1 & 6 & 7 \\
\hline Security and Fire & 1 & 0 & 1 & 2 & 4 & 18 & 8 & 20 & 15 & 61 & 65 \\
\hline Crafts & 1 & 1 & 7 & 13 & 22 & 8 & 10 & 26 & 41 & 85 & 107 \\
\hline TOTAL & 11 & 36 & 49 & 86 & 182 & 33 & 27 & 76 & 140 & 276 & 458 \\
\hline
\end{tabular}

*Only those job categories and gender/age combinations with at least one absence appear in this table. 
Nevada Test Site 2006

Absence Data

Appendix E. Distribution of the Number of Calendar Days Missed per Absence by Gender and Age*

\begin{tabular}{|c|c|c|c|c|c|c|c|c|c|c|c|}
\hline \multirow{3}{*}{$\begin{array}{c}\# \\
\text { of } \\
\text { Calendar } \\
\text { Days }\end{array}$} & \multicolumn{5}{|c|}{ Women } & \multicolumn{5}{|c|}{ Men } & \multirow[b]{3}{*}{ TOTAL } \\
\hline & \multicolumn{4}{|c|}{ Age Group } & \multirow[b]{2}{*}{ TOTAL } & \multicolumn{4}{|c|}{ Age Group } & \multirow[b]{2}{*}{ TOTAL } & \\
\hline & $16-29$ & 30 - 39 & $40-49$ & $50+$ & & $16-29$ & $30-39$ & $40-49$ & $50+$ & & \\
\hline$<15$ & 10 & 19 & 26 & 38 & 93 & 28 & 17 & 48 & 77 & 170 & 263 \\
\hline $15-28$ & 1 & 5 & 9 & 21 & 36 & 1 & 5 & 15 & 21 & 42 & 78 \\
\hline $29-42$ & 0 & 5 & 8 & 9 & 22 & 1 & 1 & 2 & 17 & 21 & 43 \\
\hline $43-56$ & 0 & 2 & 3 & 7 & 12 & 1 & 0 & 1 & 7 & 9 & 21 \\
\hline $57-91$ & 0 & 3 & 3 & 6 & 12 & 0 & 4 & 7 & 13 & 24 & 36 \\
\hline $92-182$ & 0 & 2 & 0 & 4 & 6 & 2 & 0 & 3 & 5 & 10 & 16 \\
\hline $183+$ & 0 & 0 & 0 & 1 & 1 & 0 & 0 & 0 & 0 & 0 & 1 \\
\hline TOTAL & 11 & 36 & 49 & 86 & 182 & 33 & 27 & 76 & 140 & 276 & 458 \\
\hline
\end{tabular}

*Only those gender/age combinations with at least one absence appear in this table. 
Nevada Test Site 2006

Absence Data

Appendix F. Distribution of the Number of Calendar Days Missed per Absence by Gender and Job Category*

Women

\begin{tabular}{|c|c|c|c|c|c|c|c|}
\hline \multirow{2}{*}{$\begin{array}{c}\# \\
\text { of } \\
\text { Calendar } \\
\text { Days }\end{array}$} & \multicolumn{6}{|c|}{ Job Category } & \multirow[b]{2}{*}{ TOTAL } \\
\hline & Professional & $\begin{array}{c}\text { Administrative } \\
\text { Support }\end{array}$ & $\begin{array}{l}\text { Technical } \\
\text { Support }\end{array}$ & Service & $\begin{array}{c}\text { Security } \\
\text { and } \\
\text { Fire }\end{array}$ & Crafts & \\
\hline$<15$ & 32 & 37 & 8 & 0 & 2 & 14 & 93 \\
\hline $15-28$ & 14 & 12 & 6 & 1 & 1 & 2 & 36 \\
\hline $29-42$ & 12 & 7 & 0 & 0 & 1 & 2 & 22 \\
\hline $43-56$ & 5 & 4 & 1 & 0 & 0 & 2 & 12 \\
\hline $57-91$ & 3 & 5 & 2 & 0 & 0 & 2 & 12 \\
\hline $92-182$ & 1 & 4 & 1 & 0 & 0 & 0 & 6 \\
\hline $183+$ & 0 & 0 & 1 & 0 & 0 & 0 & 1 \\
\hline TOTAL & 67 & 69 & 19 & 1 & 4 & 22 & 182 \\
\hline
\end{tabular}

Men

\begin{tabular}{|c|c|c|c|c|c|c|c|}
\hline \multirow{2}{*}{$\begin{array}{c}\# \\
\text { of } \\
\text { Calendar } \\
\text { Days }\end{array}$} & \multicolumn{6}{|c|}{ Job Category } & \multirow[b]{2}{*}{ TOTAL } \\
\hline & Professional & $\begin{array}{c}\text { Administrative } \\
\text { Support }\end{array}$ & $\begin{array}{l}\text { Technical } \\
\text { Support }\end{array}$ & Service & $\begin{array}{c}\text { Security } \\
\text { and } \\
\text { Fire }\end{array}$ & Crafts & \\
\hline$<15$ & 40 & 6 & 21 & 4 & 46 & 53 & 170 \\
\hline $15-28$ & 17 & 0 & 6 & 2 & 7 & 10 & 42 \\
\hline $29-42$ & 11 & 0 & 2 & 0 & 3 & 5 & 21 \\
\hline $43-56$ & 6 & 0 & 1 & 0 & 0 & 2 & 9 \\
\hline $57-91$ & 9 & 1 & 1 & 0 & 2 & 11 & 24 \\
\hline $92-182$ & 3 & 0 & 0 & 0 & 3 & 4 & 10 \\
\hline $183+$ & 0 & 0 & 0 & 0 & 0 & 0 & 0 \\
\hline TOTAL & 86 & 7 & 31 & 6 & 61 & 85 & 276 \\
\hline
\end{tabular}

*Only those gender/job category combinations with at least one absence appear in this table. 


\section{Nevada Test Site 2006}

\section{Absence Data}

Appendix G. Number of Diagnoses in Each Diagnostic Category by Gender and Age*

\begin{tabular}{|c|c|c|c|c|c|c|}
\hline & & \multicolumn{5}{|c|}{ Women } \\
\hline & & \multicolumn{4}{|c|}{ Age Group } & \multirow[b]{2}{*}{ TOTAL } \\
\hline & & $16-29$ & 30 - 39 & $40-49$ & $\mathbf{5 0 +}$ & \\
\hline Diagnostic Category & ICD-9-CM Code & & & & & \\
\hline INFECTIOUS \& PARASITIC DISEASES (DIS) & 001-139 & 2 & 2 & 2 & 4 & 10 \\
\hline -Intestinal Infectious Dis & 001-009 & 0 & 0 & 0 & 0 & 0 \\
\hline -Other Bacterial Dis & 030-041 & 1 & 0 & 1 & 0 & 2 \\
\hline$-\mathrm{HIV}$ & 042 & 0 & 0 & 0 & 1 & 1 \\
\hline -Viral Dis with Exanthem & 050-057 & 0 & 0 & 0 & 2 & 2 \\
\hline -Other Viral Dis \& Chlamydiae & 070-079 & 1 & 2 & 1 & 1 & 5 \\
\hline -Mycoses & 110-118 & 0 & 0 & 0 & 0 & 0 \\
\hline MALIGNANT NEOPLASMS & 140-208, 230-234 & 0 & 0 & 1 & 3 & 4 \\
\hline -Digestive \& Peritoneal & $150-159$ & 0 & 0 & 0 & 0 & 0 \\
\hline -Bone, Connective Tissue, Skin & $170-173,176$ & 0 & 0 & 0 & 0 & 0 \\
\hline -Breast & 174-175 & 0 & 0 & 1 & 1 & 2 \\
\hline -Genitourinary & 179-189 & 0 & 0 & 0 & 0 & 0 \\
\hline -Other \& Unspecified Sites & 190, 193-199 & 0 & 0 & 0 & 1 & 1 \\
\hline -Lymphatic \& Hematopoietic & $200-208$ & 0 & 0 & 0 & 1 & 1 \\
\hline BENIGN \& UNCERTAIN NEOPLASMS & 210-229, 235-239 & 0 & 2 & 7 & 1 & 10 \\
\hline ENDOCRINE/METABOLIC/IMMUNITY & $240-279$ & 0 & 3 & 5 & 5 & 13 \\
\hline -Thyroid Gland Disorders & $240-246$ & 0 & 0 & 0 & 0 & 0 \\
\hline -Other Endocrine Gland Dis & $250-259$ & 0 & 0 & 3 & 2 & 5 \\
\hline -Other Metabolic \& Immunity Disorders & $270-279$ & 0 & 3 & 2 & 3 & 8 \\
\hline BLOOD \& BLOOD-FORMING ORGANS & $280-289$ & 1 & 0 & 0 & 1 & 2 \\
\hline MENTAL DISORDERS & 290-319 & 0 & 3 & 6 & 9 & 18 \\
\hline -Psychoses & 290-299 & 0 & 0 & 2 & 0 & 2 \\
\hline -Non-Psychotic Disorders & 300-302, 306-316 & 0 & 3 & 4 & 8 & 15 \\
\hline -Alcohol Dependence & 303 & 0 & 0 & 0 & 0 & 0 \\
\hline -Drug Dependence & $304-305$ & 0 & 0 & 0 & 1 & 1 \\
\hline NERVOUS SYSTEM (NS) \& SENSE ORGANS & $320-389$ & 0 & 4 & 4 & 10 & 18 \\
\hline -Hereditary/Degenerative Central NS Dis & 330-337 & 0 & 0 & 0 & 1 & 1 \\
\hline -Other Disorders of Central NS & $340-349$ & 0 & 0 & 0 & 1 & 1 \\
\hline -Disorders of Peripheral NS & $350-359$ & 0 & 3 & 0 & 5 & 8 \\
\hline -Disorders of Eye & $360-379$ & 0 & 1 & 3 & 1 & 5 \\
\hline -Dis of Ear \& Mastoid & $380-389$ & 0 & 0 & 1 & 2 & 3 \\
\hline CIRCULATORY SYSTEM & $390-459$ & 0 & 0 & 7 & 10 & 17 \\
\hline -Chronic Rheumatic Heart Dis & 393-398 & 0 & 0 & 0 & 0 & 0 \\
\hline -Hypertensive Dis & $401-405$ & 0 & 0 & 5 & 8 & 13 \\
\hline -Ischemic Heart Dis & $410-414$ & 0 & 0 & 1 & 1 & 2 \\
\hline -Other Heart Dis & $420-429$ & 0 & 0 & 0 & 0 & 0 \\
\hline -Cerebrovascular Dis & $430-438$ & 0 & 0 & 0 & 0 & 0 \\
\hline
\end{tabular}

(Continued)

*Only those diagnostic categories and gender/age combinations with at least one occurrence appear in this table. 


\section{Nevada Test Site 2006}

\section{Absence Data}

Appendix G. Number of Diagnoses in Each Diagnostic Category by Gender and Age*

\begin{tabular}{|c|c|c|c|c|c|c|c|}
\hline & & \multicolumn{5}{|c|}{ Men } & \multirow[b]{3}{*}{ TOTAL } \\
\hline & & \multicolumn{4}{|c|}{ Age Group } & \multirow[b]{2}{*}{ TOTAL } & \\
\hline & & $16-29$ & 30 - 39 & $40-49$ & $50+$ & & \\
\hline Diagnostic Category & ICD-9-CM Code & & & & & & \\
\hline INFECTIOUS \& PARASITIC DISEASES (DIS) & 001-139 & 4 & 1 & 5 & 5 & 15 & 25 \\
\hline -Intestinal Infectious Dis & 001-009 & 1 & 0 & 0 & 1 & 2 & 2 \\
\hline -Other Bacterial Dis & $030-041$ & 1 & 0 & 0 & 0 & 1 & 3 \\
\hline$-\mathrm{HIV}$ & 042 & 0 & 0 & 0 & 0 & 0 & 1 \\
\hline -Viral Dis with Exanthem & $050-057$ & 1 & 0 & 0 & 0 & 1 & 3 \\
\hline -Other Viral Dis \& Chlamydiae & 070-079 & 1 & 1 & 4 & 4 & 10 & 15 \\
\hline -Mycoses & 110-118 & 0 & 0 & 1 & 0 & 1 & 1 \\
\hline MALIGNANT NEOPLASMS & 140-208, 230-234 & 0 & 0 & 0 & 9 & 9 & 13 \\
\hline -Digestive \& Peritoneal & 150-159 & 0 & 0 & 0 & 2 & 2 & 2 \\
\hline -Bone, Connective Tissue, Skin & $170-173,176$ & 0 & 0 & 0 & 1 & 1 & 1 \\
\hline -Breast & 174-175 & 0 & 0 & 0 & 0 & 0 & 2 \\
\hline -Genitourinary & 179-189 & 0 & 0 & 0 & 4 & 4 & 4 \\
\hline -Other \& Unspecified Sites & 190, 193-199 & 0 & 0 & 0 & 1 & 1 & 2 \\
\hline -Lymphatic \& Hematopoietic & $200-208$ & 0 & 0 & 0 & 1 & 1 & 2 \\
\hline BENIGN \& UNCERTAIN NEOPLASMS & 210-229, 235-239 & 0 & 1 & 2 & 0 & 3 & 13 \\
\hline ENDOCRINE/METABOLIC/IMMUNITY & $240-279$ & 0 & 1 & 3 & 15 & 19 & 32 \\
\hline -Thyroid Gland Disorders & $240-246$ & 0 & 0 & 0 & 2 & 2 & 2 \\
\hline -Other Endocrine Gland Dis & $250-259$ & 0 & 0 & 0 & 8 & 8 & 13 \\
\hline -Other Metabolic \& Immunity Disorders & $270-279$ & 0 & 1 & 3 & 5 & 9 & 17 \\
\hline BLOOD \& BLOOD-FORMING ORGANS & $280-289$ & 0 & 0 & 0 & 2 & 2 & 4 \\
\hline MENTAL DISORDERS & $290-319$ & 0 & 1 & 12 & 3 & 16 & 34 \\
\hline -Psychoses & 290-299 & 0 & 0 & 1 & 0 & 1 & 3 \\
\hline -Non-Psychotic Disorders & $300-302,306-316$ & 0 & 1 & 9 & 2 & 12 & 27 \\
\hline -Alcohol Dependence & 303 & 0 & 0 & 1 & 1 & 2 & 2 \\
\hline -Drug Dependence & $304-305$ & 0 & 0 & 1 & 0 & 1 & 2 \\
\hline NERVOUS SYSTEM (NS) \& SENSE ORGANS & $320-389$ & 1 & 5 & 9 & 22 & 37 & 55 \\
\hline -Hereditary/Degenerative Central NS Dis & 330-337 & 0 & 0 & 0 & 0 & 0 & 1 \\
\hline -Other Disorders of Central NS & $340-349$ & 0 & 0 & 1 & 3 & 4 & 5 \\
\hline -Disorders of Peripheral NS & $350-359$ & 0 & 1 & 3 & 6 & 10 & 18 \\
\hline -Disorders of Eye & $360-379$ & 0 & 0 & 5 & 11 & 16 & 21 \\
\hline -Dis of Ear \& Mastoid & $380-389$ & 1 & 4 & 0 & 2 & 7 & 10 \\
\hline CIRCULATORY SYSTEM & $390-459$ & 1 & 2 & 15 & 29 & 47 & 64 \\
\hline -Chronic Rheumatic Heart Dis & 393-398 & 0 & 0 & 1 & 0 & 1 & 1 \\
\hline -Hypertensive Dis & $401-405$ & 1 & 0 & 6 & 8 & 15 & 28 \\
\hline -Ischemic Heart Dis & $410-414$ & 0 & 0 & 2 & 10 & 12 & 14 \\
\hline -Other Heart Dis & $420-429$ & 0 & 0 & 2 & 4 & 6 & 6 \\
\hline -Cerebrovascular Dis & $430-438$ & 0 & 0 & 0 & 2 & 2 & 2 \\
\hline
\end{tabular}

(Continued)

*Only those diagnostic categories and gender/age combinations with at least one occurrence appear in this table. 


\section{Nevada Test Site 2006}

\section{Absence Data}

Appendix G. Number of Diagnoses in Each Diagnostic Category by Gender and Age*

\begin{tabular}{|c|c|c|c|c|c|c|}
\hline & & \multicolumn{5}{|c|}{ Women } \\
\hline & & \multicolumn{4}{|c|}{ Age Group } & \multirow[b]{2}{*}{ TOTAL } \\
\hline & & $16-29$ & $30-39$ & $40-49$ & $50+$ & \\
\hline Diagnostic Category & $\begin{array}{l}\text { ICD-9-CM } \\
\text { Code }\end{array}$ & & & & & \\
\hline -Dis of Arteries \& Capillaries & 440-448 & 0 & 0 & 0 & 0 & 0 \\
\hline -Dis of Veins, Lymphatics, Other & $451-459$ & 0 & 0 & 1 & 1 & 2 \\
\hline RESPIRATORY SYSTEM & $460-519$ & 4 & 9 & 5 & 15 & 33 \\
\hline -Acute Respiratory Infections & $460-466$ & 4 & 1 & 3 & 2 & 10 \\
\hline -Other Dis Upper Respiratory Tract & $470-478$ & 0 & 3 & 1 & 6 & 10 \\
\hline -Pneumonia \& Influenza & $480-487$ & 0 & 0 & 1 & 1 & 2 \\
\hline -Chronic Obstructive Dis & $490-496$ & 0 & 1 & 0 & 5 & 6 \\
\hline -Lung Dis from External Agents & $500-508$ & 0 & 0 & 0 & 0 & 0 \\
\hline -Other Respiratory Dis & $510-519$ & 0 & 4 & 0 & 1 & 5 \\
\hline DIGESTIVE SYSTEM & $520-579$ & 1 & 3 & 6 & 14 & 24 \\
\hline -Oral Cavity, Saliva Glands, Jaw & $520-529$ & 0 & 0 & 0 & 0 & 0 \\
\hline -Esophagus, Stomach, Duodenum & $530-537$ & 0 & 0 & 0 & 1 & 1 \\
\hline -Appendicitis & $540-543$ & 0 & 0 & 2 & 0 & 2 \\
\hline -Hernia & $550-553$ & 0 & 0 & 0 & 1 & 1 \\
\hline -Enteritis, Colitis & $555-558$ & 0 & 1 & 0 & 3 & 4 \\
\hline -Other Intestinal Dis & $560-569$ & 1 & 1 & 1 & 2 & 5 \\
\hline -Other Digestive Dis & $570-579$ & 0 & 1 & 3 & 7 & 11 \\
\hline GENITOURINARY SYSTEM & $580-629$ & 5 & 12 & 4 & 10 & 31 \\
\hline -Nephritis, Nephrosis & $580-589$ & 0 & 0 & 0 & 0 & 0 \\
\hline -Other Urinary Dis & $590-599$ & 2 & 0 & 3 & 1 & 6 \\
\hline -Male Genital Organ Dis & $600-608$ & 0 & 0 & 0 & 0 & 0 \\
\hline -Other Female Disorders & $617-629$ & 3 & 12 & 1 & 9 & 25 \\
\hline SKIN \& SUBCUTANEOUS TISSUE & 680-709 & 0 & 1 & 1 & 3 & 5 \\
\hline -Infections & $680-686$ & 0 & 1 & 0 & 0 & 1 \\
\hline -Other Inflammatory Conditions & $690-698$ & 0 & 0 & 0 & 3 & 3 \\
\hline -Other & 700-709 & 0 & 0 & 1 & 0 & 1 \\
\hline MUSCULOSKELETAL \& CONNECTIVE TISSUE & 710-739 & 1 & 23 & 12 & 26 & 62 \\
\hline -Arthropathies & $710-719$ & 0 & 2 & 4 & 13 & 19 \\
\hline -Dorsopathies & $720-724$ & 1 & 17 & 3 & 8 & 29 \\
\hline -Rheumatism, Excluding Back & 725-729 & 0 & 3 & 4 & 4 & 11 \\
\hline -Other Dis \& Acquired Deformities & $730-739$ & 0 & 1 & 1 & 1 & 3 \\
\hline CONGENITAL ANOMALIES & $740-759$ & 0 & 0 & 0 & 0 & 0 \\
\hline SYMPTOMS, SIGNS, \& ILL-DEFINED CONDITIONS & 780-799 & 1 & 8 & 10 & 38 & 57 \\
\hline -Symptoms & 780-789 & 1 & 8 & 9 & 36 & 54 \\
\hline -Non-Specific Abnormal Findings & $790-796$ & 0 & 0 & 1 & 2 & 3 \\
\hline -Ill-Defined \& Unknown Causes & 797-799 & 0 & 0 & 0 & 0 & 0 \\
\hline
\end{tabular}

(Continued)

*Only those diagnostic categories and gender/age combinations with at least one occurrence appear in this table. 


\section{Nevada Test Site 2006}

\section{Absence Data}

Appendix G. Number of Diagnoses in Each Diagnostic Category by Gender and Age*

\begin{tabular}{|c|c|c|c|c|c|c|c|}
\hline & & \multicolumn{5}{|c|}{ Men } & \multirow[b]{3}{*}{ TOTAL } \\
\hline & & \multicolumn{4}{|c|}{ Age Group } & \multirow[b]{2}{*}{ TOTAL } & \\
\hline & & $16-29$ & $30-39$ & $40-49$ & $50+$ & & \\
\hline Diagnostic Category & $\begin{array}{l}\text { ICD-9-CM } \\
\text { Code }\end{array}$ & & & & & & \\
\hline -Dis of Arteries \& Capillaries & $440-448$ & 0 & 0 & 1 & 4 & 5 & 5 \\
\hline -Dis of Veins, Lymphatics, Other & $451-459$ & 0 & 2 & 3 & 1 & 6 & 8 \\
\hline RESPIRATORY SYSTEM & $460-519$ & 10 & 9 & 19 & 26 & 64 & 97 \\
\hline -Acute Respiratory Infections & $460-466$ & 5 & 5 & 3 & 8 & 21 & 31 \\
\hline -Other Dis Upper Respiratory Tract & $470-478$ & 2 & 3 & 7 & 4 & 16 & 26 \\
\hline -Pneumonia \& Influenza & $480-487$ & 0 & 0 & 4 & 8 & 12 & 14 \\
\hline -Chronic Obstructive Dis & $490-496$ & 3 & 0 & 2 & 3 & 8 & 14 \\
\hline -Lung Dis from External Agents & $500-508$ & 0 & 0 & 2 & 0 & 2 & 2 \\
\hline -Other Respiratory Dis & $510-519$ & 0 & 1 & 1 & 3 & 5 & 10 \\
\hline DIGESTIVE SYSTEM & $520-579$ & 2 & 7 & 13 & 29 & 51 & 75 \\
\hline -Oral Cavity, Saliva Glands, Jaw & $520-529$ & 0 & 0 & 0 & 1 & 1 & 1 \\
\hline -Esophagus, Stomach, Duodenum & $530-537$ & 0 & 0 & 5 & 2 & 7 & 8 \\
\hline -Appendicitis & $540-543$ & 0 & 0 & 0 & 2 & 2 & 4 \\
\hline -Hernia & $550-553$ & 0 & 6 & 2 & 9 & 17 & 18 \\
\hline -Enteritis, Colitis & $555-558$ & 2 & 1 & 3 & 3 & 9 & 13 \\
\hline -Other Intestinal Dis & $560-569$ & 0 & 0 & 1 & 2 & 3 & 8 \\
\hline -Other Digestive Dis & $570-579$ & 0 & 0 & 2 & 10 & 12 & 23 \\
\hline GENITOURINARY SYSTEM & $580-629$ & 2 & 0 & 4 & 8 & 14 & 45 \\
\hline -Nephritis, Nephrosis & 580-589 & 0 & 0 & 0 & 1 & 1 & 1 \\
\hline -Other Urinary Dis & $590-599$ & 2 & 0 & 3 & 7 & 12 & 18 \\
\hline -Male Genital Organ Dis & $600-608$ & 0 & 0 & 1 & 0 & 1 & 1 \\
\hline -Other Female Disorders & 617-629 & 0 & 0 & 0 & 0 & 0 & 25 \\
\hline SKIN \& SUBCUTANEOUS TISSUE & 680-709 & 0 & 1 & 7 & 2 & 10 & 15 \\
\hline -Infections & $680-686$ & 0 & 1 & 5 & 1 & 7 & 8 \\
\hline -Other Inflammatory Conditions & $690-698$ & 0 & 0 & 1 & 1 & 2 & 5 \\
\hline -Other & 700-709 & 0 & 0 & 1 & 0 & 1 & 2 \\
\hline MUSCULOSKELETAL \& CONNECTIVE TISSUE & 710-739 & 15 & 11 & 21 & 38 & 85 & 147 \\
\hline -Arthropathies & $710-719$ & 6 & 2 & 5 & 16 & 29 & 48 \\
\hline -Dorsopathies & $720-724$ & 7 & 6 & 10 & 12 & 35 & 64 \\
\hline -Rheumatism, Excluding Back & 725-729 & 1 & 3 & 6 & 7 & 17 & 28 \\
\hline -Other Dis \& Acquired Deformities & 730-739 & 1 & 0 & 0 & 3 & 4 & 7 \\
\hline CONGENITAL ANOMALIES & $740-759$ & 0 & 0 & 1 & 0 & 1 & 1 \\
\hline SYMPTOMS, SIGNS, \& ILL-DEFINED CONDITIONS & 780-799 & 13 & 7 & 33 & 28 & 81 & 138 \\
\hline -Symptoms & 780-789 & 12 & 7 & 33 & 26 & 78 & 132 \\
\hline -Non-Specific Abnormal Findings & $790-796$ & 1 & 0 & 0 & 1 & 2 & 5 \\
\hline -Ill-Defined \& Unknown Causes & 797-799 & 0 & 0 & 0 & 1 & 1 & 1 \\
\hline
\end{tabular}

(Continued)

*Only those diagnostic categories and gender/age combinations with at least one occurrence appear in this table. 


\section{Nevada Test Site 2006}

\section{Absence Data}

Appendix G. Number of Diagnoses in Each Diagnostic Category by Gender and Age*

\begin{tabular}{|c|c|c|c|c|c|c|}
\hline & & \multicolumn{5}{|c|}{ Women } \\
\hline & & \multicolumn{4}{|c|}{ Age Group } & \multirow[b]{2}{*}{ TOTAL } \\
\hline & & $16-29$ & $30-39$ & 40 - 49 & $50+$ & \\
\hline Diagnostic Category & $\begin{array}{l}\text { ICD-9-CM } \\
\text { Code }\end{array}$ & & & & & \\
\hline INJURY \& POISONING & $800-999$ & 1 & 6 & 9 & 15 & 31 \\
\hline -Fracture - Skull & $800-804$ & 0 & 0 & 0 & 1 & 1 \\
\hline -Fracture - Neck, Trunk & 805-809 & 0 & 0 & 0 & 0 & 0 \\
\hline -Fracture - Upper Limb & 810-819 & 0 & 1 & 0 & 2 & 3 \\
\hline -Fracture - Lower Limb & 820-829 & 0 & 0 & 3 & 2 & 5 \\
\hline -Dislocation & $830-839$ & 0 & 0 & 2 & 1 & 3 \\
\hline -Sprains \& Strains - Back & 846-847 & 0 & 1 & 0 & 1 & 2 \\
\hline -Sprains \& Strains - Other & $840-845,848$ & 0 & 1 & 2 & 4 & 7 \\
\hline -Intracranial Injury & $850-854$ & 0 & 0 & 1 & 0 & 1 \\
\hline -Internal Injury - Thorax, Abdomen, Pelvis & $860-869$ & 0 & 0 & 0 & 0 & 0 \\
\hline -Open Wound - Head, Neck, Trunk & 870-879 & 0 & 0 & 0 & 0 & 0 \\
\hline -Open Wound - Upper Limb & $880-887$ & 0 & 0 & 0 & 0 & 0 \\
\hline -Open Wound - Lower Limb & 890-897 & 0 & 0 & 0 & 0 & 0 \\
\hline -Superficial Injury & 910-919 & 0 & 0 & 0 & 1 & 1 \\
\hline -Contusion & 920-924 & 1 & 1 & 1 & 2 & 5 \\
\hline -Burns & 940-949 & 0 & 0 & 0 & 0 & 0 \\
\hline -Injury to Nerves \& Spinal Cord & 950-957 & 0 & 0 & 0 & 0 & 0 \\
\hline -Complications \& Unspecified Injuries & 958-959 & 0 & 0 & 0 & 0 & 0 \\
\hline -Toxic Effects - Non-medicinal & 980-989 & 0 & 0 & 0 & 0 & 0 \\
\hline -Unspecified Effects - External Causes & $990-995$ & 0 & 0 & 0 & 1 & 1 \\
\hline -Complications of Surgical/Medical Care & 996-999 & 0 & 2 & 0 & 0 & 2 \\
\hline HEALTH STATUS/HEALTH SERVICE CONTACT & V01-V82 & 0 & 1 & 1 & 5 & 7 \\
\hline -Isolation \& Prophylactic Measures & V07-V09 & 0 & 0 & 0 & 1 & 1 \\
\hline -Personal \& Family History & V10-V19 & 0 & 0 & 0 & 2 & 2 \\
\hline -Health Services Reproduction/Development & V20-V29 & 0 & 0 & 0 & 0 & 0 \\
\hline -Health Status & V40-V49 & 0 & 1 & 0 & 0 & 1 \\
\hline -Specific Procedures/Aftercare & V50-V59 & 0 & 0 & 1 & 2 & 3 \\
\hline -Other Circumstances & V60-V69 & 0 & 0 & 0 & 0 & 0 \\
\hline -Examination \& Investigation & V70-V82 & 0 & 0 & 0 & 0 & 0 \\
\hline
\end{tabular}

*Only those diagnostic categories and gender/age combinations with at least one occurrence appear in this table. 


\section{Nevada Test Site 2006}

\section{Absence Data}

Appendix G. Number of Diagnoses in Each Diagnostic Category by Gender and Age*

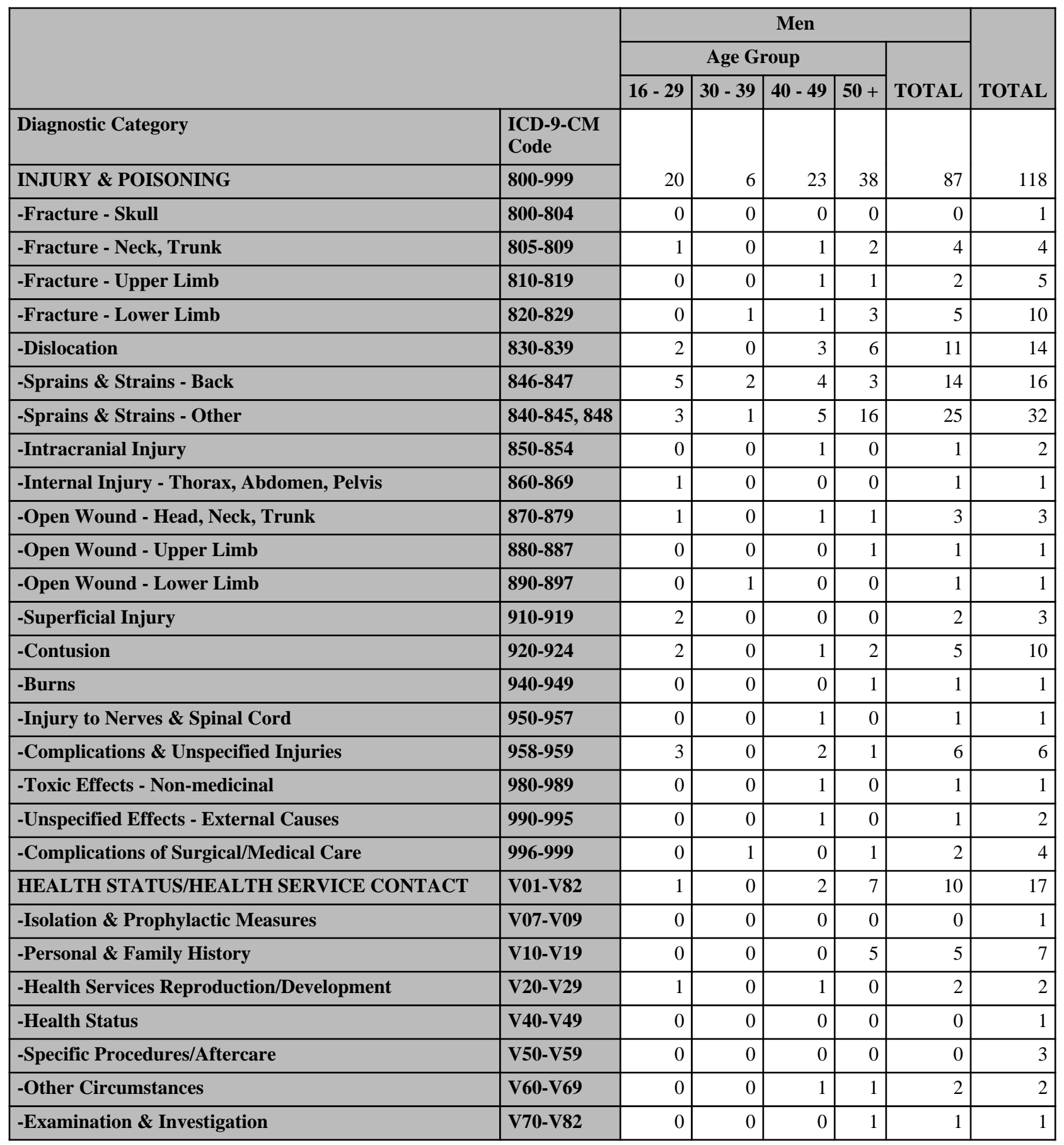

*Only those diagnostic categories and gender/age combinations with at least one occurrence appear in this table. 


\section{Nevada Test Site 2006}

Absence Data

Appendix G. Number of Diagnoses in Each Diagnostic Category by Gender and Age*

\begin{tabular}{|c|c|c|c|c|c|c|c|c|c|c|c|}
\hline & \multicolumn{5}{|c|}{ Women } & \multicolumn{5}{|c|}{ Men } & \multirow[b]{3}{*}{ TOTAL } \\
\hline & \multicolumn{4}{|c|}{ Age Group } & \multirow[b]{2}{*}{ TOTAL } & \multicolumn{4}{|c|}{ Age Group } & \multirow[b]{2}{*}{ TOTAL } & \\
\hline & $16-29$ & $30-39$ & $40-49$ & $50+$ & & $16-29$ & 30 - 39 & $40-49$ & $50+$ & & \\
\hline $\begin{array}{l}\text { Diagnostic } \\
\text { Category }\end{array}$ & & & & & & & & & & & \\
\hline Total & 16 & 77 & 80 & 169 & 342 & 69 & 52 & 169 & 261 & 551 & 893 \\
\hline
\end{tabular}

*Only those diagnostic categories and gender/age combinations with at least one occurrence appear in this table. 
Nevada Test Site 2006

Absence Data

Appendix H. Total Number of Calendar Days Absent in Each Diagnostic Category by Gender and Age*

\begin{tabular}{|c|c|c|c|c|c|c|}
\hline & & \multicolumn{5}{|c|}{ Women } \\
\hline & & \multicolumn{4}{|c|}{ Age Group } & \multirow[b]{2}{*}{ TOTAL } \\
\hline & & $16-29$ & $30-39$ & $40-49$ & $50+$ & \\
\hline Diagnostic Category & ICD-9-CM Code & \multirow[b]{2}{*}{17} & \multirow[b]{2}{*}{9} & \multirow[b]{2}{*}{18} & \multirow[b]{2}{*}{101} & \multirow[b]{2}{*}{145} \\
\hline INFECTIOUS \& PARASITIC DISEASES (DIS) & 001-139 & & & & & \\
\hline MALIGNANT NEOPLASMS & 140-208, 230-234 & 0 & 0 & 30 & 491 & 521 \\
\hline BENIGN \& UNCERTAIN NEOPLASMS & 210-229, 235-239 & 0 & 82 & 235 & 49 & 366 \\
\hline ENDOCRINE/METABOLIC/IMMUNITY & $240-279$ & 0 & 51 & 37 & 74 & 162 \\
\hline BLOOD \& BLOOD-FORMING ORGANS & $280-289$ & 11 & 0 & 0 & 8 & 19 \\
\hline MENTAL DISORDERS & $290-319$ & 0 & 172 & 189 & 307 & 668 \\
\hline NERVOUS SYSTEM (NS) \& SENSE ORGANS & $320-389$ & 0 & 131 & 71 & 235 & 437 \\
\hline CIRCULATORY SYSTEM & $390-459$ & 0 & 0 & 158 & 189 & 347 \\
\hline RESPIRATORY SYSTEM & $460-519$ & 8 & 34 & 49 & 165 & 256 \\
\hline DIGESTIVE SYSTEM & $520-579$ & 10 & 37 & 63 & 267 & 377 \\
\hline GENITOURINARY SYSTEM & $580-629$ & 49 & 244 & 87 & 416 & 796 \\
\hline SKIN \& SUBCUTANEOUS TISSUE & $680-709$ & 0 & 7 & 6 & 122 & 135 \\
\hline MUSCULOSKELETAL \& CONNECTIVE TISSUE & $710-739$ & 22 & 368 & 220 & 474 & 1,084 \\
\hline CONGENITAL ANOMALIES & $740-759$ & 0 & 0 & 0 & 0 & 0 \\
\hline SYMPTOMS, SIGNS, \& ILL-DEFINED CONDITIONS & 780-799 & 6 & 43 & 139 & 542 & 730 \\
\hline INJURY \& POISONING & $800-999$ & 7 & 88 & 153 & 379 & 627 \\
\hline
\end{tabular}

\footnotetext{
*Absences with >1 ICD-9-CM code in the same diagnostic category were counted only once. Only those diagnostic categories and gender/age combinations with at least one occurrence appear in this table.
} 


\section{Nevada Test Site 2006}

Absence Data

Appendix H. Total Number of Calendar Days Absent in Each Diagnostic Category by Gender and Age*

\begin{tabular}{|c|c|c|c|c|c|c|c|}
\hline & & \multicolumn{5}{|c|}{ Men } & \multirow[b]{3}{*}{ TOTAL } \\
\hline & & \multicolumn{4}{|c|}{ Age Group } & \multirow[b]{2}{*}{ TOTAL } & \\
\hline & & $16-29$ & $30-39$ & $40-49$ & $\mathbf{5 0 +}$ & & \\
\hline Diagnostic Category & ICD-9-CM Code & & & & & & \\
\hline INFECTIOUS \& PARASITIC DISEASES (DIS) & $001-139$ & 22 & 1 & 46 & 56 & 125 & 270 \\
\hline MALIGNANT NEOPLASMS & $140-208,230-234$ & 0 & 0 & 0 & 305 & 305 & 826 \\
\hline BENIGN \& UNCERTAIN NEOPLASMS & 210-229, 235-239 & 0 & 13 & 105 & 0 & 118 & 484 \\
\hline ENDOCRINE/METABOLIC/IMMUNITY & $240-279$ & 0 & 42 & 37 & 424 & 503 & 665 \\
\hline BLOOD \& BLOOD-FORMING ORGANS & $280-289$ & 0 & 0 & 0 & 35 & 35 & 54 \\
\hline MENTAL DISORDERS & $290-319$ & 0 & 8 & 265 & 137 & 410 & 1,078 \\
\hline NERVOUS SYSTEM (NS) \& SENSE ORGANS & $320-389$ & 4 & 30 & 41 & 269 & 344 & 781 \\
\hline CIRCULATORY SYSTEM & $390-459$ & 4 & 99 & 252 & 484 & 839 & 1,186 \\
\hline RESPIRATORY SYSTEM & $460-519$ & 43 & 27 & 155 & 244 & 469 & 725 \\
\hline DIGESTIVE SYSTEM & $520-579$ & 7 & 105 & 190 & 434 & 736 & 1,113 \\
\hline GENITOURINARY SYSTEM & $580-629$ & 4 & 0 & 28 & 124 & 156 & 952 \\
\hline SKIN \& SUBCUTANEOUS TISSUE & 680-709 & 0 & 1 & 70 & 32 & 103 & 238 \\
\hline MUSCULOSKELETAL \& CONNECTIVE TISSUE & \begin{tabular}{|l|}
$710-739$ \\
\end{tabular} & 220 & 194 & 380 & 1,441 & 2,235 & 3,319 \\
\hline CONGENITAL ANOMALIES & 740-759 & 0 & 0 & 12 & 0 & 12 & 12 \\
\hline SYMPTOMS, SIGNS, \& ILL-DEFINED CONDITIONS & 780-799 & 47 & 8 & 337 & 393 & 785 & 1,515 \\
\hline INJURY \& POISONING & \begin{tabular}{|l|}
$800-999$ \\
\end{tabular} & 385 & 68 & 611 & 998 & 2,062 & 2,689 \\
\hline
\end{tabular}

\footnotetext{
*Absences with >1 ICD-9-CM code in the same diagnostic category were counted only once. Only those diagnostic categories and gender/age combinations with at least one occurrence appear in this table.
} 


\section{Nevada Test Site 2006}

\section{Absence Data}

Appendix I. Number of Diagnoses in Each Diagnostic Category by Gender and Job Category*

\begin{tabular}{|c|c|c|c|c|c|c|c|c|}
\hline & & \multicolumn{7}{|c|}{ Women } \\
\hline & & \multicolumn{6}{|c|}{ Job Category } & \multirow[b]{2}{*}{ TOTAL } \\
\hline & & Professional & $\begin{array}{l}\text { Administrative } \\
\text { Support }\end{array}$ & $\begin{array}{c}\text { Technical } \\
\text { Support }\end{array}$ & Service & $\begin{array}{c}\text { Security } \\
\text { and } \\
\text { Fire }\end{array}$ & Crafts & \\
\hline Diagnostic Category & $\begin{array}{l}\text { ICD-9-CM } \\
\text { Code }\end{array}$ & & & & & & & \\
\hline $\begin{array}{l}\text { INFECTIOUS \& PARASITIC DISEASES } \\
\text { (DIS) }\end{array}$ & 001-139 & 2 & 5 & 1 & 0 & 0 & 2 & 10 \\
\hline -Other Bacterial Dis & 030-041 & 1 & 1 & 0 & 0 & 0 & 0 & 2 \\
\hline -HIV & 042 & 0 & 1 & 0 & 0 & 0 & 0 & 1 \\
\hline -Viral Dis with Exanthem & $050-057$ & 0 & 2 & 0 & 0 & 0 & 0 & 2 \\
\hline -Other Viral Dis \& Chlamydiae & 070-079 & 1 & 1 & 1 & 0 & 0 & 2 & 5 \\
\hline MALIGNANT NEOPLASMS & $\begin{array}{l}140-208 \\
230-234\end{array}$ & 2 & 0 & 2 & 0 & 0 & 0 & 4 \\
\hline -Breast & 174-175 & 1 & 0 & 1 & 0 & 0 & 0 & 2 \\
\hline -Other \& Unspecified Sites & $\begin{array}{l}190 \\
193-199\end{array}$ & 1 & 0 & 0 & 0 & 0 & 0 & 1 \\
\hline -Lymphatic \& Hematopoietic & 200-208 & 0 & 0 & 1 & 0 & 0 & 0 & 1 \\
\hline BENIGN \& UNCERTAIN NEOPLASMS & $\begin{array}{l}\text { 210-229, } \\
235-239\end{array}$ & 5 & 4 & 0 & 0 & 0 & 1 & 10 \\
\hline ENDOCRINE/METABOLIC/IMMUNITY & $240-279$ & 6 & 7 & 0 & 0 & 0 & 0 & 13 \\
\hline -Other Endocrine Gland Dis & $250-259$ & 1 & 4 & 0 & 0 & 0 & 0 & 5 \\
\hline -Other Metabolic \& Immunity Disorders & $270-279$ & 5 & 3 & 0 & 0 & 0 & 0 & 8 \\
\hline BLOOD \& BLOOD-FORMING ORGANS & 280-289 & 2 & 0 & 0 & 0 & 0 & 0 & 2 \\
\hline MENTAL DISORDERS & 290-319 & 7 & 6 & 1 & 0 & 0 & 4 & 18 \\
\hline -Psychoses & 290-299 & 2 & 0 & 0 & 0 & 0 & 0 & 2 \\
\hline -Non-Psychotic Disorders & $\begin{array}{l}300-302 \\
306-316\end{array}$ & 5 & 5 & 1 & 0 & 0 & 4 & 15 \\
\hline -Drug Dependence & $304-305$ & 0 & 1 & 0 & 0 & 0 & 0 & 1 \\
\hline $\begin{array}{l}\text { NERVOUS SYSTEM (NS) \& SENSE } \\
\text { ORGANS }\end{array}$ & $320-389$ & 9 & 7 & 0 & 0 & 1 & 1 & 18 \\
\hline -Hereditary/Degenerative Central NS Dis & 330-337 & 1 & 0 & 0 & 0 & 0 & 0 & 1 \\
\hline -Other Disorders of Central NS & $340-349$ & 0 & 0 & 0 & 0 & 1 & 0 & 1 \\
\hline -Disorders of Peripheral NS & $350-359$ & 5 & 3 & 0 & 0 & 0 & 0 & 8 \\
\hline -Disorders of Eye & $360-379$ & 3 & 1 & 0 & 0 & 0 & 1 & 5 \\
\hline -Dis of Ear \& Mastoid & 380-389 & 0 & 3 & 0 & 0 & 0 & 0 & 3 \\
\hline CIRCULATORY SYSTEM & $390-459$ & 4 & 8 & 1 & 0 & 1 & 3 & 17 \\
\hline -Hypertensive Dis & $401-405$ & 3 & 6 & 1 & 0 & 1 & 2 & 13 \\
\hline -Ischemic Heart Dis & $410-414$ & 1 & 1 & 0 & 0 & 0 & 0 & 2 \\
\hline -Dis of Veins, Lymphatics, Other & 451-459 & 0 & 1 & 0 & 0 & 0 & 1 & 2 \\
\hline RESPIRATORY SYSTEM & $460-519$ & 10 & 13 & 2 & 1 & 3 & 4 & 33 \\
\hline -Acute Respiratory Infections & $460-466$ & 2 & 2 & 1 & 0 & 3 & 2 & 10 \\
\hline -Other Dis Upper Respiratory Tract & $470-478$ & 2 & 7 & 0 & 0 & 0 & 1 & 10 \\
\hline
\end{tabular}

(Continued)

*Only those diagnostic categories and gender/job category combinations with at least one occurrence appear in this table. 


\section{Nevada Test Site 2006}

\section{Absence Data}

Appendix I. Number of Diagnoses in Each Diagnostic Category by Gender and Job Category*

\begin{tabular}{|c|c|c|c|c|c|c|c|c|}
\hline & & \multicolumn{7}{|c|}{ Women } \\
\hline & & \multicolumn{6}{|c|}{ Job Category } & \multirow[b]{2}{*}{ TOTAL } \\
\hline & & Professional & $\begin{array}{l}\text { Administrative } \\
\text { Support }\end{array}$ & $\begin{array}{l}\text { Technical } \\
\text { Support }\end{array}$ & Service & $\begin{array}{c}\text { Security } \\
\text { and } \\
\text { Fire }\end{array}$ & Crafts & \\
\hline Diagnostic Category & $\begin{array}{l}\text { ICD-9-CM } \\
\text { Code }\end{array}$ & & & & & & & \\
\hline -Pneumonia \& Influenza & $480-487$ & 0 & 0 & 1 & 1 & 0 & 0 & 2 \\
\hline -Chronic Obstructive Dis & $490-496$ & 1 & 4 & 0 & 0 & 0 & 1 & 6 \\
\hline -Other Respiratory Dis & $510-519$ & 5 & 0 & 0 & 0 & 0 & 0 & 5 \\
\hline DIGESTIVE SYSTEM & \begin{tabular}{|l|}
$520-579$ \\
\end{tabular} & 12 & 6 & 4 & 0 & 0 & 2 & 24 \\
\hline -Esophagus, Stomach, Duodenum & $530-537$ & 1 & 0 & 0 & 0 & 0 & 0 & 1 \\
\hline -Appendicitis & $540-543$ & 2 & 0 & 0 & 0 & 0 & 0 & 2 \\
\hline -Hernia & $550-553$ & 0 & 0 & 0 & 0 & 0 & 1 & 1 \\
\hline -Enteritis, Colitis & $555-558$ & 1 & 2 & 0 & 0 & 0 & 1 & 4 \\
\hline -Other Intestinal Dis & $560-569$ & 3 & 2 & 0 & 0 & 0 & 0 & 5 \\
\hline -Other Digestive Dis & \begin{tabular}{|l}
$570-579$ \\
\end{tabular} & 5 & 2 & 4 & 0 & 0 & 0 & 11 \\
\hline GENITOURINARY SYSTEM & $580-629$ & 13 & 16 & 1 & 0 & 0 & 1 & 31 \\
\hline -Other Urinary Dis & $590-599$ & 0 & 6 & 0 & 0 & 0 & 0 & 6 \\
\hline -Other Female Disorders & $617-629$ & 13 & 10 & 1 & 0 & 0 & 1 & 25 \\
\hline SKIN \& SUBCUTANEOUS TISSUE & $680-709$ & 0 & 4 & 1 & 0 & 0 & 0 & 5 \\
\hline -Infections & $680-686$ & 0 & 1 & 0 & 0 & 0 & 0 & 1 \\
\hline -Other Inflammatory Conditions & $690-698$ & 0 & 2 & 1 & 0 & 0 & 0 & 3 \\
\hline -Other & $700-709$ & 0 & 1 & 0 & 0 & 0 & 0 & 1 \\
\hline $\begin{array}{l}\text { MUSCULOSKELETAL \& CONNECTIVE } \\
\text { TISSUE }\end{array}$ & $710-739$ & 31 & 15 & 4 & 0 & 0 & 12 & 62 \\
\hline -Arthropathies & $710-719$ & 7 & 7 & 0 & 0 & 0 & 5 & 19 \\
\hline -Dorsopathies & $720-724$ & 16 & 4 & 4 & 0 & 0 & 5 & 29 \\
\hline -Rheumatism, Excluding Back & $725-729$ & 5 & 4 & 0 & 0 & 0 & 2 & 11 \\
\hline -Other Dis \& Acquired Deformities & 730-739 & 3 & 0 & 0 & 0 & 0 & 0 & 3 \\
\hline $\begin{array}{l}\text { SYMPTOMS, SIGNS, \& ILL-DEFINED } \\
\text { CONDITIONS }\end{array}$ & 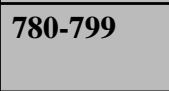 & 20 & 21 & 8 & 0 & 1 & 7 & 57 \\
\hline -Symptoms & 780-789 & 20 & 20 & 7 & 0 & 0 & 7 & 54 \\
\hline -Non-Specific Abnormal Findings & $790-796$ & 0 & 1 & 1 & 0 & 1 & 0 & 3 \\
\hline INJURY \& POISONING & $800-999$ & 9 & 9 & 2 & 0 & 0 & 11 & 31 \\
\hline -Fracture - Skull & 800-804 & 0 & 0 & 1 & 0 & 0 & 0 & 1 \\
\hline -Fracture - Upper Limb & $810-819$ & 1 & 1 & 0 & 0 & 0 & 1 & 3 \\
\hline -Fracture - Lower Limb & $820-829$ & 0 & 5 & 0 & 0 & 0 & 0 & 5 \\
\hline -Dislocation & 830-839 & 2 & 0 & 0 & 0 & 0 & 1 & 3 \\
\hline -Sprains \& Strains - Back & $846-847$ & 1 & 0 & 0 & 0 & 0 & 1 & 2 \\
\hline -Sprains \& Strains - Other & $\begin{array}{l}840-845 \\
848\end{array}$ & 1 & 1 & 1 & 0 & 0 & 4 & 7 \\
\hline -Intracranial Injury & $850-854$ & 1 & 0 & 0 & 0 & 0 & 0 & 1 \\
\hline
\end{tabular}

(Continued)

*Only those diagnostic categories and gender/job category combinations with at least one occurrence appear in this table. 


\section{Nevada Test Site 2006}

Absence Data

Appendix I. Number of Diagnoses in Each Diagnostic Category by Gender and Job Category*

\begin{tabular}{|c|c|c|c|c|c|c|c|c|}
\hline & & \multicolumn{7}{|c|}{ Women } \\
\hline & & \multicolumn{6}{|c|}{ Job Category } & \multirow[b]{2}{*}{ TOTAL } \\
\hline & & Professional & $\begin{array}{l}\text { Administrative } \\
\text { Support }\end{array}$ & $\begin{array}{l}\text { Technical } \\
\text { Support }\end{array}$ & Service & $\begin{array}{c}\text { Security } \\
\text { and } \\
\text { Fire }\end{array}$ & Crafts & \\
\hline Diagnostic Category & $\begin{array}{l}\text { ICD-9-CM } \\
\text { Code }\end{array}$ & \multirow[b]{2}{*}{0} & \multirow[b]{2}{*}{0} & \multirow[b]{2}{*}{0} & \multirow[b]{2}{*}{0} & \multirow[b]{2}{*}{0} & \multirow[b]{2}{*}{1} & \multirow[b]{2}{*}{1} \\
\hline -Superficial Injury & 910-919 & & & & & & & \\
\hline -Contusion & $920-924$ & 1 & 1 & 0 & 0 & 0 & 3 & 5 \\
\hline -Unspecified Effects - External Causes & $990-995$ & 0 & 1 & 0 & 0 & 0 & 0 & 1 \\
\hline -Complications of Surgical/Medical Care & 996-999 & 2 & 0 & 0 & 0 & 0 & 0 & 2 \\
\hline $\begin{array}{l}\text { HEALTH STATUS/HEALTH SERVICE } \\
\text { CONTACT }\end{array}$ & V01-V82 & 2 & 4 & 1 & 0 & 0 & 0 & 7 \\
\hline -Isolation \& Prophylactic Measures & V07-V09 & 0 & 1 & 0 & 0 & 0 & 0 & 1 \\
\hline -Personal \& Family History & V10-V19 & 1 & 1 & 0 & 0 & 0 & 0 & 2 \\
\hline -Health Status & V40-V49 & 1 & 0 & 0 & 0 & 0 & 0 & 1 \\
\hline -Specific Procedures/Aftercare & V50-V59 & 0 & 2 & 1 & 0 & 0 & 0 & 3 \\
\hline
\end{tabular}

\begin{tabular}{|c|c|c|c|c|c|c|c|}
\hline & \multicolumn{7}{|c|}{ Women } \\
\hline & \multicolumn{6}{|c|}{ Job Category } & \multirow[b]{2}{*}{ TOTAL } \\
\hline & Professional & $\begin{array}{c}\text { Administrative } \\
\text { Support }\end{array}$ & $\begin{array}{l}\text { Technical } \\
\text { Support }\end{array}$ & Service & $\begin{array}{l}\text { Security } \\
\text { and } \\
\text { Fire }\end{array}$ & Crafts & \\
\hline $\begin{array}{l}\text { Diagnostic } \\
\text { Category }\end{array}$ & & & & & & & \\
\hline Total & 134 & 125 & 28 & 1 & 6 & 48 & 342 \\
\hline
\end{tabular}

*Only those diagnostic categories and gender/job category combinations with at least one occurrence appear in this table. 


\section{Nevada Test Site 2006}

\section{Absence Data}

Appendix I. Number of Diagnoses in Each Diagnostic Category by Gender and Job Category*

\begin{tabular}{|c|c|c|c|c|c|c|c|c|}
\hline & & \multicolumn{7}{|c|}{ Men } \\
\hline & & \multicolumn{6}{|c|}{ Job Category } & \multirow[b]{2}{*}{ TOTAL } \\
\hline & & Professional & $\begin{array}{c}\text { Administrative } \\
\text { Support }\end{array}$ & $\begin{array}{l}\text { Technical } \\
\text { Support }\end{array}$ & Service & $\begin{array}{c}\text { Security } \\
\text { and } \\
\text { Fire }\end{array}$ & Crafts & \\
\hline Diagnostic Category & \begin{tabular}{|l|} 
ICD-9-CM \\
Code
\end{tabular} & & & & & & & \\
\hline $\begin{array}{l}\text { INFECTIOUS \& PARASITIC DISEASES } \\
\text { (DIS) }\end{array}$ & 001-139 & 5 & 0 & 2 & 0 & 3 & 5 & 15 \\
\hline -Intestinal Infectious Dis & 001-009 & 1 & 0 & 1 & 0 & 0 & 0 & 2 \\
\hline -Other Bacterial Dis & 030-041 & 1 & 0 & 0 & 0 & 0 & 0 & 1 \\
\hline -Viral Dis with Exanthem & $050-057$ & 0 & 0 & 0 & 0 & 0 & 1 & 1 \\
\hline -Other Viral Dis \& Chlamydiae & 070-079 & 2 & 0 & 1 & 0 & 3 & 4 & 10 \\
\hline -Mycoses & 110-118 & 1 & 0 & 0 & 0 & 0 & 0 & 1 \\
\hline MALIGNANT NEOPLASMS & $\begin{array}{l}140-208 \\
230-234\end{array}$ & 6 & 0 & 0 & 0 & 2 & 1 & 9 \\
\hline -Digestive \& Peritoneal & $150-159$ & 1 & 0 & 0 & 0 & 0 & 1 & 2 \\
\hline -Bone, Connective Tissue, Skin & $\begin{array}{l}170-173 \\
176\end{array}$ & 0 & 0 & 0 & 0 & 1 & 0 & 1 \\
\hline -Genitourinary & 179-189 & 4 & 0 & 0 & 0 & 0 & 0 & 4 \\
\hline -Other \& Unspecified Sites & $\begin{array}{l}190 \\
193-199\end{array}$ & 1 & 0 & 0 & 0 & 0 & 0 & 1 \\
\hline -Lymphatic \& Hematopoietic & $200-208$ & 0 & 0 & 0 & 0 & 1 & 0 & 1 \\
\hline BENIGN \& UNCERTAIN NEOPLASMS & $\begin{array}{l}\text { 210-229, } \\
235-239\end{array}$ & 1 & 0 & 0 & 0 & 1 & 1 & 3 \\
\hline ENDOCRINE/METABOLIC/IMMUNITY & $240-279$ & 8 & 0 & 2 & 2 & 1 & 6 & 19 \\
\hline -Thyroid Gland Disorders & $240-246$ & 1 & 0 & 0 & 0 & 0 & 1 & 2 \\
\hline -Other Endocrine Gland Dis & $250-259$ & 4 & 0 & 2 & 0 & 0 & 2 & 8 \\
\hline -Other Metabolic \& Immunity Disorders & $270-279$ & 3 & 0 & 0 & 2 & 1 & 3 & 9 \\
\hline BLOOD \& BLOOD-FORMING ORGANS & $280-289$ & 1 & 0 & 0 & 0 & 1 & 0 & 2 \\
\hline MENTAL DISORDERS & $290-319$ & 6 & 0 & 2 & 0 & 5 & 3 & 16 \\
\hline -Psychoses & $290-299$ & 0 & 0 & 0 & 0 & 1 & 0 & 1 \\
\hline -Non-Psychotic Disorders & $\begin{array}{l}300-302 \\
306-316\end{array}$ & 5 & 0 & 2 & 0 & 4 & 1 & 12 \\
\hline -Alcohol Dependence & 303 & 1 & 0 & 0 & 0 & 0 & 1 & 2 \\
\hline -Drug Dependence & $304-305$ & 0 & 0 & 0 & 0 & 0 & 1 & 1 \\
\hline $\begin{array}{l}\text { NERVOUS SYSTEM (NS) \& SENSE } \\
\text { ORGANS }\end{array}$ & $320-389$ & 10 & 3 & 0 & 0 & 6 & 18 & 37 \\
\hline -Other Disorders of Central NS & $340-349$ & 3 & 0 & 0 & 0 & 0 & 1 & 4 \\
\hline -Disorders of Peripheral NS & $350-359$ & 3 & 1 & 0 & 0 & 0 & 6 & 10 \\
\hline -Disorders of Eye & $360-379$ & 2 & 2 & 0 & 0 & 5 & 7 & 16 \\
\hline -Dis of Ear \& Mastoid & $380-389$ & 2 & 0 & 0 & 0 & 1 & 4 & 7 \\
\hline CIRCULATORY SYSTEM & $390-459$ & 22 & 1 & 7 & 3 & 3 & 11 & 47 \\
\hline -Chronic Rheumatic Heart Dis & 393-398 & 1 & 0 & 0 & 0 & 0 & 0 & 1 \\
\hline
\end{tabular}

(Continued)

*Only those diagnostic categories and gender/job category combinations with at least one occurrence appear in this table. 


\section{Nevada Test Site 2006}

\section{Absence Data}

Appendix I. Number of Diagnoses in Each Diagnostic Category by Gender and Job Category*

\begin{tabular}{|c|c|c|c|c|c|c|c|c|}
\hline & & \multicolumn{7}{|c|}{ Men } \\
\hline & & \multicolumn{6}{|c|}{ Job Category } & \multirow[b]{2}{*}{ TOTAL } \\
\hline & & Professional & $\begin{array}{l}\text { Administrative } \\
\text { Support }\end{array}$ & $\begin{array}{l}\text { Technical } \\
\text { Support }\end{array}$ & Service & $\begin{array}{c}\text { Security } \\
\text { and } \\
\text { Fire }\end{array}$ & Crafts & \\
\hline Diagnostic Category & \begin{tabular}{|l|} 
ICD-9-CM \\
Code
\end{tabular} & & & & & & & \\
\hline -Hypertensive Dis & $401-405$ & 7 & 1 & 1 & 1 & 1 & 4 & 15 \\
\hline -Ischemic Heart Dis & $410-414$ & 3 & 0 & 4 & 0 & 2 & 3 & 12 \\
\hline -Other Heart Dis & $420-429$ & 4 & 0 & 2 & 0 & 0 & 0 & 6 \\
\hline -Cerebrovascular Dis & $430-438$ & 0 & 0 & 0 & 0 & 0 & 2 & 2 \\
\hline -Dis of Arteries \& Capillaries & $440-448$ & 3 & 0 & 0 & 0 & 0 & 2 & 5 \\
\hline -Dis of Veins, Lymphatics, Other & $451-459$ & 4 & 0 & 0 & 2 & 0 & 0 & 6 \\
\hline RESPIRATORY SYSTEM & $460-519$ & 14 & 4 & 4 & 0 & 21 & 21 & 64 \\
\hline -Acute Respiratory Infections & $460-466$ & 5 & 1 & 1 & 0 & 5 & 9 & 21 \\
\hline -Other Dis Upper Respiratory Tract & $470-478$ & 3 & 1 & 1 & 0 & 7 & 4 & 16 \\
\hline -Pneumonia \& Influenza & $480-487$ & 4 & 0 & 1 & 0 & 1 & 6 & 12 \\
\hline -Chronic Obstructive Dis & $490-496$ & 1 & 1 & 0 & 0 & 4 & 2 & 8 \\
\hline -Lung Dis from External Agents & $500-508$ & 0 & 0 & 0 & 0 & 2 & 0 & 2 \\
\hline -Other Respiratory Dis & $510-519$ & 1 & 1 & 1 & 0 & 2 & 0 & 5 \\
\hline DIGESTIVE SYSTEM & $520-579$ & 19 & 2 & 12 & 1 & 9 & 8 & 51 \\
\hline -Oral Cavity, Saliva Glands, Jaw & $520-529$ & 0 & 0 & 1 & 0 & 0 & 0 & 1 \\
\hline -Esophagus, Stomach, Duodenum & $530-537$ & 1 & 1 & 2 & 0 & 1 & 2 & 7 \\
\hline -Appendicitis & $540-543$ & 0 & 0 & 2 & 0 & 0 & 0 & 2 \\
\hline -Hernia & $550-553$ & 7 & 1 & 5 & 0 & 2 & 2 & 17 \\
\hline -Enteritis, Colitis & $555-558$ & 2 & 0 & 1 & 0 & 5 & 1 & 9 \\
\hline -Other Intestinal Dis & $560-569$ & 2 & 0 & 0 & 0 & 0 & 1 & 3 \\
\hline -Other Digestive Dis & $570-579$ & 7 & 0 & 1 & 1 & 1 & 2 & 12 \\
\hline GENITOURINARY SYSTEM & $580-629$ & 6 & 0 & 2 & 1 & 3 & 2 & 14 \\
\hline -Nephritis, Nephrosis & $580-589$ & 0 & 0 & 1 & 0 & 0 & 0 & 1 \\
\hline -Other Urinary Dis & $590-599$ & 6 & 0 & 1 & 1 & 3 & 1 & 12 \\
\hline -Male Genital Organ Dis & $600-608$ & 0 & 0 & 0 & 0 & 0 & 1 & 1 \\
\hline SKIN \& SUBCUTANEOUS TISSUE & $680-709$ & 2 & 0 & 0 & 2 & 0 & 6 & 10 \\
\hline -Infections & $680-686$ & 1 & 0 & 0 & 2 & 0 & 4 & 7 \\
\hline -Other Inflammatory Conditions & $690-698$ & 1 & 0 & 0 & 0 & 0 & 1 & 2 \\
\hline -Other & 700-709 & 0 & 0 & 0 & 0 & 0 & 1 & 1 \\
\hline $\begin{array}{l}\text { MUSCULOSKELETAL \& CONNECTIVE } \\
\text { TISSUE }\end{array}$ & 710-739 & 25 & 1 & 7 & 2 & 24 & 26 & 85 \\
\hline -Arthropathies & $710-719$ & 6 & 0 & 3 & 0 & 13 & 7 & 29 \\
\hline -Dorsopathies & $720-724$ & 11 & 1 & 3 & 0 & 8 & 12 & 35 \\
\hline -Rheumatism, Excluding Back & 725-729 & 5 & 0 & 1 & 2 & 2 & 7 & 17 \\
\hline -Other Dis \& Acquired Deformities & $730-739$ & 3 & 0 & 0 & 0 & 1 & 0 & 4 \\
\hline
\end{tabular}

(Continued)

*Only those diagnostic categories and gender/job category combinations with at least one occurrence appear in this table. 


\section{Nevada Test Site 2006}

\section{Absence Data}

Appendix I. Number of Diagnoses in Each Diagnostic Category by Gender and Job Category*

\begin{tabular}{|c|c|c|c|c|c|c|c|c|}
\hline & & \multicolumn{7}{|c|}{ Men } \\
\hline & & \multicolumn{6}{|c|}{ Job Category } & \multirow[b]{2}{*}{ TOTAL } \\
\hline & & Professional & $\begin{array}{l}\text { Administrative } \\
\text { Support }\end{array}$ & $\begin{array}{l}\text { Technical } \\
\text { Support }\end{array}$ & Service & $\begin{array}{c}\text { Security } \\
\text { and } \\
\text { Fire }\end{array}$ & Crafts & \\
\hline Diagnostic Category & $\begin{array}{l}\text { ICD-9-CM } \\
\text { Code }\end{array}$ & & & & & & & \\
\hline CONGENITAL ANOMALIES & $740-759$ & 1 & 0 & 0 & 0 & 0 & 0 & 1 \\
\hline $\begin{array}{l}\text { SYMPTOMS, SIGNS, \& ILL-DEFINED } \\
\text { CONDITIONS }\end{array}$ & 780-799 & 13 & 5 & 15 & 3 & 21 & 24 & 81 \\
\hline -Symptoms & 780-789 & 12 & 5 & 15 & 3 & 20 & 23 & 78 \\
\hline -Non-Specific Abnormal Findings & $790-796$ & 1 & 0 & 0 & 0 & 1 & 0 & 2 \\
\hline -Ill-Defined \& Unknown Causes & 797-799 & 0 & 0 & 0 & 0 & 0 & 1 & 1 \\
\hline INJURY \& POISONING & $800-999$ & 18 & 0 & 6 & 3 & 27 & 33 & 87 \\
\hline -Fracture - Neck, Trunk & $805-809$ & 0 & 0 & 0 & 0 & 1 & 3 & 4 \\
\hline -Fracture - Upper Limb & $810-819$ & 1 & 0 & 0 & 0 & 0 & 1 & 2 \\
\hline -Fracture - Lower Limb & $820-829$ & 3 & 0 & 0 & 0 & 1 & 1 & 5 \\
\hline -Dislocation & $830-839$ & 3 & 0 & 1 & 0 & 1 & 6 & 11 \\
\hline -Sprains \& Strains - Back & 846-847 & 2 & 0 & 0 & 0 & 8 & 4 & 14 \\
\hline -Sprains \& Strains - Other & $\begin{array}{l}840-845 \\
848\end{array}$ & 8 & 0 & 3 & 0 & 8 & 6 & 25 \\
\hline -Intracranial Injury & $850-854$ & 0 & 0 & 0 & 1 & 0 & 0 & 1 \\
\hline -Internal Injury - Thorax, Abdomen, Pelvis & $860-869$ & 0 & 0 & 0 & 0 & 1 & 0 & 1 \\
\hline -Open Wound - Head, Neck, Trunk & $870-879$ & 0 & 0 & 0 & 1 & 1 & 1 & 3 \\
\hline -Open Wound - Upper Limb & $880-887$ & 0 & 0 & 0 & 0 & 0 & 1 & 1 \\
\hline -Open Wound - Lower Limb & $890-897$ & 0 & 0 & 0 & 0 & 1 & 0 & 1 \\
\hline -Superficial Injury & 910-919 & 0 & 0 & 0 & 0 & 1 & 1 & 2 \\
\hline -Contusion & $920-924$ & 0 & 0 & 1 & 1 & 1 & 2 & 5 \\
\hline -Burns & 940-949 & 0 & 0 & 0 & 0 & 0 & 1 & 1 \\
\hline -Injury to Nerves \& Spinal Cord & $950-957$ & 0 & 0 & 0 & 0 & 0 & 1 & 1 \\
\hline -Complications \& Unspecified Injuries & 958-959 & 0 & 0 & 0 & 0 & 2 & 4 & 6 \\
\hline -Toxic Effects - Non-medicinal & $980-989$ & 0 & 0 & 0 & 0 & 0 & 1 & 1 \\
\hline -Unspecified Effects - External Causes & $990-995$ & 0 & 0 & 1 & 0 & 0 & 0 & 1 \\
\hline -Complications of Surgical/Medical Care & 996-999 & 1 & 0 & 0 & 0 & 1 & 0 & 2 \\
\hline $\begin{array}{l}\text { HEALTH STATUS/HEALTH SERVICE } \\
\text { CONTACT }\end{array}$ & V01-V82 & 4 & 0 & 0 & 0 & 3 & 3 & 10 \\
\hline -Personal \& Family History & V10-V19 & 3 & 0 & 0 & 0 & 1 & 1 & 5 \\
\hline -Health Services Reproduction/Development & V20-V29 & 0 & 0 & 0 & 0 & 2 & 0 & 2 \\
\hline -Other Circumstances & V60-V69 & 1 & 0 & 0 & 0 & 0 & 1 & 2 \\
\hline -Examination \& Investigation & V70-V82 & 0 & 0 & 0 & 0 & 0 & 1 & 1 \\
\hline
\end{tabular}

*Only those diagnostic categories and gender/job category combinations with at least one occurrence appear in this table. 


\section{Nevada Test Site 2006}

Absence Data

Appendix I. Number of Diagnoses in Each Diagnostic Category by Gender and Job Category*

\begin{tabular}{|c|c|c|c|c|c|c|c|}
\hline & \multicolumn{7}{|c|}{ Men } \\
\hline & \multicolumn{6}{|c|}{ Job Category } & \multirow[b]{2}{*}{ TOTAL } \\
\hline & Professional & $\begin{array}{c}\text { Administrative } \\
\text { Support }\end{array}$ & $\begin{array}{l}\text { Technical } \\
\text { Support }\end{array}$ & Service & $\begin{array}{c}\text { Security } \\
\text { and } \\
\text { Fire }\end{array}$ & Crafts & \\
\hline $\begin{array}{l}\text { Diagnostic } \\
\text { Category }\end{array}$ & \multirow[b]{2}{*}{161} & \multirow[b]{2}{*}{16} & \multirow[b]{2}{*}{59} & \multirow[b]{2}{*}{17} & \multirow[b]{2}{*}{130} & \multirow[b]{2}{*}{168} & \multirow[b]{2}{*}{551} \\
\hline Total & & & & & & & \\
\hline
\end{tabular}

*Only those diagnostic categories and gender/job category combinations with at least one occurrence appear in this table. 


\section{Nevada Test Site 2006}

\section{Absence Data}

Appendix J. Total Number of Calendar Days Absent in Each Diagnostic Category by Gender and Job Category*

\begin{tabular}{|c|c|c|c|c|c|c|c|c|}
\hline & & \multicolumn{7}{|c|}{ Women } \\
\hline & & \multicolumn{6}{|c|}{ Job Category } & \multirow[b]{2}{*}{ TOTAL } \\
\hline & & Professional & $\begin{array}{c}\text { Administrative } \\
\text { Support }\end{array}$ & $\begin{array}{l}\text { Technical } \\
\text { Support }\end{array}$ & Service & \begin{tabular}{|c|}
$\begin{array}{c}\text { Security } \\
\text { and } \\
\text { Fire }\end{array}$ \\
\end{tabular} & Crafts & \\
\hline Diagnostic Category & $\begin{array}{l}\text { ICD-9-CM } \\
\text { Code }\end{array}$ & & & & & & & \\
\hline $\begin{array}{l}\text { INFECTIOUS \& PARASITIC DISEASES } \\
\text { (DIS) }\end{array}$ & 001-139 & 17 & 112 & 3 & 0 & 0 & 13 & 145 \\
\hline MALIGNANT NEOPLASMS & \begin{tabular}{|l}
$140-208$ \\
$230-234$
\end{tabular} & 163 & 0 & 358 & 0 & 0 & 0 & 521 \\
\hline BENIGN \& UNCERTAIN NEOPLASMS & $\begin{array}{l}210-229 \\
235-239\end{array}$ & 162 & 190 & 0 & 0 & 0 & 14 & 366 \\
\hline ENDOCRINE/METABOLIC/IMMUNITY & $240-279$ & 78 & 84 & 0 & 0 & 0 & 0 & 162 \\
\hline BLOOD \& BLOOD-FORMING ORGANS & $280-289$ & 19 & 0 & 0 & 0 & 0 & 0 & 19 \\
\hline MENTAL DISORDERS & $290-319$ & 222 & 273 & 93 & 0 & 0 & 80 & 668 \\
\hline $\begin{array}{l}\text { NERVOUS SYSTEM (NS) \& SENSE } \\
\text { ORGANS }\end{array}$ & 320-389 & 232 & 196 & 0 & 0 & 5 & 4 & 437 \\
\hline CIRCULATORY SYSTEM & $390-459$ & 109 & 134 & 1 & 0 & 42 & 61 & 347 \\
\hline RESPIRATORY SYSTEM & $460-519$ & 76 & 91 & 39 & 15 & 4 & 31 & 256 \\
\hline DIGESTIVE SYSTEM & $520-579$ & 144 & 110 & 55 & 0 & 0 & 68 & 377 \\
\hline GENITOURINARY SYSTEM & 580-629 & 314 & 379 & 47 & 0 & 0 & 56 & 796 \\
\hline SKIN \& SUBCUTANEOUS TISSUE & 680-709 & 0 & 121 & 14 & 0 & 0 & 0 & 135 \\
\hline $\begin{array}{l}\text { MUSCULOSKELETAL \& CONNECTIVE } \\
\text { TISSUE }\end{array}$ & 710-739 & 337 & 512 & 128 & 0 & 0 & 107 & 1,084 \\
\hline $\begin{array}{l}\text { SYMPTOMS, SIGNS, \& ILL-DEFINED } \\
\text { CONDITIONS }\end{array}$ & 780-799 & 221 & 267 & 161 & 0 & 23 & 58 & 730 \\
\hline INJURY \& POISONING & $800-999$ & 216 & 155 & 137 & 0 & 0 & 119 & 627 \\
\hline
\end{tabular}

\begin{tabular}{|c|c|c|c|c|c|c|c|c|}
\hline & & \multicolumn{7}{|c|}{ Men } \\
\hline & & \multicolumn{6}{|c|}{ Job Category } & \multirow[b]{2}{*}{ TOTAL } \\
\hline & & Professional & $\begin{array}{c}\text { Administrative } \\
\text { Support }\end{array}$ & $\begin{array}{l}\text { Technical } \\
\text { Support }\end{array}$ & Service & \begin{tabular}{|c|} 
Security \\
and \\
Fire
\end{tabular} & Crafts & \\
\hline Diagnostic Category & $\begin{array}{l}\text { ICD-9-CM } \\
\text { Code }\end{array}$ & & & & & & & \\
\hline $\begin{array}{l}\text { INFECTIOUS \& PARASITIC DISEASES } \\
\text { (DIS) }\end{array}$ & 001-139 & 59 & 0 & 11 & 0 & 14 & 41 & 125 \\
\hline MALIGNANT NEOPLASMS & \begin{tabular}{|l}
$140-208$ \\
$230-234$
\end{tabular} & 271 & 0 & 0 & 0 & 33 & 1 & 305 \\
\hline BENIGN \& UNCERTAIN NEOPLASMS & \begin{tabular}{|l|} 
210-229, \\
$235-239$ \\
\end{tabular} & 28 & 0 & 0 & 0 & 13 & 77 & 118 \\
\hline ENDOCRINE/METABOLIC/IMMUNITY & $240-279$ & 244 & 0 & 49 & 31 & 12 & 167 & 503 \\
\hline BLOOD \& BLOOD-FORMING ORGANS & $280-289$ & 28 & 0 & 0 & 0 & 7 & 0 & 35 \\
\hline MENTAL DISORDERS & $290-319$ & 331 & 0 & 21 & 0 & 48 & 10 & 410 \\
\hline
\end{tabular}

(Continued) 


\section{Nevada Test Site 2006}

Absence Data

Appendix J. Total Number of Calendar Days Absent in Each Diagnostic Category by Gender and Job Category*

\begin{tabular}{|c|c|c|c|c|c|c|c|c|}
\hline & & \multicolumn{7}{|c|}{ Men } \\
\hline & & \multicolumn{6}{|c|}{ Job Category } & \multirow[b]{2}{*}{ TOTAL } \\
\hline & & Professional & $\begin{array}{l}\text { Administrative } \\
\text { Support }\end{array}$ & $\begin{array}{l}\text { Technical } \\
\text { Support }\end{array}$ & Service & $\begin{array}{c}\text { Security } \\
\text { and } \\
\text { Fire }\end{array}$ & Crafts & \\
\hline Diagnostic Category & $\begin{array}{l}\text { ICD-9-CM } \\
\text { Code }\end{array}$ & & & & & & & \\
\hline $\begin{array}{l}\text { NERVOUS SYSTEM (NS) \& SENSE } \\
\text { ORGANS }\end{array}$ & $320-389$ & 72 & 82 & 0 & 0 & 45 & 145 & 344 \\
\hline CIRCULATORY SYSTEM & $390-459$ & 500 & 1 & 160 & 38 & 10 & 130 & 839 \\
\hline RESPIRATORY SYSTEM & $460-519$ & 80 & 16 & 46 & 0 & 124 & 203 & 469 \\
\hline DIGESTIVE SYSTEM & $520-579$ & 327 & 11 & 188 & 11 & 98 & 101 & 736 \\
\hline GENITOURINARY SYSTEM & $580-629$ & 82 & 0 & 43 & 8 & 10 & 13 & 156 \\
\hline SKIN \& SUBCUTANEOUS TISSUE & $680-709$ & 15 & 0 & 0 & 31 & 0 & 57 & 103 \\
\hline $\begin{array}{l}\text { MUSCULOSKELETAL \& CONNECTIVE } \\
\text { TISSUE }\end{array}$ & $710-739$ & 1,021 & 5 & 82 & 25 & 402 & 700 & 2,235 \\
\hline CONGENITAL ANOMALIES & $740-759$ & 12 & 0 & 0 & 0 & 0 & 0 & 12 \\
\hline $\begin{array}{l}\text { SYMPTOMS, SIGNS, \& ILL-DEFINED } \\
\text { CONDITIONS }\end{array}$ & 780-799 & 258 & 13 & 67 & 20 & 109 & 318 & 785 \\
\hline INJURY \& POISONING & 800-999 & 275 & 0 & 60 & 19 & 592 & 1,116 & 2,062 \\
\hline
\end{tabular}

\footnotetext{
*Absences with >1 ICD-9-CM code in the same diagnostic category were counted only once. Only those diagnostic categories and gender/job category combinations with at least one occurrence appear in this table.
} 


\section{Nevada Test Site 2006}

Absence Data

Appendix K. Age-Adjusted IIlness and Injury Rates by Diagnostic Category

Part 1. Men

\begin{tabular}{|c|c|c|c|c|c|}
\hline Category of Diagnoses & ICD-9-CM Code & $\begin{array}{c}\text { Number of } \\
\text { Diagnoses }\end{array}$ & $\begin{array}{l}\text { Age-Adjusted } \\
\text { Rate per 1000* }\end{array}$ & \begin{tabular}{|c|} 
Lower $95 \%$ \\
Confidence \\
Limit per 1000
\end{tabular} & $\begin{array}{c}\text { Upper } 95 \% \\
\text { Confidence } \\
\text { Limit per } 1000\end{array}$ \\
\hline Infections/Parasites & $001-139$ & 15 & 5.9 & 3.3 & 10.5 \\
\hline Cancer & $140-208,230-234$ & 9 & 1.9 & 1.0 & 3.7 \\
\hline -Lip, Oral Cavity, Pharynx & $140-149$ & 0 & 0 & 0 & 0 \\
\hline -Digestive Organs & $150-159$ & 2 & 0.4 & 0.1 & 1.5 \\
\hline -Respiratory System & $160-165$ & 0 & 0 & 0 & 0 \\
\hline -Bone, Connective Tissue, Skin & $170-173,176$ & 1 & 0.3 & 0.0 & 1.9 \\
\hline -Breast & $174-175$ & 0 & 0 & 0 & 0 \\
\hline -Genitourinary & $179-189$ & 4 & 0.8 & 0.3 & 2.2 \\
\hline -Other \& Unspecified Sites & $190,193-199$ & 1 & 0.2 & 0.0 & 1.3 \\
\hline -Nervous System & $191-192$ & 0 & 0 & 0 & 0 \\
\hline -Leukemia, Lymphoma & $200-208$ & 1 & 0.3 & 0.0 & 1.9 \\
\hline -Carcinoma in situ & $230-234$ & 0 & 0 & 0 & 0 \\
\hline Benign Growths & $210-229,235-239$ & 3 & 1.2 & 0.4 & 4.0 \\
\hline Endocrine/Metabolic & $240-279$ & 19 & 4.8 & 2.9 & 7.7 \\
\hline -Other Endocrine Glands & $250-259$ & 8 & 1.9 & 0.9 & 3.8 \\
\hline Blood & $280-289$ & 2 & 0.5 & 0.1 & 2.1 \\
\hline Mental & $290-319$ & 16 & 4.7 & 2.8 & 7.8 \\
\hline -Non-Psychotic Conditions & $300-302,306-316$ & 12 & 3.6 & 2.0 & 6.6 \\
\hline Nervous System & $320-389$ & 37 & 11.2 & 7.8 & 16.1 \\
\hline -Disorders of Peripheral NS & $350-359$ & 10 & 2.8 & 1.5 & 5.6 \\
\hline Heart/Circulatory & $390-459$ & 47 & 12.9 & 9.5 & 17.6 \\
\hline -Hypertensive Disease & $401-405$ & 15 & 4.3 & 2.5 & 7.4 \\
\hline -Ischemic Heart Disease & $410-414$ & 12 & 2.9 & 1.6 & 5.2 \\
\hline Respiratory & $460-519$ & 64 & 23.9 & 18.2 & 31.5 \\
\hline -Chronic Obstructive Diseases & $490-496$ & 8 & 3.4 & 1.6 & 7.5 \\
\hline -Lung Disease from External Agents & $500-508$ & 2 & 0.6 & 0.1 & 2.4 \\
\hline Digestive & $520-579$ & 51 & 15.8 & 11.6 & 21.5 \\
\hline -Hernias & $550-553$ & 17 & 6.2 & 3.7 & 10.6 \\
\hline Genitourinary & $580-629$ & 14 & 4.3 & 2.4 & 7.8 \\
\hline -Nephritis, Nephrosis & $580-589$ & 1 & 0.2 & 0.0 & 1.3 \\
\hline Miscarriage $^{* * *}$ & $630-677$ & 0 & 0 & 0 & 0 \\
\hline Skin & $680-709$ & 10 & 3.1 & 1.6 & 6.0 \\
\hline Musculoskeletal & $710-739$ & 85 & 31.7 & 24.9 & 40.4 \\
\hline -Arthropathies & $710-719$ & 29 & 10.1 & 6.6 & 15.4 \\
\hline -Dorsopathies & $720-724$ & 35 & 14.3 & 9.9 & 20.7 \\
\hline -Rheumatism, Excluding Back & $725-729$ & 17 & 6.0 & 3.6 & 10.1 \\
\hline Congenital Anomalies & $740-759$ & 1 & 0.3 & 0.0 & 2.1 \\
\hline
\end{tabular}

*Standardized to age distribution of 2000 U.S. population.

**Only women aged 18-45 were included in the calculation of the rates for these diagnostic categories. 


\section{Nevada Test Site 2006}

\section{Absence Data}

Appendix K. Age-Adjusted Illness and Injury Rates by Diagnostic Category

\begin{tabular}{|c|c|c|c|c|c|}
\hline Category of Diagnoses & ICD-9-CM Code & $\begin{array}{l}\text { Number of } \\
\text { Diagnoses }\end{array}$ & $\begin{array}{l}\text { Age-Adjusted } \\
\text { Rate per } 1000 *\end{array}$ & $\begin{array}{c}\text { Lower } 95 \% \\
\text { Confidence } \\
\text { Limit per } 1000\end{array}$ & $\begin{array}{c}\text { Upper } 95 \% \\
\text { Confidence } \\
\text { Limit per } 1000\end{array}$ \\
\hline Unspecified Symptoms & $780-799$ & 81 & 28.9 & 22.6 & 37.0 \\
\hline Injury & $800-999$ & 87 & 32.3 & 25.3 & 41.1 \\
\hline -Fractures - Skull, Neck, Trunk & $800-809$ & 4 & 1.4 & 0.4 & 4.4 \\
\hline -Fractures - Upper Limb & $810-819$ & 2 & 0.5 & 0.1 & 2.0 \\
\hline -Fractures - Lower Limb & $820-829$ & 5 & 1.5 & 0.6 & 4.1 \\
\hline -Dislocations & $830-839$ & 11 & 3.5 & 1.8 & 6.9 \\
\hline -Back Sprains \& Strains & $846-847$ & 14 & 6.6 & 3.7 & 11.7 \\
\hline -Other Sprains \& Strains & $840-845,848$ & 25 & 7.3 & 4.6 & 11.6 \\
\hline -Open Wounds & $870-887,890-897$ & 5 & 2.0 & 0.7 & 5.4 \\
\hline -Burns & $940-949$ & 1 & 0.3 & 0.0 & 1.9 \\
\hline -Adverse Reactions to Nonmedical Substances & $980-989$ & 1 & 0.3 & 0.0 & 2.1 \\
\hline -Adverse Reactions to External Causes & $990-995$ & 1 & 0.3 & 0.0 & 2.1 \\
\hline Health Status/Health Service Contact & V01-V82 & 0 & 0 & 0 & 0 \\
\hline Total & & 541 & 183.5 & 166.8 & 201.9 \\
\hline
\end{tabular}

Part 2. Women

\begin{tabular}{|c|c|c|c|c|c|}
\hline Category of Diagnoses & ICD-9-CM Code & $\begin{array}{l}\text { Number of } \\
\text { Diagnoses }\end{array}$ & $\begin{array}{l}\text { Age-Adjusted } \\
\text { Rate per } 1000 *\end{array}$ & \begin{tabular}{|c|} 
Lower $95 \%$ \\
Confidence \\
Limit per 1000
\end{tabular} & $\begin{array}{c}\text { Upper } 95 \% \\
\text { Confidence } \\
\text { Limit per } 1000\end{array}$ \\
\hline Infections/Parasites & 001-139 & 10 & 10.3 & 4.9 & 21.6 \\
\hline Cancer & $140-208,230-234$ & 4 & 2.1 & 0.8 & 5.7 \\
\hline -Lip, Oral Cavity, Pharynx & $140-149$ & 0 & 0 & 0 & 0 \\
\hline -Digestive Organs & $150-159$ & 0 & 0 & 0 & 0 \\
\hline -Respiratory System & $160-165$ & 0 & 0 & 0 & 0 \\
\hline -Bone, Connective Tissue, Skin & $170-173,176$ & 0 & 0 & 0 & 0 \\
\hline -Breast & $174-175$ & 2 & 1.1 & 0.3 & 4.6 \\
\hline -Genitourinary & $179-189$ & 0 & 0 & 0 & 0 \\
\hline -Other \& Unspecified Sites & $190,193-199$ & 1 & 0.5 & 0.1 & 3.5 \\
\hline -Nervous System & 191-192 & 0 & 0 & 0 & 0 \\
\hline -Leukemia, Lymphoma & $200-208$ & 1 & 0.5 & 0.1 & 3.5 \\
\hline -Carcinoma in situ & $230-234$ & 0 & 0 & 0 & 0 \\
\hline Benign Growths & $210-229,235-239$ & 10 & 7.5 & 3.9 & 14.6 \\
\hline Endocrine/Metabolic & $240-279$ & 13 & 10.8 & 6.1 & 19.2 \\
\hline -Other Endocrine Glands & $250-259$ & 5 & 3.3 & 1.4 & 8.2 \\
\hline Blood & $280-289$ & 2 & 2.7 & 0.5 & 14.2 \\
\hline Mental & $290-319$ & 18 & 12.2 & 7.4 & 20.1 \\
\hline -Non-Psychotic Conditions & $300-302,306-316$ & 15 & 10.4 & 6.0 & 18.0 \\
\hline Nervous System & $320-389$ & 18 & 15.6 & 9.6 & 25.3 \\
\hline
\end{tabular}

*Standardized to age distribution of 2000 U.S. population.

**Only women aged 18-45 were included in the calculation of the rates for these diagnostic categories. 


\section{Nevada Test Site 2006}

Absence Data

Appendix K. Age-Adjusted IIlness and Injury Rates by Diagnostic Category

\begin{tabular}{|c|c|c|c|c|c|}
\hline Category of Diagnoses & ICD-9-CM Code & $\begin{array}{l}\text { Number of } \\
\text { Diagnoses }\end{array}$ & $\begin{array}{l}\text { Age-Adjusted } \\
\text { Rate per } 1000 *\end{array}$ & \begin{tabular}{|c|} 
Lower $95 \%$ \\
Confidence \\
Limit per 1000
\end{tabular} & $\begin{array}{c}\text { Upper } 95 \% \\
\text { Confidence } \\
\text { Limit per } 1000\end{array}$ \\
\hline -Disorders of Peripheral NS & $350-359$ & 8 & 8.0 & 3.9 & 16.4 \\
\hline Heart/Circulatory & $390-459$ & 17 & 11.2 & 6.8 & 18.2 \\
\hline -Hypertensive Disease & $401-405$ & 13 & 8.5 & 4.8 & 14.8 \\
\hline -Ischemic Heart Disease & $410-414$ & 2 & 1.6 & 0.4 & 6.4 \\
\hline Respiratory & $460-519$ & 33 & 33.7 & 22.9 & 49.6 \\
\hline -Chronic Obstructive Diseases & $490-496$ & 6 & 5.4 & 2.4 & 12.4 \\
\hline -Lung Disease from External Agents & $500-508$ & 0 & 0 & 0 & 0 \\
\hline Digestive & $520-579$ & 24 & 19.4 & 12.4 & 30.3 \\
\hline -Hernias & $550-553$ & 1 & 0.5 & 0.1 & 3.5 \\
\hline Genitourinary & $580-629$ & 31 & 34.9 & 23.5 & 52.0 \\
\hline -Nephritis, Nephrosis & $580-589$ & 0 & 0 & 0 & 0 \\
\hline Miscarriage*** & $630-677$ & 0 & 0 & 0 & 0 \\
\hline Skin & $680-709$ & 5 & 4.3 & 1.7 & 10.7 \\
\hline Musculoskeletal & $710-739$ & 62 & 54.8 & 41.8 & 71.9 \\
\hline -Arthropathies & $710-719$ & 19 & 12.9 & 8.0 & 20.8 \\
\hline -Dorsopathies & $720-724$ & 29 & 30.7 & 20.8 & 45.4 \\
\hline -Rheumatism, Excluding Back & $725-729$ & 11 & 8.4 & 4.4 & 15.9 \\
\hline Congenital Anomalies & $740-759$ & 0 & 0 & 0 & 0 \\
\hline Unspecified Symptoms & $780-799$ & 57 & 44.1 & 33.2 & 58.5 \\
\hline Injury & $800-999$ & 31 & 25.7 & 17.4 & 37.8 \\
\hline -Fractures - Skull, Neck, Trunk & $800-809$ & 1 & 0.9 & 0.1 & 6.5 \\
\hline -Fractures - Upper Limb & $810-819$ & 3 & 2.3 & 0.6 & 8.0 \\
\hline -Fractures - Lower Limb & $820-829$ & 5 & 2.9 & 1.2 & 7.1 \\
\hline -Dislocations & $830-839$ & 3 & 2.2 & 0.7 & 6.9 \\
\hline -Back Sprains \& Strains & $846-847$ & 2 & 1.8 & 0.4 & 8.1 \\
\hline -Other Sprains \& Strains & $840-845,848$ & 7 & 5.4 & 2.5 & 11.8 \\
\hline -Open Wounds & $870-887,890-897$ & 0 & 0 & 0 & 0 \\
\hline -Burns & $940-949$ & 0 & 0 & 0 & 0 \\
\hline -Adverse Reactions to Nonmedical Substances & $980-989$ & 0 & 0 & 0 & 0 \\
\hline -Adverse Reactions to External Causes & $990-995$ & 1 & 0.9 & 0.1 & 6.5 \\
\hline Health Status/Health Service Contact & V01-V82 & 0 & 0 & 0 & 0 \\
\hline Total & & 335 & 289.4 & 256.8 & 326.1 \\
\hline
\end{tabular}

*Standardized to age distribution of 2000 U.S. population.

**Only women aged 18-45 were included in the calculation of the rates for these diagnostic categories. 


\section{Nevada Test Site 2006}

\section{Absence Data}

Appendix K. Age-Adjusted IIIness and Injury Rates by Diagnostic Category

Part 3. Men and Women

\begin{tabular}{|c|c|c|c|c|c|}
\hline Category of Diagnoses & ICD-9-CM Code & $\begin{array}{c}\text { Number of } \\
\text { Diagnoses }\end{array}$ & $\begin{array}{l}\text { Age-Adjusted } \\
\text { Rate per 1000* }\end{array}$ & \begin{tabular}{|c|} 
Lower $95 \%$ \\
Confidence \\
Limit per 1000
\end{tabular} & $\begin{array}{c}\text { Upper } 95 \% \\
\text { Confidence } \\
\text { Limit per } 1000\end{array}$ \\
\hline Infections/Parasites & $001-139$ & 25 & 7.1 & 4.5 & 11.2 \\
\hline Cancer & $140-208,230-234$ & 13 & 2.1 & 1.2 & 3.6 \\
\hline -Lip, Oral Cavity, Pharynx & $140-149$ & 0 & 0 & 0 & 0 \\
\hline -Digestive Organs & $150-159$ & 2 & 0.3 & 0.1 & 1.1 \\
\hline -Respiratory System & $160-165$ & 0 & 0 & 0 & 0 \\
\hline -Bone, Connective Tissue, Skin & $170-173,176$ & 1 & 0.2 & 0.0 & 1.5 \\
\hline -Breast & $174-175$ & 2 & 0.3 & 0.1 & 1.4 \\
\hline -Genitourinary & $179-189$ & 4 & 0.6 & 0.2 & 1.7 \\
\hline -Other \& Unspecified Sites & $190,193-199$ & 2 & 0.3 & 0.1 & 1.1 \\
\hline -Nervous System & $191-192$ & 0 & 0 & 0 & 0 \\
\hline -Leukemia, Lymphoma & $200-208$ & 2 & 0.3 & 0.1 & 1.4 \\
\hline -Carcinoma in situ & $230-234$ & 0 & 0 & 0 & 0 \\
\hline Benign Growths & $210-229,235-239$ & 13 & 3.2 & 1.8 & 5.7 \\
\hline Endocrine/Metabolic & $240-279$ & 32 & 6.7 & 4.6 & 9.7 \\
\hline -Other Endocrine Glands & $250-259$ & 13 & 2.4 & 1.4 & 4.2 \\
\hline Blood & $280-289$ & 4 & 1.1 & 0.4 & 3.4 \\
\hline Mental & $290-319$ & 34 & 6.9 & 4.8 & 9.9 \\
\hline -Non-Psychotic Conditions & $300-302,306-316$ & 27 & 5.6 & 3.7 & 8.5 \\
\hline Nervous System & $320-389$ & 55 & 12.4 & 9.3 & 16.6 \\
\hline -Disorders of Peripheral NS & $350-359$ & 18 & 4.3 & 2.6 & 7.1 \\
\hline Heart/Circulatory & $390-459$ & 64 & 12.6 & 9.7 & 16.3 \\
\hline -Hypertensive Disease & $401-405$ & 28 & 5.5 & 3.7 & 8.1 \\
\hline -Ischemic Heart Disease & $410-414$ & 14 & 2.6 & 1.6 & 4.5 \\
\hline Respiratory & $460-519$ & 97 & 26.6 & 21.3 & 33.3 \\
\hline -Chronic Obstructive Diseases & $490-496$ & 14 & 4.0 & 2.2 & 7.1 \\
\hline -Lung Disease from External Agents & $500-508$ & 2 & 0.4 & 0.1 & 1.6 \\
\hline Digestive & $520-579$ & 75 & 16.7 & 13.0 & 21.5 \\
\hline -Hernias & $550-553$ & 18 & 4.5 & 2.7 & 7.6 \\
\hline Genitourinary & $580-629$ & 45 & 13.1 & 9.4 & 18.2 \\
\hline -Nephritis, Nephrosis & $580-589$ & 1 & 0.1 & 0.0 & 1.0 \\
\hline Miscarriage $^{* * *}$ & $630-677$ & 0 & 0 & 0 & 0 \\
\hline Skin & $680-709$ & 15 & 3.3 & 2.0 & 5.7 \\
\hline Musculoskeletal & $710-739$ & 147 & 39.4 & 32.9 & 47.3 \\
\hline -Arthropathies & $710-719$ & 48 & 11.0 & 7.9 & 15.3 \\
\hline -Dorsopathies & $720-724$ & 64 & 19.7 & 15.1 & 25.8 \\
\hline -Rheumatism, Excluding Back & $725-729$ & 28 & 6.9 & 4.6 & 10.4 \\
\hline Congenital Anomalies & $740-759$ & 1 & 0.2 & 0.0 & 1.4 \\
\hline
\end{tabular}

*Standardized to age distribution of 2000 U.S. population.

**Only women aged 18-45 were included in the calculation of the rates for these diagnostic categories. 
Nevada Test Site 2006

Absence Data

Appendix K. Age-Adjusted Illness and Injury Rates by Diagnostic Category

\begin{tabular}{|c|c|c|c|c|c|}
\hline Category of Diagnoses & ICD-9-CM Code & $\begin{array}{l}\text { Number of } \\
\text { Diagnoses }\end{array}$ & $\begin{array}{c}\text { Age-Adjusted } \\
\text { Rate per } 1000 *\end{array}$ & \begin{tabular}{c|} 
Lower $95 \%$ \\
Confidence \\
Limit per 1000
\end{tabular} & $\begin{array}{c}\text { Upper } 95 \% \\
\text { Confidence } \\
\text { Limit per } 1000\end{array}$ \\
\hline Unspecified Symptoms & $780-799$ & 138 & 32.9 & 27.3 & 39.8 \\
\hline Injury & $800-999$ & 118 & 30.8 & 25.1 & 37.9 \\
\hline -Fractures - Skull, Neck, Trunk & $800-809$ & 5 & 1.2 & 0.4 & 3.4 \\
\hline -Fractures - Upper Limb & $810-819$ & 5 & 1.0 & 0.4 & 2.7 \\
\hline -Fractures - Lower Limb & $820-829$ & 10 & 2.0 & 1.0 & 3.8 \\
\hline -Dislocations & $830-839$ & 14 & 3.2 & 1.7 & 5.8 \\
\hline -Back Sprains \& Strains & $846-847$ & 16 & 5.3 & 3.1 & 9.2 \\
\hline -Other Sprains \& Strains & $840-845,848$ & 32 & 6.9 & 4.6 & 10.2 \\
\hline -Open Wounds & $870-887,890-897$ & 5 & 1.4 & 0.5 & 3.9 \\
\hline -Burns & $940-949$ & 1 & 0.2 & 0.0 & 1.5 \\
\hline -Adverse Reactions to Nonmedical Substances & $980-989$ & 1 & 0.2 & 0.0 & 1.4 \\
\hline -Adverse Reactions to External Causes & $990-995$ & 2 & 0.4 & 0.1 & 1.7 \\
\hline Health Status/Health Service Contact & V01-V82 & 0 & 0 & 0 & 0 \\
\hline Total & & 876 & 215.2 & 199.8 & 231.9 \\
\hline
\end{tabular}

*Standardized to age distribution of 2000 U.S. population.

**Only women aged 18-45 were included in the calculation of the rates for these diagnostic categories. 
Nevada Test Site 2006

OSHA Data

Appendix L. Number of Workers with at Least One OSHA Event by Gender, Age, and Job Category*

\begin{tabular}{|c|c|c|c|c|c|c|c|c|c|c|}
\hline \multirow{3}{*}{ Job Category } & \multicolumn{4}{|c|}{ Women } & \multicolumn{5}{|c|}{ Men } & \multirow[b]{3}{*}{ TOTAL } \\
\hline & \multicolumn{3}{|c|}{ Age Group } & \multirow[b]{2}{*}{ TOTAL } & \multicolumn{4}{|c|}{ Age Group } & \multirow[b]{2}{*}{ TOTAL } & \\
\hline & $30-39$ & $40-49$ & $\mathbf{5 0 +}$ & & $16-29$ & $30-39$ & $40-49$ & $50+$ & & \\
\hline Professional & 1 & 1 & 0 & 2 & 0 & 1 & 3 & 3 & 7 & 9 \\
\hline Administrative Support & 0 & 0 & 0 & 0 & 0 & 0 & 0 & 2 & 2 & 2 \\
\hline Technical Support & 0 & 0 & 3 & 3 & 0 & 0 & 0 & 0 & 0 & 3 \\
\hline Security and Fire & 0 & 1 & 0 & 1 & 6 & 5 & 8 & 2 & 21 & 22 \\
\hline Crafts & 2 & 3 & 3 & 8 & 2 & 3 & 7 & 15 & 27 & 35 \\
\hline TOTAL & 3 & 5 & 6 & 14 & 8 & 9 & 18 & 22 & 57 & 71 \\
\hline
\end{tabular}

*Only those job categories and gender/age combinations with at least one OSHA event appear in this table.

Appendix M. Total Number of Workdays Lost or with Restricted Activity from OSHA Events by Gender and Age

\begin{tabular}{|c|c|c|c|c|c|c|c|c|c|}
\hline \multirow{2}{*}{$\begin{array}{c}\text { Age } \\
\text { Group }\end{array}$} & \multicolumn{3}{|c|}{ Women } & \multicolumn{3}{|c|}{ Men } & \multicolumn{3}{|c|}{ TOTAL } \\
\hline & $\begin{array}{c}\text { Number } \\
\text { of } \\
\text { Events }\end{array}$ & $\begin{array}{c}\text { Days } \\
\text { Restricted }\end{array}$ & $\begin{array}{l}\text { Days } \\
\text { Lost }\end{array}$ & $\begin{array}{c}\text { Number } \\
\text { of } \\
\text { Events }\end{array}$ & $\begin{array}{c}\text { Days } \\
\text { Restricted }\end{array}$ & $\begin{array}{l}\text { Days } \\
\text { Lost }\end{array}$ & $\begin{array}{c}\text { Number } \\
\text { of } \\
\text { Events }\end{array}$ & $\begin{array}{c}\text { Days } \\
\text { Restricted }\end{array}$ & $\begin{array}{l}\text { Days } \\
\text { Lost }\end{array}$ \\
\hline $16-29$ & 0 & 0 & 0 & 8 & 7 & 3 & 8 & 7 & 3 \\
\hline $30-39$ & 3 & 0 & 0 & 9 & 260 & 98 & 12 & 260 & 98 \\
\hline $40-49$ & 5 & 14 & 136 & 19 & 351 & 226 & 24 & 365 & 362 \\
\hline $50+$ & 6 & 0 & 0 & 22 & 427 & 226 & 28 & 427 & 226 \\
\hline TOTAL & 14 & 14 & 136 & 58 & 1045 & 553 & 72 & 1059 & 689 \\
\hline
\end{tabular}

Appendix N. Total Number of Workdays Lost or with Restricted Activity from OSHA Events by Gender and Job Category*

\begin{tabular}{|l|r|r|r|r|r|r|r|r|r|}
\hline \multirow{2}{*}{ Job Category } & \multicolumn{4}{|c|}{ Women } & \multicolumn{3}{|c|}{ Men } & \multicolumn{3}{c|}{ Total } \\
\cline { 2 - 11 } & $\begin{array}{c}\text { Number } \\
\text { of } \\
\text { Events }\end{array}$ & $\begin{array}{c}\text { Days } \\
\text { Restricted }\end{array}$ & $\begin{array}{c}\text { Days } \\
\text { Lost }\end{array}$ & $\begin{array}{c}\text { Number } \\
\text { of } \\
\text { Events }\end{array}$ & $\begin{array}{c}\text { Days } \\
\text { Restricted }\end{array}$ & $\begin{array}{c}\text { Number } \\
\text { Days } \\
\text { Lost } \\
\text { Events }\end{array}$ & $\begin{array}{c}\text { Days } \\
\text { Restricted }\end{array}$ & $\begin{array}{c}\text { Days } \\
\text { Lost }\end{array}$ \\
\hline Professional & 2 & 0 & 0 & 7 & 7 & 0 & 9 & 7 & 0 \\
\hline Administrative Support & 0 & 0 & 0 & 2 & 0 & 26 & 2 & 0 & 26 \\
\hline Technical Support & 3 & 0 & 0 & 0 & 0 & 0 & 3 & 0 & 0 \\
\hline Security and Fire & 1 & 7 & 12 & 22 & 601 & 103 & 23 & 608 & 115 \\
\hline Crafts & 8 & 7 & 124 & 27 & 437 & 424 & 35 & 444 & 548 \\
\hline Total & 14 & 14 & 136 & 58 & 1045 & 553 & 72 & 1059 & 689 \\
\hline
\end{tabular}

*Only those job categories with at least one OSHA event appear in this table. 


\section{Nevada Test Site 2006}

OSHA Data

Appendix O. Number of Diagnoses in Each Diagnostic Category by Gender and Age*

\begin{tabular}{|c|c|c|c|c|c|c|c|c|c|c|c|}
\hline & & \multicolumn{4}{|c|}{ Women } & \multicolumn{5}{|c|}{ Men } & \multirow[b]{3}{*}{ TOTAL } \\
\hline & & \multicolumn{3}{|c|}{ Age Group } & \multirow[b]{2}{*}{ TOTAL } & \multicolumn{4}{|c|}{ Age Group } & \multirow[b]{2}{*}{ TOTAL } & \\
\hline & & $30-39$ & $40-49$ & $\begin{array}{c}50 \\
+\end{array}$ & & $16-29$ & $30-39$ & $40-49$ & $\begin{array}{c}50 \\
+ \\
\end{array}$ & & \\
\hline Diagnostic Category & $\begin{array}{l}\text { ICD-9-CM } \\
\text { code }\end{array}$ & & & & & & & & & & \\
\hline ENDOCRINE/METABOLIC/IMMUNITY & $240-279$ & 0 & 0 & 0 & 0 & 1 & 0 & 0 & 0 & 1 & 1 \\
\hline -Other Metabolic \& Immunity Disorders & $270-279$ & 0 & 0 & 0 & 0 & 1 & 0 & 0 & 0 & 1 & 1 \\
\hline $\begin{array}{l}\text { NERVOUS SYSTEM (NS) \& SENSE } \\
\text { ORGANS }\end{array}$ & 320-389 & 0 & 0 & 0 & 0 & 1 & 3 & 1 & 0 & 5 & 5 \\
\hline -Disorders of Peripheral NS & $350-359$ & 0 & 0 & 0 & 0 & 1 & 0 & 1 & 0 & 2 & 2 \\
\hline -Disorders of Eye & $360-379$ & 0 & 0 & 0 & 0 & 0 & 3 & 0 & 0 & 3 & 3 \\
\hline RESPIRATORY SYSTEM & $460-519$ & 0 & 0 & 0 & 0 & 0 & 0 & 3 & 0 & 3 & 3 \\
\hline -Other Dis Upper Respiratory Tract & $470-478$ & 0 & 0 & 0 & 0 & 0 & 0 & 2 & 0 & 2 & 2 \\
\hline -Other Respiratory Dis & $510-519$ & 0 & 0 & 0 & 0 & 0 & 0 & 1 & 0 & 1 & 1 \\
\hline DIGESTIVE SYSTEM & $520-579$ & 0 & 0 & 0 & 0 & 0 & 0 & 1 & 0 & 1 & 1 \\
\hline -Esophagus, Stomach, Duodenum & $530-537$ & 0 & 0 & 0 & 0 & 0 & 0 & 1 & 0 & 1 & 1 \\
\hline GENITOURINARY SYSTEM & $580-629$ & 0 & 0 & 0 & 0 & 0 & 1 & 0 & 0 & 1 & 1 \\
\hline -Other Urinary Dis & $590-599$ & 0 & 0 & 0 & 0 & 0 & 1 & 0 & 0 & 1 & 1 \\
\hline $\begin{array}{l}\text { MUSCULOSKELETAL \& CONNECTIVE } \\
\text { TISSUE }\end{array}$ & 710-739 & 2 & 3 & 2 & 7 & 4 & 5 & 9 & 11 & 29 & 36 \\
\hline -Arthropathies & $710-719$ & 2 & 1 & 2 & 5 & 2 & 0 & 2 & 7 & 11 & 16 \\
\hline -Dorsopathies & $720-724$ & 0 & 2 & 0 & 2 & 2 & 2 & 5 & 2 & 11 & 13 \\
\hline -Rheumatism, Excluding Back & $725-729$ & 0 & 0 & 0 & 0 & 0 & 3 & 2 & 2 & 7 & 7 \\
\hline $\begin{array}{l}\text { SYMPTOMS, SIGNS, \& ILL-DEFINED } \\
\text { CONDITIONS }\end{array}$ & $780-799$ & 0 & 1 & 2 & 3 & 2 & 0 & 5 & 2 & 9 & 12 \\
\hline -Symptoms & 780-789 & 0 & 1 & 2 & 3 & 2 & 0 & 5 & 2 & 9 & 12 \\
\hline INJURY \& POISONING & $800-999$ & 2 & 5 & 8 & 15 & 5 & 6 & 17 & 21 & 49 & 64 \\
\hline -Fracture - Upper Limb & 810-819 & 0 & 0 & 1 & 1 & 0 & 0 & 0 & 1 & 1 & 2 \\
\hline -Fracture - Lower Limb & $820-829$ & 0 & 0 & 0 & 0 & 0 & 1 & 0 & 0 & 1 & 1 \\
\hline -Dislocation & 830-839 & 0 & 0 & 0 & 0 & 0 & 0 & 2 & 3 & 5 & 5 \\
\hline -Sprains \& Strains - Back & 846-847 & 0 & 0 & 1 & 1 & 0 & 1 & 2 & 0 & 3 & 4 \\
\hline -Sprains \& Strains - Other & $\begin{array}{l}840-845 \\
848\end{array}$ & 0 & 2 & 1 & 3 & 1 & 0 & 3 & 3 & 7 & 10 \\
\hline -Open Wound - Head, Neck, Trunk & $870-879$ & 0 & 0 & 2 & 2 & 1 & 0 & 0 & 1 & 2 & 4 \\
\hline -Open Wound - Upper Limb & 880-887 & 0 & 0 & 0 & 0 & 1 & 0 & 0 & 2 & 3 & 3 \\
\hline -Open Wound - Lower Limb & 890-897 & 0 & 0 & 0 & 0 & 0 & 1 & 0 & 1 & 2 & 2 \\
\hline -Superficial Injury & 910-919 & 0 & 0 & 0 & 0 & 0 & 0 & 3 & 0 & 3 & 3 \\
\hline -Contusion & $920-924$ & 0 & 2 & 2 & 4 & 1 & 0 & 0 & 0 & 1 & 5 \\
\hline -Foreign Body Entering Orifice & 930-939 & 0 & 1 & 0 & 1 & 0 & 1 & 1 & 0 & 2 & 3 \\
\hline -Burns & 940-949 & 1 & 0 & 0 & 1 & 0 & 0 & 0 & 0 & 0 & 1 \\
\hline -Complications \& Unspecified Injuries & 958-959 & 1 & 0 & 1 & 2 & 1 & 2 & 5 & 10 & 18 & 20 \\
\hline -Unspecified Effects - External Causes & $990-995$ & 0 & 0 & 0 & 0 & 0 & 0 & 1 & 0 & 1 & 1 \\
\hline
\end{tabular}

*Only those diagnostic categories and gender/age combinations with at least one OSHA event appear in this table. 


\section{Nevada Test Site 2006}

OSHA Data

Appendix O. Number of Diagnoses in Each Diagnostic Category by Gender and Age*

\begin{tabular}{|c|c|c|c|c|c|c|c|c|c|c|}
\hline & \multicolumn{4}{|c|}{ Women } & \multicolumn{5}{|c|}{ Men } & \multirow[b]{3}{*}{ TOTAL } \\
\hline & \multicolumn{3}{|c|}{ Age Group } & \multirow[b]{2}{*}{ TOTAL } & \multicolumn{4}{|c|}{ Age Group } & \multirow[b]{2}{*}{ TOTAL } & \\
\hline & $30-39$ & $40-49$ & $50+$ & & $16-29$ & $30-39$ & $40-49$ & $50+$ & & \\
\hline $\begin{array}{l}\text { Diagnostic } \\
\text { Category }\end{array}$ & & & & & & & & & & \\
\hline Total & 4 & 9 & 12 & 25 & 13 & 15 & 36 & 34 & 98 & 123 \\
\hline
\end{tabular}

*Only those diagnostic categories and gender/age combinations with at least one OSHA event appear in this table. 


\section{Nevada Test Site 2006}

OSHA Data

Appendix P. Number of Workdays Lost or with Restricted Activity in Each Diagnostic Category by Gender and Age*

\begin{tabular}{|c|c|c|c|c|c|c|c|}
\hline & & \multicolumn{6}{|c|}{ Women } \\
\hline & & \multicolumn{6}{|c|}{ Age Group } \\
\hline & & \multicolumn{2}{|c|}{$30-39$} & \multicolumn{2}{|c|}{$40-49$} & \multicolumn{2}{|l|}{$50+$} \\
\hline & & $\begin{array}{c}\text { Days } \\
\text { Restricted }\end{array}$ & \begin{tabular}{l|} 
Days \\
Lost
\end{tabular} & \begin{tabular}{|c|} 
Days \\
Restricted
\end{tabular} & \begin{tabular}{l|} 
Days \\
Lost
\end{tabular} & $\begin{array}{c}\text { Days } \\
\text { Restricted }\end{array}$ & $\begin{array}{l}\text { Days } \\
\text { Lost }\end{array}$ \\
\hline Diagnostic Category & $\begin{array}{l}\text { ICD-9-CM } \\
\text { Codes }\end{array}$ & & & & & & \\
\hline -Arthropathies & $710-719$ & 0 & 0 & 7 & 12 & 0 & 0 \\
\hline -Dorsopathies & $720-724$ & 0 & 0 & 0 & 0 & 0 & 0 \\
\hline -Symptoms & 780-789 & 0 & 0 & 0 & 0 & 0 & 0 \\
\hline -Fracture - Upper Limb & $810-819$ & 0 & 0 & 0 & 0 & 0 & 0 \\
\hline -Sprains \& Strains - Back & 846-847 & 0 & 0 & 0 & 0 & 0 & 0 \\
\hline -Sprains \& Strains - Other & $840-845,848$ & 0 & 0 & 14 & 136 & 0 & 0 \\
\hline -Open Wound - Head, Neck, Trunk & $870-879$ & 0 & 0 & 0 & 0 & 0 & 0 \\
\hline -Contusion & $920-924$ & 0 & 0 & 7 & 124 & 0 & 0 \\
\hline -Foreign Body Entering Orifice & $930-939$ & 0 & 0 & 0 & 0 & 0 & 0 \\
\hline -Burns & $940-949$ & 0 & 0 & 0 & 0 & 0 & 0 \\
\hline -Complications \& Unspecified Injuries & 958-959 & 0 & 0 & 0 & 0 & 0 & 0 \\
\hline
\end{tabular}

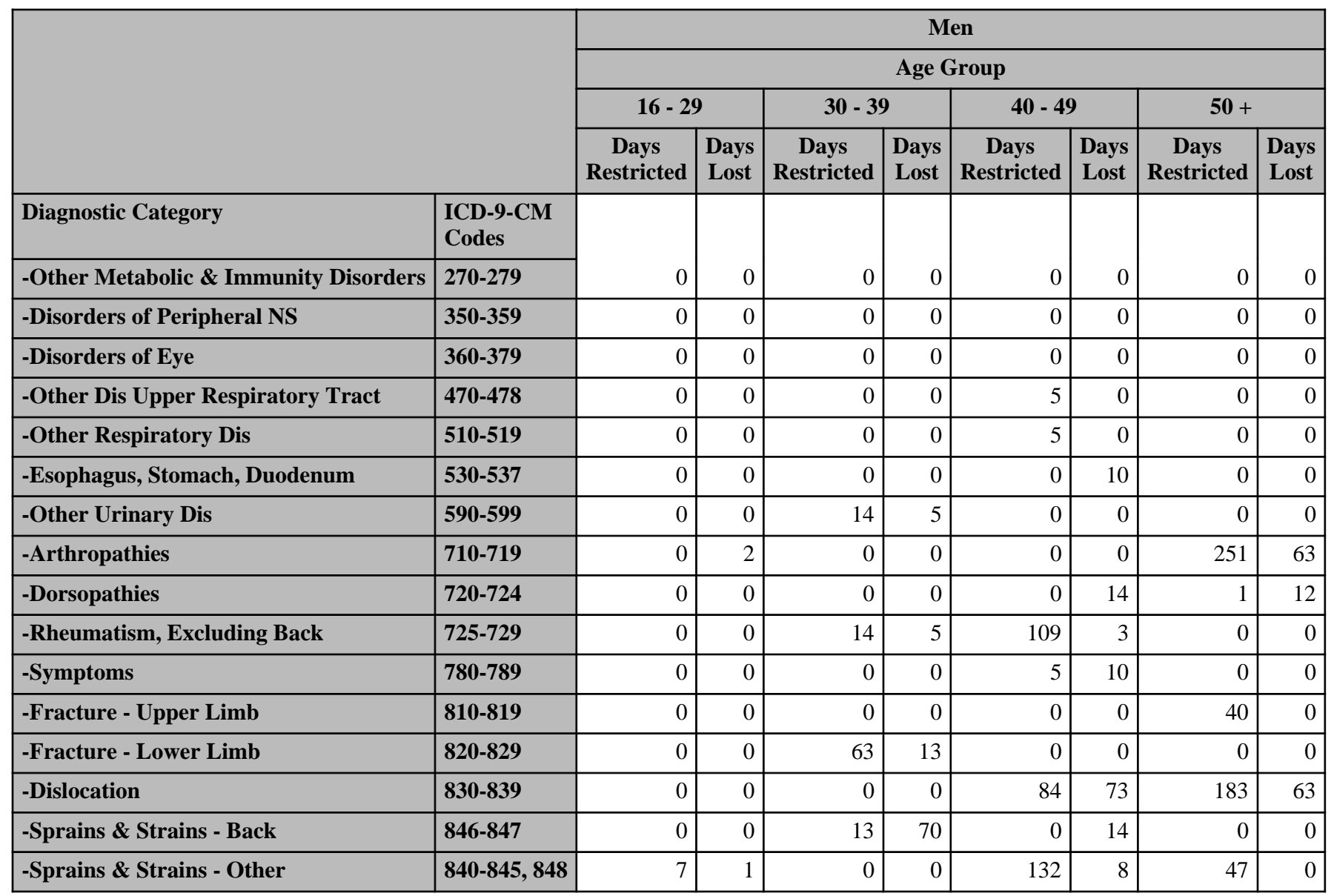

(Continued)

*OSHA events with >1 ICD-9-CM code in the same diagnostic category were counted only once. Only those diagnostic categories and gender/age combinations with at least one occurrence appear in this table. 


\section{Nevada Test Site 2006}

OSHA Data

Appendix P. Number of Workdays Lost or with Restricted Activity in Each Diagnostic Category by Gender and Age*

\begin{tabular}{|c|c|c|c|c|c|c|c|c|c|}
\hline & & \multicolumn{8}{|c|}{ Men } \\
\hline & & \multicolumn{8}{|c|}{ Age Group } \\
\hline & & \multicolumn{2}{|c|}{$16-29$} & \multicolumn{2}{|c|}{$30-39$} & \multicolumn{2}{|c|}{$40-49$} & \multicolumn{2}{|l|}{$50+$} \\
\hline Diagnostic Category & $\begin{array}{l}\text { ICD-9-CM } \\
\text { Codes }\end{array}$ & & & & & & & & \\
\hline -Open Wound - Upper Limb & 880-887 & 0 & 0 & 0 & 0 & 0 & 0 & 3 & 0 \\
\hline -Open Wound - Lower Limb & 890-897 & 0 & 0 & 170 & 10 & 0 & 0 & 0 & 0 \\
\hline -Superficial Injury & $910-919$ & 0 & 0 & 0 & 0 & 0 & 0 & 0 & 0 \\
\hline -Contusion & $920-924$ & 0 & 2 & 0 & 0 & 0 & 0 & 0 & 0 \\
\hline -Unspecified Effects - External Causes & 990-995 & 0 & 0 & 0 & 0 & 0 & 10 & 0 & 0 \\
\hline
\end{tabular}

*OSHA events with >1 ICD-9-CM code in the same diagnostic category were counted only once. Only those diagnostic categories and gender/age combinations with at least one occurrence appear in this table. 
Nevada Test Site 2006

OSHA Data

Appendix Q. Number of Occurrences in Each Accident Category by Gender and Age*

\begin{tabular}{|c|c|c|c|c|c|c|c|c|c|c|c|}
\hline & & \multicolumn{4}{|c|}{ Women } & \multicolumn{5}{|c|}{ Men } & \multirow[b]{3}{*}{ TOTAL } \\
\hline & & \multicolumn{3}{|c|}{ Age Group } & \multirow[b]{2}{*}{ TOTAL } & \multicolumn{4}{|c|}{ Age Group } & \multirow[b]{2}{*}{ TOTAL } & \\
\hline & & $30-39$ & 40 - 49 & $50+$ & & $16-29$ & 30 - 39 & $40-49$ & $50+$ & & \\
\hline Type of Accident & E Codes & & & & & & & & & & \\
\hline Motor Vehicle Nontraffic & E820-E825 & 0 & 0 & 0 & 0 & 0 & 0 & 0 & 2 & 2 & 2 \\
\hline Falls & \begin{tabular}{|l|} 
E880-E888 \\
\end{tabular} & 0 & 1 & 2 & 3 & 1 & 1 & 2 & 4 & 8 & 11 \\
\hline Natural/Environmental Factors & \begin{tabular}{|l|} 
E900-E909 \\
\end{tabular} & 0 & 0 & 0 & 0 & 0 & 0 & 4 & 0 & 4 & 4 \\
\hline Submersion/Suffocation/Foreign Bodies & E910-E915 & 0 & 1 & 0 & 1 & 0 & 1 & 1 & 0 & 2 & 3 \\
\hline Other Accidents & E916-E928 & 3 & 3 & 4 & 10 & 6 & 6 & 11 & 16 & 39 & 49 \\
\hline
\end{tabular}

*Only those accident types and gender/age combinations with at least one occurrence appear in this table.

Appendix R. Number of Workdays Lost or with Restricted Activity in Each Accident Category by Gender and Age*

\begin{tabular}{|c|c|c|c|c|c|c|c|}
\hline & & \multicolumn{6}{|c|}{ Women } \\
\hline & & \multicolumn{6}{|c|}{ Age Group } \\
\hline & & \multicolumn{2}{|c|}{30 - 39} & \multicolumn{2}{|c|}{$40-49$} & \multicolumn{2}{|l|}{$50+$} \\
\hline & & $\begin{array}{c}\text { Days } \\
\text { Restricted }\end{array}$ & $\begin{array}{l}\text { Days } \\
\text { Lost }\end{array}$ & $\begin{array}{c}\text { Days } \\
\text { Restricted }\end{array}$ & $\begin{array}{l}\text { Days } \\
\text { Lost }\end{array}$ & $\begin{array}{c}\text { Days } \\
\text { Restricted }\end{array}$ & $\begin{array}{l}\text { Days } \\
\text { Lost }\end{array}$ \\
\hline Type of Accident & E Codes & \multirow[b]{2}{*}{0} & \multirow[b]{2}{*}{0} & \multirow[b]{2}{*}{0} & \multirow[b]{2}{*}{0} & \multirow[b]{2}{*}{0} & \multirow[b]{2}{*}{0} \\
\hline Falls & E880-E888 & & & & & & \\
\hline Submersion/Suffocation/Foreign Bodies & E910-E915 & 0 & 0 & 0 & 0 & 0 & 0 \\
\hline Other Accidents & E916-E928 & 0 & 0 & 14 & 136 & 0 & 0 \\
\hline
\end{tabular}

\begin{tabular}{|c|c|c|c|c|c|c|c|c|c|}
\hline & & \multicolumn{8}{|c|}{ Men } \\
\hline & & \multicolumn{8}{|c|}{ Age Group } \\
\hline & & \multicolumn{2}{|c|}{$16-29$} & \multicolumn{2}{|c|}{$30-39$} & \multicolumn{2}{|c|}{$40-49$} & \multicolumn{2}{|l|}{$50+$} \\
\hline & & $\begin{array}{c}\text { Days } \\
\text { Restricted }\end{array}$ & $\begin{array}{l}\text { Days } \\
\text { Lost }\end{array}$ & $\begin{array}{c}\text { Days } \\
\text { Restricted }\end{array}$ & $\begin{array}{l}\text { Days } \\
\text { Lost }\end{array}$ & $\begin{array}{c}\text { Days } \\
\text { Restricted }\end{array}$ & $\begin{array}{l}\text { Days } \\
\text { Lost }\end{array}$ & $\begin{array}{c}\text { Days } \\
\text { Restricted }\end{array}$ & $\begin{array}{l}\text { Days } \\
\text { Lost }\end{array}$ \\
\hline Type of Accident & E Codes & \multirow[b]{2}{*}{0} & \multirow[b]{2}{*}{0} & \multirow[b]{2}{*}{0} & \multirow[b]{2}{*}{0} & \multirow[b]{2}{*}{0} & \multirow[b]{2}{*}{0} & \multirow[b]{2}{*}{0} & \multirow[b]{2}{*}{0} \\
\hline Motor Vehicle Nontraffic & E820-E825 & & & & & & & & \\
\hline Falls & E880-E888 & 7 & 1 & 63 & 13 & 38 & 66 & 118 & 0 \\
\hline Natural/Environmental Factors & E900-E909 & 0 & 0 & 0 & 0 & 0 & 10 & 0 & 0 \\
\hline Submersion/Suffocation/Foreign Bodies & E910-E915 & 0 & 0 & 0 & 0 & 5 & 0 & 0 & 0 \\
\hline Other Accidents & E916-E928 & 0 & 2 & 197 & 85 & 308 & 150 & 309 & 226 \\
\hline
\end{tabular}

*OSHA events with $>1 \mathrm{E}$ code in the same accident type were counted only once. Only those accident types and gender/age combinations with at least one occurrence appear in this table. 


\section{Nevada Test Site 2006}

OSHA Data

Appendix S. Number of Diagnoses in Each Diagnostic Category by Gender and Job Category*

\begin{tabular}{|c|c|c|c|c|c|c|}
\hline & & \multicolumn{5}{|c|}{ Women } \\
\hline & & \multicolumn{4}{|c|}{ Job Category } & \multirow[b]{2}{*}{ TOTAL } \\
\hline & & Professional & $\begin{array}{l}\text { Technical } \\
\text { Support }\end{array}$ & $\begin{array}{c}\text { Security } \\
\text { and } \\
\text { Fire }\end{array}$ & Crafts & \\
\hline Diagnostic Category & $\begin{array}{l}\text { ICD-9-CM } \\
\text { Code }\end{array}$ & & & & & \\
\hline MUSCULOSKELETAL \& CONNECTIVE TISSUE & 710-739 & 2 & 2 & 1 & 2 & 7 \\
\hline -Arthropathies & $710-719$ & 2 & 2 & 1 & 0 & 5 \\
\hline -Dorsopathies & $720-724$ & 0 & 0 & 0 & 2 & 2 \\
\hline SYMPTOMS, SIGNS, \& ILL-DEFINED CONDITIONS & $780-799$ & 0 & 0 & 0 & 3 & 3 \\
\hline -Symptoms & 780-789 & 0 & 0 & 0 & 3 & 3 \\
\hline INJURY \& POISONING & 800-999 & 1 & 1 & 1 & 12 & 15 \\
\hline -Fracture - Upper Limb & 810-819 & 0 & 0 & 0 & 1 & 1 \\
\hline -Sprains \& Strains - Back & 846-847 & 0 & 0 & 0 & 1 & 1 \\
\hline -Sprains \& Strains - Other & $840-845,848$ & 0 & 0 & 1 & 2 & 3 \\
\hline -Open Wound - Head, Neck, Trunk & $870-879$ & 0 & 1 & 0 & 1 & 2 \\
\hline -Contusion & $920-924$ & 0 & 0 & 0 & 4 & 4 \\
\hline -Foreign Body Entering Orifice & 930-939 & 1 & 0 & 0 & 0 & 1 \\
\hline -Burns & $940-949$ & 0 & 0 & 0 & 1 & 1 \\
\hline -Complications \& Unspecified Injuries & 958-959 & 0 & 0 & 0 & 2 & 2 \\
\hline
\end{tabular}

\begin{tabular}{|c|c|c|c|c|c|}
\hline & \multicolumn{5}{|c|}{ Women } \\
\hline & \multicolumn{4}{|c|}{ Job Category } & \multirow[b]{2}{*}{ TOTAL } \\
\hline & Professional & $\begin{array}{l}\text { Technical } \\
\text { Support }\end{array}$ & $\begin{array}{c}\text { Security } \\
\text { and } \\
\text { Fire }\end{array}$ & Crafts & \\
\hline $\begin{array}{l}\text { Diagnostic } \\
\text { Category }\end{array}$ & \multirow[b]{2}{*}{3} & \multirow[b]{2}{*}{3} & \multirow[b]{2}{*}{2} & \multirow[b]{2}{*}{17} & \multirow[b]{2}{*}{25} \\
\hline Total & & & & & \\
\hline
\end{tabular}

\begin{tabular}{|c|c|c|c|c|c|c|}
\hline & & \multicolumn{5}{|c|}{ Men } \\
\hline & & \multicolumn{4}{|c|}{ Job Category } & \multirow[b]{2}{*}{ TOTAL } \\
\hline & & Professional & $\begin{array}{l}\text { Administrative } \\
\text { Support }\end{array}$ & $\begin{array}{c}\text { Security } \\
\text { and } \\
\text { Fire }\end{array}$ & Crafts & \\
\hline Diagnostic Category & $\begin{array}{l}\text { ICD-9-CM } \\
\text { Code }\end{array}$ & \multirow[b]{2}{*}{0} & \multirow[b]{2}{*}{0} & \multirow[b]{2}{*}{1} & \multirow[b]{2}{*}{0} & \multirow[b]{2}{*}{1} \\
\hline ENDOCRINE/METABOLIC/IMMUNITY & $240-279$ & & & & & \\
\hline -Other Metabolic \& Immunity Disorders & 270-279 & 0 & 0 & 1 & 0 & 1 \\
\hline NERVOUS SYSTEM (NS) \& SENSE ORGANS & 320-389 & 1 & 0 & 4 & 0 & 5 \\
\hline -Disorders of Peripheral NS & $350-359$ & 1 & 0 & 1 & 0 & 2 \\
\hline -Disorders of Eye & $360-379$ & 0 & 0 & 3 & 0 & 3 \\
\hline RESPIRATORY SYSTEM & $460-519$ & 0 & 0 & 3 & 0 & 3 \\
\hline
\end{tabular}

(Continued)

*Only those diagnostic categories and gender/job category combinations with at least one occurrence appear in this table. 


\section{Nevada Test Site 2006}

OSHA Data

Appendix S. Number of Diagnoses in Each Diagnostic Category by Gender and Job Category*

\begin{tabular}{|c|c|c|c|c|c|c|}
\hline & & \multicolumn{5}{|c|}{ Men } \\
\hline & & \multicolumn{4}{|c|}{ Job Category } & \multirow[b]{2}{*}{ TOTAL } \\
\hline & & Professional & $\begin{array}{c}\text { Administrative } \\
\text { Support }\end{array}$ & $\begin{array}{c}\text { Security } \\
\text { and } \\
\text { Fire }\end{array}$ & Crafts & \\
\hline Diagnostic Category & $\begin{array}{l}\text { ICD-9-CM } \\
\text { Code }\end{array}$ & \multirow[b]{2}{*}{0} & \multirow[b]{2}{*}{0} & \multirow[b]{2}{*}{2} & \multirow[b]{2}{*}{0} & \multirow[b]{2}{*}{2} \\
\hline -Other Dis Upper Respiratory Tract & $470-478$ & & & & & \\
\hline -Other Respiratory Dis & $510-519$ & 0 & 0 & 1 & 0 & 1 \\
\hline DIGESTIVE SYSTEM & $520-579$ & 0 & 0 & 1 & 0 & 1 \\
\hline -Esophagus, Stomach, Duodenum & $530-537$ & 0 & 0 & 1 & 0 & 1 \\
\hline GENITOURINARY SYSTEM & $580-629$ & 0 & 0 & 1 & 0 & 1 \\
\hline -Other Urinary Dis & $590-599$ & 0 & 0 & 1 & 0 & 1 \\
\hline MUSCULOSKELETAL \& CONNECTIVE TISSUE & $710-739$ & 5 & 1 & 17 & 6 & 29 \\
\hline -Arthropathies & $710-719$ & 2 & 1 & 6 & 2 & 11 \\
\hline -Dorsopathies & $720-724$ & 3 & 0 & 6 & 2 & 11 \\
\hline -Rheumatism, Excluding Back & 725-729 & 0 & 0 & 5 & 2 & 7 \\
\hline SYMPTOMS, SIGNS, \& ILL-DEFINED CONDITIONS & $780-799$ & 1 & 0 & 5 & 3 & 9 \\
\hline -Symptoms & 780-789 & 1 & 0 & 5 & 3 & 9 \\
\hline INJURY \& POISONING & $800-999$ & 5 & 2 & 16 & 26 & 49 \\
\hline -Fracture - Upper Limb & 810-819 & 0 & 0 & 0 & 1 & 1 \\
\hline -Fracture - Lower Limb & $820-829$ & 0 & 0 & 0 & 1 & 1 \\
\hline -Dislocation & $830-839$ & 0 & 1 & 1 & 3 & 5 \\
\hline -Sprains \& Strains - Back & 846-847 & 0 & 0 & 2 & 1 & 3 \\
\hline -Sprains \& Strains - Other & $840-845,848$ & 1 & 0 & 4 & 2 & 7 \\
\hline -Open Wound - Head, Neck, Trunk & $870-879$ & 1 & 0 & 1 & 0 & 2 \\
\hline -Open Wound - Upper Limb & $880-887$ & 0 & 1 & 0 & 2 & 3 \\
\hline -Open Wound - Lower Limb & 890-897 & 0 & 0 & 1 & 1 & 2 \\
\hline -Superficial Injury & 910-919 & 0 & 0 & 1 & 2 & 3 \\
\hline -Contusion & $920-924$ & 0 & 0 & 1 & 0 & 1 \\
\hline -Foreign Body Entering Orifice & 930-939 & 0 & 0 & 2 & 0 & 2 \\
\hline -Complications \& Unspecified Injuries & 958-959 & 3 & 0 & 2 & 13 & 18 \\
\hline -Unspecified Effects - External Causes & $990-995$ & 0 & 0 & 1 & 0 & 1 \\
\hline
\end{tabular}

\begin{tabular}{|l|r|r|r|r|r|}
\hline \multirow{4}{*}{} & \multicolumn{4}{|c|}{ Men } & \\
\cline { 2 - 5 } & Professional & $\begin{array}{c}\text { Administrative } \\
\text { Support }\end{array}$ & $\begin{array}{c}\text { Security } \\
\text { and } \\
\text { Fire }\end{array}$ & Crafts & \multirow{2}{*}{ TOTAL } \\
\hline $\begin{array}{l}\text { Diagnostic } \\
\text { Category }\end{array}$ & & & & & \\
\cline { 4 - 6 } Total & 12 & & 48 & 35 & 98 \\
\hline
\end{tabular}

*Only those diagnostic categories and gender/job category combinations with at least one occurrence appear in this table. 


\section{Nevada Test Site 2006}

OSHA Data

Appendix T. Number of Workdays Lost or with Restricted Activity in Each Diagnostic Category by Gender and Job Category*

\begin{tabular}{|c|c|c|c|c|c|c|c|c|c|}
\hline & & \multicolumn{8}{|c|}{ Women } \\
\hline & & \multicolumn{8}{|c|}{ Job Category } \\
\hline & & \multicolumn{2}{|c|}{ Professional } & \multicolumn{2}{|c|}{$\begin{array}{l}\text { Technical } \\
\text { Support }\end{array}$} & \multicolumn{2}{|c|}{$\begin{array}{c}\text { Security and } \\
\text { Fire }\end{array}$} & \multicolumn{2}{|c|}{ Crafts } \\
\hline & & \begin{tabular}{c|} 
Days \\
Restricted
\end{tabular} & $\begin{array}{l}\text { Days } \\
\text { Lost }\end{array}$ & $\begin{array}{c}\text { Days } \\
\text { Restricted }\end{array}$ & \begin{tabular}{|l|} 
Days \\
Lost
\end{tabular} & $\begin{array}{c}\text { Days } \\
\text { Restricted }\end{array}$ & \begin{tabular}{|l|} 
Days \\
Lost
\end{tabular} & \begin{tabular}{|c|} 
Days \\
Restricted
\end{tabular} & $\begin{array}{l}\text { Days } \\
\text { Lost }\end{array}$ \\
\hline Diagnostic Category & \begin{tabular}{|l|} 
ICD-9-CM \\
Codes
\end{tabular} & & & & & & & & \\
\hline -Arthropathies & $710-719$ & 0 & 0 & 0 & 0 & 7 & 12 & 0 & 0 \\
\hline -Dorsopathies & $720-724$ & 0 & 0 & 0 & 0 & 0 & 0 & 0 & 0 \\
\hline -Symptoms & $780-789$ & 0 & 0 & 0 & 0 & 0 & 0 & 0 & 0 \\
\hline -Fracture - Upper Limb & 810-819 & 0 & 0 & 0 & 0 & 0 & 0 & 0 & 0 \\
\hline -Sprains \& Strains - Back & 846-847 & 0 & 0 & 0 & 0 & 0 & 0 & 0 & 0 \\
\hline -Sprains \& Strains - Other & $840-845,848$ & 0 & 0 & 0 & 0 & 7 & 12 & 7 & 124 \\
\hline -Open Wound - Head, Neck, Trunk & $870-879$ & 0 & 0 & 0 & 0 & 0 & 0 & 0 & 0 \\
\hline -Contusion & $920-924$ & 0 & 0 & 0 & 0 & 0 & 0 & 7 & 124 \\
\hline -Foreign Body Entering Orifice & 930-939 & 0 & 0 & 0 & 0 & 0 & 0 & 0 & 0 \\
\hline -Burns & 940-949 & 0 & 0 & 0 & 0 & 0 & 0 & 0 & 0 \\
\hline -Complications \& Unspecified Injuries & 958-959 & 0 & 0 & 0 & 0 & 0 & 0 & 0 & 0 \\
\hline
\end{tabular}

\begin{tabular}{|c|c|c|c|c|c|c|c|c|c|}
\hline & & \multicolumn{8}{|c|}{ Men } \\
\hline & & \multicolumn{8}{|c|}{ Job Category } \\
\hline & & \multicolumn{2}{|c|}{ Professional } & \multicolumn{2}{|c|}{$\begin{array}{l}\text { Administrative } \\
\text { Support }\end{array}$} & \multicolumn{2}{|c|}{$\begin{array}{c}\text { Security and } \\
\text { Fire }\end{array}$} & \multicolumn{2}{|c|}{ Crafts } \\
\hline & & $\begin{array}{c}\text { Days } \\
\text { Restricted }\end{array}$ & $\begin{array}{l}\text { Days } \\
\text { Lost }\end{array}$ & \begin{tabular}{|c|} 
Days \\
Restricted
\end{tabular} & $\begin{array}{l}\text { Days } \\
\text { Lost }\end{array}$ & $\begin{array}{c}\text { Days } \\
\text { Restricted }\end{array}$ & $\begin{array}{l}\text { Days } \\
\text { Lost }\end{array}$ & $\begin{array}{c}\text { Days } \\
\text { Restricted }\end{array}$ & $\begin{array}{l}\text { Days } \\
\text { Lost }\end{array}$ \\
\hline Diagnostic Category & $\begin{array}{l}\text { ICD-9-CM } \\
\text { Codes }\end{array}$ & & & & & & & & \\
\hline -Other Metabolic \& Immunity Disorders & $270-279$ & 0 & 0 & 0 & 0 & 0 & 0 & 0 & 0 \\
\hline -Disorders of Peripheral NS & $350-359$ & 0 & 0 & 0 & 0 & 0 & 0 & 0 & 0 \\
\hline -Disorders of Eye & $360-379$ & 0 & 0 & 0 & 0 & 0 & 0 & 0 & 0 \\
\hline -Other Dis Upper Respiratory Tract & $470-478$ & 0 & 0 & 0 & 0 & 5 & 0 & 0 & 0 \\
\hline -Other Respiratory Dis & $510-519$ & 0 & 0 & 0 & 0 & 5 & 0 & 0 & 0 \\
\hline -Esophagus, Stomach, Duodenum & $530-537$ & 0 & 0 & 0 & 0 & 0 & 10 & 0 & 0 \\
\hline -Other Urinary Dis & $590-599$ & 0 & 0 & 0 & 0 & 14 & 5 & 0 & 0 \\
\hline -Arthropathies & $710-719$ & 0 & 0 & 0 & 26 & 143 & 39 & 108 & 0 \\
\hline -Dorsopathies & $720-724$ & 0 & 0 & 0 & 0 & 0 & 14 & 1 & 12 \\
\hline -Rheumatism, Excluding Back & $725-729$ & 0 & 0 & 0 & 0 & 123 & 8 & 0 & $\overline{0}$ \\
\hline -Symptoms & 780-789 & 0 & 0 & 0 & 0 & 5 & 10 & 0 & 0 \\
\hline -Fracture - Upper Limb & $810-819$ & 0 & 0 & 0 & 0 & 0 & 0 & 40 & 0 \\
\hline -Fracture - Lower Limb & $820-829$ & 0 & 0 & 0 & 0 & 0 & 0 & 63 & 13 \\
\hline -Dislocation & $830-839$ & 0 & 0 & 0 & 26 & 143 & 37 & 124 & 73 \\
\hline
\end{tabular}

(Continued)

*OSHA events with >1 ICD-9-CM code in the same diagnostic category were counted only once. Only those diagnostic categories and gender/job category combinations with at least one occurrence appear in this table. 


\section{Nevada Test Site 2006}

OSHA Data

Appendix T. Number of Workdays Lost or with Restricted Activity in Each Diagnostic Category by Gender and Job Category*

\begin{tabular}{|c|c|c|c|c|c|c|c|c|c|}
\hline & & \multicolumn{8}{|c|}{ Men } \\
\hline & & \multicolumn{8}{|c|}{ Job Category } \\
\hline & & \multicolumn{2}{|c|}{ Professional } & \multicolumn{2}{|c|}{$\begin{array}{l}\text { Administrative } \\
\text { Support }\end{array}$} & \multicolumn{2}{|c|}{$\begin{array}{c}\text { Security and } \\
\text { Fire }\end{array}$} & \multicolumn{2}{|c|}{ Crafts } \\
\hline & & $\begin{array}{c}\text { Days } \\
\text { Restricted }\end{array}$ & $\begin{array}{l}\text { Days } \\
\text { Lost }\end{array}$ & \begin{tabular}{|c|} 
Days \\
Restricted
\end{tabular} & $\begin{array}{l}\text { Days } \\
\text { Lost }\end{array}$ & $\begin{array}{c}\text { Days } \\
\text { Restricted }\end{array}$ & $\begin{array}{l}\text { Days } \\
\text { Lost }\end{array}$ & $\begin{array}{c}\text { Days } \\
\text { Restricted }\end{array}$ & $\begin{array}{l}\text { Days } \\
\text { Lost }\end{array}$ \\
\hline Diagnostic Category & $\begin{array}{l}\text { ICD-9-CM } \\
\text { Codes }\end{array}$ & & & & & & & & \\
\hline -Sprains \& Strains - Back & 846-847 & 0 & 0 & 0 & 0 & 0 & 14 & 13 & 70 \\
\hline -Sprains \& Strains - Other & $840-845,848$ & 7 & 0 & 0 & 0 & 139 & 9 & 40 & 0 \\
\hline -Open Wound - Head, Neck, Trunk & 870-879 & 0 & 0 & 0 & 0 & 0 & 0 & 0 & 0 \\
\hline -Open Wound - Upper Limb & 880-887 & 0 & 0 & 0 & 0 & 0 & 0 & 3 & 0 \\
\hline -Open Wound - Lower Limb & $890-897$ & 0 & 0 & 0 & 0 & 170 & 10 & 0 & 0 \\
\hline -Superficial Injury & $910-919$ & 0 & 0 & 0 & 0 & 0 & 0 & 0 & 0 \\
\hline -Contusion & $920-924$ & 0 & 0 & 0 & 0 & 0 & 2 & 0 & 0 \\
\hline -Foreign Body Entering Orifice & 930-939 & 0 & 0 & 0 & 0 & 5 & 0 & 0 & 0 \\
\hline -Complications \& Unspecified Injuries & 958-959 & 0 & 0 & 0 & 0 & 130 & 16 & 125 & 256 \\
\hline -Unspecified Effects - External Causes & $990-995$ & 0 & 0 & 0 & 0 & 0 & 10 & 0 & 0 \\
\hline
\end{tabular}

*OSHA events with >1 ICD-9-CM code in the same diagnostic category were counted only once. Only those diagnostic categories and gender/job category combinations with at least one occurrence appear in this table. 


\section{Nevada Test Site 2006}

OSHA Data

Appendix U. Number of Occurrences in Each Accident Category by Gender and Job Category*

\begin{tabular}{|c|c|c|c|c|c|c|}
\hline & & \multicolumn{5}{|c|}{ Women } \\
\hline & & \multicolumn{4}{|c|}{ Job Category } & \multirow[b]{2}{*}{ TOTAL } \\
\hline & & Professional & $\begin{array}{c}\text { Technical } \\
\text { Support }\end{array}$ & $\begin{array}{c}\text { Security } \\
\text { and } \\
\text { Fire }\end{array}$ & Crafts & \\
\hline Type of Accident & E CODES & \multirow[b]{2}{*}{0} & \multirow[b]{2}{*}{1} & \multirow[b]{2}{*}{0} & \multirow[b]{2}{*}{2} & \multirow[b]{2}{*}{3} \\
\hline Falls & E880-E888 & & & & & \\
\hline Submersion/Suffocation/Foreign Bodies & E910-E915 & 1 & 0 & 0 & 0 & 1 \\
\hline Other Accidents & E916-E928 & 1 & 2 & 1 & 6 & 10 \\
\hline
\end{tabular}

\begin{tabular}{|c|c|c|c|c|c|c|}
\hline & & \multicolumn{5}{|c|}{ Men } \\
\hline & & \multicolumn{4}{|c|}{ Job Category } & \multirow[b]{2}{*}{ TOTAL } \\
\hline & & Professional & $\begin{array}{l}\text { Administrative } \\
\text { Support }\end{array}$ & $\begin{array}{c}\text { Security } \\
\text { and } \\
\text { Fire }\end{array}$ & Crafts & \\
\hline Type of Accident & E CODES & \multirow[b]{2}{*}{0} & \multirow[b]{2}{*}{0} & \multirow[b]{2}{*}{0} & \multirow[b]{2}{*}{2} & \multirow[b]{2}{*}{2} \\
\hline Motor Vehicle Nontraffic & E820-E825 & & & & & \\
\hline Falls & E880-E888 & 0 & 0 & 1 & 7 & 8 \\
\hline Natural/Environmental Factors & E900-E909 & 0 & 0 & 2 & 2 & 4 \\
\hline Submersion/Suffocation/Foreign Bodies & E910-E915 & 0 & 0 & 2 & 0 & 2 \\
\hline Other Accidents & E916-E928 & 6 & 2 & 14 & 17 & 39 \\
\hline
\end{tabular}

*Only those accident types and gender/job category combinations with at least one occurrence appear in this table. 


\section{Nevada Test Site 2006}

OSHA Data

Appendix V. Number of Workdays Lost or with Restricted Activity in Each Accident Category by Gender and Job Category*

\begin{tabular}{|c|c|c|c|c|c|c|c|c|c|}
\hline & & \multicolumn{8}{|c|}{ Women } \\
\hline & & \multicolumn{8}{|c|}{ Job Category } \\
\hline & & \multicolumn{2}{|c|}{ Professional } & \multicolumn{2}{|c|}{$\begin{array}{l}\text { Technical } \\
\text { Support }\end{array}$} & \multicolumn{2}{|c|}{$\begin{array}{c}\text { Security and } \\
\text { Fire }\end{array}$} & \multicolumn{2}{|c|}{ Crafts } \\
\hline & & $\begin{array}{c}\text { Days } \\
\text { Restricted }\end{array}$ & $\begin{array}{l}\text { Days } \\
\text { Lost }\end{array}$ & $\begin{array}{c}\text { Days } \\
\text { Restricted }\end{array}$ & \begin{tabular}{|l|} 
Days \\
Lost
\end{tabular} & $\begin{array}{c}\text { Days } \\
\text { Restricted }\end{array}$ & \begin{tabular}{|l|} 
Days \\
Lost
\end{tabular} & $\begin{array}{c}\text { Days } \\
\text { Restricted }\end{array}$ & \begin{tabular}{|l} 
Days \\
Lost
\end{tabular} \\
\hline Type of Accident & E Codes & & & & & & & & \\
\hline Falls & E880-E888 & 0 & 0 & 0 & 0 & 0 & 0 & 0 & 0 \\
\hline Submersion/Suffocation/Foreign Bodies & E910-E915 & 0 & 0 & 0 & 0 & 0 & 0 & 0 & 0 \\
\hline Other Accidents & E916-E928 & 0 & 0 & 0 & 0 & 7 & 12 & 7 & 124 \\
\hline
\end{tabular}

\begin{tabular}{|c|c|c|c|c|c|c|c|c|c|}
\hline & & \multicolumn{8}{|c|}{ Men } \\
\hline & & \multicolumn{8}{|c|}{ Job Category } \\
\hline & & \multicolumn{2}{|c|}{ Professional } & \multicolumn{2}{|c|}{$\begin{array}{l}\text { Administrative } \\
\text { Support }\end{array}$} & \multicolumn{2}{|c|}{$\begin{array}{l}\text { Security and } \\
\text { Fire }\end{array}$} & \multicolumn{2}{|c|}{ Crafts } \\
\hline & & $\begin{array}{c}\text { Days } \\
\text { Restricted }\end{array}$ & $\begin{array}{l}\text { Days } \\
\text { Lost }\end{array}$ & \begin{tabular}{|c|} 
Days \\
Restricted
\end{tabular} & $\begin{array}{l}\text { Days } \\
\text { Lost }\end{array}$ & $\begin{array}{c}\text { Days } \\
\text { Restricted }\end{array}$ & \begin{tabular}{|l|} 
Days \\
Lost
\end{tabular} & $\begin{array}{c}\text { Days } \\
\text { Restricted }\end{array}$ & $\begin{array}{l}\text { Days } \\
\text { Lost }\end{array}$ \\
\hline Falls & E880-E888 & 0 & 0 & 0 & 0 & 7 & 1 & 219 & 79 \\
\hline Natural/Environmental Factors & E900-E909 & 0 & 0 & 0 & 0 & 0 & 10 & 0 & 0 \\
\hline Submersion/Suffocation/Foreign Bodies & E910-E915 & 0 & 0 & 0 & 0 & 5 & 0 & 0 & 0 \\
\hline Other Accidents & E916-E928 & 7 & 0 & 0 & 26 & 589 & 92 & 218 & 345 \\
\hline
\end{tabular}

*OSHA events with >1 E code in the same accident type were counted only once. Only those accident types and gender/job category combinations with at least one occurrence appear in this table. 
Nevada Test Site 2006

OSHA Data

Appendix W. Age-Adjusted OSHA Illness and Injury Rates by Diagnostic Category

Part 1. Men

\begin{tabular}{|c|c|c|c|c|c|}
\hline Category of Diagnoses & ICD-9-CM Code & $\begin{array}{l}\text { Number of } \\
\text { Diagnoses }\end{array}$ & $\begin{array}{c}\text { Age-Adjusted } \\
\text { Rate per 1000* }\end{array}$ & \begin{tabular}{|c|} 
Lower $95 \%$ \\
Confidence \\
Limit per 1000
\end{tabular} & \begin{tabular}{|c|} 
Upper $95 \%$ \\
Confidence \\
Limit per 1000
\end{tabular} \\
\hline Infections/Parasites & $001-139$ & 0 & 0 & 0 & 0 \\
\hline Cancer & $140-208,230-234$ & 0 & 0 & 0 & 0 \\
\hline Benign Growths & $210-229,235-239$ & 0 & 0 & 0 & 0 \\
\hline Endocrine/Metabolic & $240-279$ & 1 & 0.7 & 0.1 & 5.0 \\
\hline Blood & $280-289$ & 0 & 0 & 0 & 0 \\
\hline Mental & $290-319$ & 0 & 0 & 0 & 0 \\
\hline Nervous System & $320-389$ & 5 & 2.8 & 1.1 & 7.0 \\
\hline -Disorders of Peripheral NS & $350-359$ & 2 & 1.0 & 0.2 & 4.5 \\
\hline -Disorders of Eye & $360-379$ & 3 & 1.8 & 0.6 & 5.7 \\
\hline -Disorders of Ear \& Mastoid & $380-389$ & 0 & 0 & 0 & 0 \\
\hline Heart/Circulatory & $390-459$ & 0 & 0 & 0 & 0 \\
\hline Respiratory & $460-519$ & 3 & 0.9 & 0.3 & 2.7 \\
\hline -Chronic Obstructive Diseases & $490-496$ & 0 & 0 & 0 & 0 \\
\hline -Lung Disease from External Agents & $500-508$ & 0 & 0 & 0 & 0 \\
\hline Digestive & $520-579$ & 1 & 0.3 & 0.0 & 2.1 \\
\hline -Hernias & $550-553$ & 0 & 0 & 0 & 0 \\
\hline Genitourinary & $580-629$ & 1 & 0.6 & 0.1 & 4.3 \\
\hline Skin & $680-709$ & 0 & 0 & 0 & 0 \\
\hline Musculoskeletal & $710-739$ & 29 & 10.6 & 7.0 & 16.0 \\
\hline -Arthropathies & $710-719$ & 11 & 3.3 & 1.6 & 6.7 \\
\hline -Dorsopathies & $720-724$ & 11 & 4.5 & 2.3 & 8.6 \\
\hline -Rheumatism, Excluding Back & $725-729$ & 7 & 2.8 & 1.2 & 6.3 \\
\hline Unspecified Symptoms & $780-799$ & 9 & 3.3 & 1.6 & 6.8 \\
\hline Injury & $800-999$ & 49 & 16.2 & 11.8 & 22.4 \\
\hline -Fractures - Skull, Neck, Trunk & $800-809$ & 0 & 0 & 0 & 0 \\
\hline -Fractures - Upper Limb & $810-819$ & 1 & 0.2 & 0.0 & 1.3 \\
\hline -Fractures - Lower Limb & $820-829$ & 1 & 0.6 & 0.1 & 4.3 \\
\hline -Dislocations & $830-839$ & 5 & 1.1 & 0.5 & 2.8 \\
\hline -Back Sprains \& Strains & $846-847$ & 3 & 1.2 & 0.4 & 4.0 \\
\hline -Other Sprains \& Strains & $840-845,848$ & 7 & 2.1 & 0.9 & 5.0 \\
\hline -Open Wounds - Head, Neck, Trunk & $870-879$ & 2 & 0.9 & 0.2 & 4.4 \\
\hline -Open Wounds - Upper Limb & $880-887$ & 3 & 1.1 & 0.3 & 4.2 \\
\hline -Open Wounds - Lower Limb & $890-897$ & 2 & 0.8 & 0.2 & 3.8 \\
\hline -Superficial Injuries & $910-919$ & 3 & 0.9 & 0.3 & 2.7 \\
\hline -Bruises & $920-924$ & 1 & 0.7 & 0.1 & 5.0 \\
\hline -Foreign Bodies Entering Orifice & $930-939$ & 2 & 0.9 & 0.2 & 3.9 \\
\hline -Burns & $940-949$ & 0 & 0 & 0 & 0 \\
\hline
\end{tabular}

*Standardized to age distribution of 2000 U.S. population. 
Nevada Test Site 2006

OSHA Data

Appendix W. Age-Adjusted OSHA Illness and Injury Rates by Diagnostic Category

\begin{tabular}{|r|r|r|r|r|}
\hline Category of Diagnoses & ICD-9-CM Code & $\begin{array}{c}\text { Number of } \\
\text { Diagnoses }\end{array}$ & $\begin{array}{r}\text { Lower 95\% } \\
\text { Age-Adjusted per 1000* } \\
\text { Rate ponfidence } \\
\text { Limit per 1000 }\end{array}$ & $\begin{array}{c}\text { Upper 95\% } \\
\text { Confidence } \\
\text { Limit per 1000 }\end{array}$ \\
\hline $\begin{array}{r}\text {-Adverse Reactions to Nonmedical } \\
\text { Substances or External Causes }\end{array}$ & & 1 & 0.3 & 0.0 \\
\hline -All Other Injuries & $\begin{array}{r}850-854,860-869,900-909, \\
925-929,950-979,996-999\end{array}$ & 18 & 5.4 & 3.2 \\
\hline Health Status/Health Service Contact & V01-V82 & 0 & 0.1 \\
\hline Total & & 98 & 0 & 35.4 \\
\hline
\end{tabular}

\section{Part 2. Women}

\begin{tabular}{|c|c|c|c|c|c|}
\hline Category of Diagnoses & ICD-9-CM Code & $\begin{array}{l}\text { Number of } \\
\text { Diagnoses }\end{array}$ & $\begin{array}{l}\text { Age-Adjusted } \\
\text { Rate per } 1000 *\end{array}$ & \begin{tabular}{|c|} 
Lower $95 \%$ \\
Confidence \\
Limit per 1000
\end{tabular} & $\begin{array}{c}\text { Upper } 95 \% \\
\text { Confidence } \\
\text { Limit per } 1000\end{array}$ \\
\hline Infections/Parasites & $001-139$ & 0 & 0 & 0 & 0 \\
\hline Cancer & $140-208,230-234$ & 0 & 0 & 0 & 0 \\
\hline Benign Growths & $210-229,235-239$ & 0 & 0 & 0 & 0 \\
\hline Endocrine/Metabolic & $240-279$ & 0 & 0 & 0 & 0 \\
\hline Blood & $280-289$ & 0 & 0 & 0 & 0 \\
\hline Mental & $290-319$ & 0 & 0 & 0 & 0 \\
\hline Nervous System & $320-389$ & 0 & 0 & 0 & 0 \\
\hline -Disorders of Peripheral NS & $350-359$ & 0 & 0 & 0 & 0 \\
\hline -Disorders of Eye & $360-379$ & 0 & 0 & 0 & 0 \\
\hline -Disorders of Ear \& Mastoid & $380-389$ & 0 & 0 & 0 & 0 \\
\hline Heart/Circulatory & $390-459$ & 0 & 0 & 0 & 0 \\
\hline Respiratory & $460-519$ & 0 & 0 & 0 & 0 \\
\hline -Chronic Obstructive Diseases & $490-496$ & 0 & 0 & 0 & 0 \\
\hline -Lung Disease from External Agents & $500-508$ & 0 & 0 & 0 & 0 \\
\hline Digestive & $520-579$ & 0 & 0 & 0 & 0 \\
\hline -Hernias & $550-553$ & 0 & 0 & 0 & 0 \\
\hline Genitourinary & $580-629$ & 0 & 0 & 0 & 0 \\
\hline Skin & $680-709$ & 0 & 0 & 0 & 0 \\
\hline Musculoskeletal & $710-739$ & 7 & 5.5 & 2.5 & 12.2 \\
\hline -Arthropathies & $710-719$ & 5 & 4.2 & 1.6 & 10.9 \\
\hline -Dorsopathies & $720-724$ & 2 & 1.3 & 0.3 & 5.1 \\
\hline -Rheumatism, Excluding Back & $725-729$ & 0 & 0 & 0 & 0 \\
\hline Unspecified Symptoms & $780-799$ & 3 & 2.5 & 0.8 & 7.8 \\
\hline Injury & $800-999$ & 15 & 10.2 & 5.9 & 17.5 \\
\hline -Fractures - Skull, Neck, Trunk & $800-809$ & 0 & 0 & 0 & 0 \\
\hline -Fractures - Upper Limb & $810-819$ & 1 & 0.5 & 0.1 & 3.5 \\
\hline -Fractures - Lower Limb & $820-829$ & 0 & 0 & 0 & 0 \\
\hline -Dislocations & $830-839$ & 0 & 0 & 0 & 0 \\
\hline
\end{tabular}

*Standardized to age distribution of 2000 U.S. population. 
Nevada Test Site 2006

OSHA Data

Appendix W. Age-Adjusted OSHA Illness and Injury Rates by Diagnostic Category

\begin{tabular}{|c|c|c|c|c|c|}
\hline Category of Diagnoses & ICD-9-CM Code & $\begin{array}{l}\text { Number of } \\
\text { Diagnoses }\end{array}$ & $\begin{array}{l}\text { Age-Adjusted } \\
\text { Rate per } 1000^{*}\end{array}$ & $\begin{array}{c}\text { Lower } 95 \% \\
\text { Confidence } \\
\text { Limit per } 1000\end{array}$ & $\begin{array}{c}\text { Upper } 95 \% \\
\text { Confidence } \\
\text { Limit per } 1000\end{array}$ \\
\hline -Back Sprains \& Strains & $846-847$ & 1 & 0.5 & 0.1 & 3.5 \\
\hline -Other Sprains \& Strains & $840-845,848$ & 3 & 1.8 & 0.6 & 5.6 \\
\hline -Open Wounds - Head, Neck, Trunk & $870-879$ & 2 & 1.0 & 0.2 & 4.0 \\
\hline -Open Wounds - Upper Limb & $880-887$ & 0 & 0 & 0 & 0 \\
\hline -Open Wounds - Lower Limb & $890-897$ & 0 & 0 & 0 & 0 \\
\hline -Superficial Injuries & $910-919$ & 0 & 0 & 0 & 0 \\
\hline -Bruises & $920-924$ & 4 & 2.7 & 1.0 & 7.4 \\
\hline -Foreign Bodies Entering Orifice & $930-939$ & 1 & 0.6 & 0.1 & 4.6 \\
\hline -Burns & $940-949$ & 1 & 1.3 & 0.2 & 9.1 \\
\hline $\begin{array}{l}\text {-Adverse Reactions to Nonmedical } \\
\text { Substances or External Causes }\end{array}$ & $980-995$ & 0 & 0 & 0 & 0 \\
\hline -All Other Injuries & \begin{tabular}{|c|}
$850-854,860-869,900-909$ \\
$925-929,950-979,996-999$
\end{tabular} & 2 & 1.8 & 0.4 & 8.1 \\
\hline Health Status/Health Service Contact & V01-V82 & 0 & 0 & 0 & 0 \\
\hline Total & & 25 & 18.1 & 11.9 & 27.6 \\
\hline
\end{tabular}

\section{Part 3. Men and Women}

\begin{tabular}{|c|c|c|c|c|c|}
\hline Category of Diagnoses & ICD-9-CM Code & $\begin{array}{l}\text { Number of } \\
\text { Diagnoses }\end{array}$ & $\begin{array}{l}\text { Age-Adjusted } \\
\text { Rate per } 1000 *\end{array}$ & $\begin{array}{c}\text { Lower } 95 \% \\
\text { Confidence } \\
\text { Limit per } 1000\end{array}$ & $\begin{array}{c}\text { Upper } 95 \% \\
\text { Confidence } \\
\text { Limit per } 1000\end{array}$ \\
\hline Infections/Parasites & 001-139 & 0 & 0 & 0 & 0 \\
\hline Cancer & $140-208,230-234$ & 0 & 0 & 0 & 0 \\
\hline Benign Growths & $210-229,235-239$ & 0 & 0 & 0 & 0 \\
\hline Endocrine/Metabolic & $240-279$ & 1 & 0.5 & 0.1 & 3.8 \\
\hline Blood & $280-289$ & 0 & 0 & 0 & 0 \\
\hline Mental & $290-319$ & 0 & 0 & 0 & 0 \\
\hline Nervous System & $320-389$ & 5 & 2.0 & 0.8 & 4.9 \\
\hline -Disorders of Peripheral NS & $350-359$ & 2 & 0.7 & 0.2 & 3.3 \\
\hline -Disorders of Eye & $360-379$ & 3 & 1.2 & 0.4 & 3.8 \\
\hline -Disorders of Ear \& Mastoid & $380-389$ & 0 & 0 & 0 & 0 \\
\hline Heart/Circulatory & $390-459$ & 0 & 0 & 0 & 0 \\
\hline Respiratory & $460-519$ & 3 & 0.6 & 0.2 & 1.9 \\
\hline -Chronic Obstructive Diseases & $490-496$ & 0 & 0 & 0 & 0 \\
\hline -Lung Disease from External Agents & $500-508$ & 0 & 0 & 0 & 0 \\
\hline Digestive & $520-579$ & 1 & 0.2 & 0.0 & 1.4 \\
\hline -Hernias & $550-553$ & 0 & 0 & 0 & 0 \\
\hline Genitourinary & $580-629$ & 1 & 0.4 & 0.1 & 2.9 \\
\hline Skin & $680-709$ & 0 & 0 & 0 & 0 \\
\hline Musculoskeletal & $710-739$ & 36 & 9.2 & 6.3 & 13.3 \\
\hline
\end{tabular}

*Standardized to age distribution of 2000 U.S. population. 
Nevada Test Site 2006

OSHA Data

Appendix W. Age-Adjusted OSHA Illness and Injury Rates by Diagnostic Category

\begin{tabular}{|c|c|c|c|c|c|}
\hline Category of Diagnoses & ICD-9-CM Code & $\begin{array}{l}\text { Number of } \\
\text { Diagnoses }\end{array}$ & $\begin{array}{l}\text { Age-Adjusted } \\
\text { Rate per 1000* }\end{array}$ & $\begin{array}{c}\text { Lower } 95 \% \\
\text { Confidence } \\
\text { Limit per } 1000\end{array}$ & $\begin{array}{c}\text { Upper } 95 \% \\
\text { Confidence } \\
\text { Limit per } 1000\end{array}$ \\
\hline -Arthropathies & $710-719$ & 16 & 3.7 & 2.1 & 6.6 \\
\hline -Dorsopathies & $720-724$ & 13 & 3.6 & 1.9 & 6.5 \\
\hline -Rheumatism, Excluding Back & $725-729$ & 7 & 1.9 & 0.8 & 4.3 \\
\hline Unspecified Symptoms & $780-799$ & 12 & 3.0 & 1.6 & 5.6 \\
\hline Injury & $800-999$ & 64 & 14.6 & 11.0 & 19.2 \\
\hline -Fractures - Skull, Neck, Trunk & $800-809$ & 0 & 0 & 0 & 0 \\
\hline -Fractures - Upper Limb & $810-819$ & 2 & 0.3 & 0.1 & 1.1 \\
\hline -Fractures - Lower Limb & $820-829$ & 1 & 0.4 & 0.1 & 2.9 \\
\hline -Dislocations & $830-839$ & 5 & 0.8 & 0.3 & 2.0 \\
\hline -Back Sprains \& Strains & $846-847$ & 4 & 1.0 & 0.3 & 2.8 \\
\hline -Other Sprains \& Strains & $840-845,848$ & 10 & 2.1 & 1.0 & 4.2 \\
\hline -Open Wounds - Head, Neck, Trunk & $870-879$ & 4 & 0.9 & 0.3 & 3.2 \\
\hline -Open Wounds - Upper Limb & $880-887$ & 3 & 0.8 & 0.2 & 3.2 \\
\hline -Open Wounds - Lower Limb & $890-897$ & 2 & 0.5 & 0.1 & 2.6 \\
\hline -Superficial Injuries & $910-919$ & 3 & 0.6 & 0.2 & 1.9 \\
\hline -Bruises & $920-924$ & 5 & 1.3 & 0.5 & 3.5 \\
\hline -Foreign Bodies Entering Orifice & $930-939$ & 3 & 0.8 & 0.2 & 2.7 \\
\hline -Burns & $940-949$ & 1 & 0.4 & 0.1 & 2.9 \\
\hline $\begin{array}{l}\text {-Adverse Reactions to Nonmedical } \\
\text { Substances or External Causes }\end{array}$ & $980-995$ & 1 & 0.2 & 0.0 & 1.4 \\
\hline -All Other Injuries & $\begin{array}{r}850-854,860-869,900-909 \\
925-929,950-979,996-999\end{array}$ & 20 & 4.4 & 2.7 & 7.3 \\
\hline Health Status/Health Service Contact & V01-V82 & 0 & 0 & 0 & 0 \\
\hline Total & & 123 & 30.4 & 24.9 & 37.2 \\
\hline
\end{tabular}

*Standardized to age distribution of 2000 U.S. population. 